\title{
DIAGRAM ALGEBRAS, HECKE ALGEBRAS AND DECOMPOSITION NUMBERS AT ROOTS OF UNITY
}

\author{
BY J.J. GRAHAM AND G.I. LEHRER
}

AbStract. - We prove that the cell modules of the affine Temperley-Lieb algebra have the same composition factors, when regarded as modules for the affine Hecke algebra of type $A$, as certain standard modules which are defined homologically. En route, we relate these to the cell modules of the Temperley-Lieb algebra of type $B$, which provides a connection between Temperley-Lieb algebras on $n$ and $n-1$ strings. Applications include the explicit determination of some decomposition numbers of standard modules at roots of unity, which in turn has implications for certain Kazhdan-Lusztig polynomials associated with nilpotent orbit closures. The methods involve the study of the relationships among several algebras defined by concatenation of braid-like diagrams and between these and Hecke algebras. Connections are made with earlier work of Bernstein-Zelevinsky on the "generic case" and of Jones on link invariants.

(C) 2003 Éditions scientifiques et médicales Elsevier SAS

RÉSUMÉ. - Nous démontrons que les "modules cellulaires" de l'algèbre de Temperley-Lieb affine ont, regardés comme modules pour l'algèbre de Hecke affine de type $A$, les mêmes facteurs de composition que certains modules "standards" qui sont définis homologiquement. Au passage, nous relions ces modules aux modules cellulaires pour l'algèbre de Temperley-Lieb de type $B$. Parmi les applications est la détermination explicite des nombres de décomposition de certains modules standards aux racines de l'unité, qui implique à son tour la détermination de certains polynômes de Kazhdan-Lusztig associés aux clôtures d'orbites nilpotentes. Nos méthodes consistent à étudier les rapports entre certaines algèbres de concaténation de diagrammes de tresses ou analogues, et entre ces algèbres et les algèbres de Hecke. Le travail est aussi relié aux travaux précédents de Bernstein-Zelevinski dans le cas "génerique" et de Jones sur les invariants des entrelacs.

(C) 2003 Éditions scientifiques et médicales Elsevier SAS

\section{Introduction}

Let $R$ be a commutative ring with 1 and let $q \in R^{\times}$, where for any ring $A$ with $1, A^{\times}$denotes the group of its invertible elements. In [14], we defined a "Temperley-Lieb category" $\mathbb{T}^{a}$, whose objects are the natural numbers $\mathbb{N}$, and whose morphisms are $R$-linear combinations of "planar diagrams from $t$ to $n$ " (for $t, n \in \mathbb{N}$ ), with composition depending on the element $q$. In particular, we have the algebras of endomorphisms

$$
T_{n}^{a}(q):=\operatorname{Hom}_{\mathbb{T}^{a}}(n, n), \quad \text { for } n=0,1,2,3, \ldots
$$

These were called in [14] the affine Temperley-Lieb algebras. Using a calculus of diagrams, together with the philosophy of cellular algebras, we developed in [op. cit.] a theory of cell 
modules $W_{t, z}(n)\left(0 \leqslant t \leqslant n, n-t \in 2 \mathbb{Z}, z \in R^{\times}\right)$for the algebras $T_{n}^{a}(q)$, and gave a complete description of the composition factors of the cell modules, valid for all $q$, even for $q$ a root of unity, the most complicated case. The description of the irreducible $T_{n}^{a}(q)$-modules is valid when $R$ is any algebraically closed field. The analysis of the composition factors of the cell modules applies when $R$ is an algebraically closed field whose characteristic is either 0 or $p>0$ such that $p e>n$, where $e$ is the multiplicative order of $q^{2}$.

Now let $\widehat{H_{n}^{a}}(q)$ be the extended affine Hecke algebra of type $\widetilde{A_{n-1}}$, which corresponds to $G=G L_{n}(\mathbb{C})$. This is the algebra considered in $[4,28,19]$, for which there is a theory of "standard modules" $M_{s, N}$, which may be constructed by regarding $\widehat{H_{n}^{a}}(q)$ as a convolution algebra of coherent sheaves acting on the Borel-Moore homology of certain varieties $\mathcal{V}_{s, N}$, where $s$ is a semisimple element of $G$ and $N$ is a nilpotent element of $\mathfrak{G}=\operatorname{Lie}(G)$ such that $\operatorname{Ad}(s) N=q^{2} N$. When $q$ is not a root of unity, the structure of the modules $M_{s, N}$ is fairly well understood, while when $q$ is a root of unity, it was conjectured in [20] and proved in [1] (cf. also [10]), that the decomposition numbers of the standard modules, i.e. the multiplicities of the irreducibles in $M_{s, N}$, are given by values of certain Kazhdan-Lusztig polynomials, which are generally not known explicitly (see also [21]).

In this work, we show that the cell modules $W_{t, z}(n)$ of the algebra $T_{n}^{a}(q)$ (cf. [14]) may be inflated via a family $\left\{\psi_{\alpha} \mid \alpha \in R^{\times}\right\}$of surjections $\widehat{H_{n}^{a}}(q) \rightarrow T_{n}^{a}(q)$ (see (5.12) below) to modules for $\widehat{H_{n}^{a}}(q)$, which we identify explicitly (in the Grothendieck group $\Gamma\left(\widehat{H_{n}^{a}}(q)\right)$ of finite dimensional $\widehat{H_{n}^{a}}(q)$-modules) with the standard modules $M_{s, N}$ where $N$ has just two Jordan blocks. This enables us to use the results of [14] to give completely explicit decompositions of these standard modules, and therefore give character formulae for their irreducible heads. We also obtain much detailed information about their internal structure. Among the consequences of our results are the statements that $M_{s, N}$ is always multiplicity free, and that when $q$ is a root of unity, the composition length may be arbitrarily large (as $n$ increases). The key point in this work is the identification of the inflations of our cell modules with the standard modules up to Grothendieck equivalence (Theorem (9.8)).

To achieve this, we show that the inflations $\psi_{\alpha}^{*} W_{t, z}(n)$ are generically (i.e. for generic $q$ ) induced modules from a parabolic subalgebra of $\widehat{H_{n}^{a}}(q)$. For this we need to understand the action of the "translation elements" $X_{i}$ on the inflations. This in turn depends on the relationship between two (known) ways of viewing $\widehat{H_{n}^{a}}(q)$; the first as a twisted tensor product of the group ring $R[\langle V\rangle]$ of $\mathbb{Z} \cong\langle V\rangle$ with the Hecke algebra $H_{n}^{a}(q)$ of the Coxeter system of affine type $\widetilde{A_{n-1}}$, the second as the tensor product of the finite dimensional Hecke algebra $H_{n}(q)$ of type $A_{n-1}$ with the $R$-algebra of Laurent polynomials $R\left[X_{1}^{ \pm 1}, \ldots, X_{n}^{ \pm 1}\right]$. We approach this relationship via generalised Artin braid groups. In addition, we shall have recourse to the "Temperley-Lieb algebra" of type $B_{n}$, denoted by $T L B_{n}(q, Q)$ below (it is sometimes referred to as the "blob algebra"), to determine the action of the $X_{i}$, since $T_{n-1}^{a}(q)$ is not naturally a subalgebra of $T_{n}^{a}(q)$, and therefore one does not have restriction. We circumvent this difficulty by proving that for any $Q$, there is a pair of natural surjections from $T_{n}^{a}(q)$ to $T L B_{n}(q, Q)$, and studying restriction from $T L B_{n}(q, Q)$ to $T L B_{n-1}(q, Q)$. This could be used to study the "modular representation theory" of $\widehat{H_{n}^{a}}(q)$, but we do not do this here. In the final Section 11 we interpret our results in terms of a generalisation to the non-generic case of the "multisegments" of Zelevinsky and Bernstein.

Since the "annular algebras" of V. Jones [16] are quotients of the algebras $T_{n}^{a}(q)$, their representation theory may be thought of as a subset of the story below. Hence our work throws light on the connection between the work of Jones on link invariants (cf. [17]) and affine Hecke algebras. 


\section{Some generalised Artin braid groups}

Let $W$ be the symmetric group $\operatorname{Sym}_{n}$, realised as a Coxeter group generated by the reflections $s_{i}$ in the hyperplanes $z_{i}-z_{i+1}=0$ of $V=\mathbb{C}^{n}(i=1,2, \ldots, n-1)$. Write $s_{n}$ for reflection in the affine hyperplane $z_{1}-z_{n}=1$. Then $\left\{s_{1}, \ldots, s_{n}\right\}$ are Coxeter generators for the affine Weyl group $W^{a} \cong W \ltimes \mathbb{Z}^{n-1}$, which may also be thought of as generated by $W$ together with translations by vectors $\left(a_{1}, \ldots, a_{n}\right)$, with $a_{i} \in \mathbb{Z}$ and $a_{1}+\cdots+a_{n}=0$. Write $\widehat{W^{a}}$ for the semidirect product of $W$ with the group $\mathbb{Z}^{n}$ of all translations by vectors with integer coordinates. Write $v_{0}=(1,1, \ldots, 1)$ and denote by $p_{0}$ the orthogonal projection $p_{0}: V \rightarrow v_{0}^{\perp}$. Then $W$ and $W^{a}$ act irreducibly as Coxeter groups on $V_{0}=v_{0}^{\perp}$, and $W^{a}$ is a normal subgroup of $\widehat{W^{a}}$, with quotient $\mathbb{Z}$.

The reflecting hyperplanes of $W^{a}$ acting on $V$ are the hyperplanes $z_{i}-z_{j}=k$, for $1 \leqslant i<j \leqslant n$ and $k \in \mathbb{Z}$. Write $M^{a}$ for the complement of these hyperplanes in $V$ and $M_{0}^{a}$ for $p_{0}\left(M^{a}\right)$. Thus, explicitly,

$$
M^{a}=\left\{\left(x_{1}, x_{2}, \ldots, x_{n}\right) \in V=\mathbb{C}^{n} \mid x_{i}-x_{j} \notin \mathbb{Z} \text { if } i \neq j\right\} .
$$

(2.1) Proposition (Nguyen [27]). - The fundamental group $\pi_{1}\left(M_{0}^{a} / W^{a}\right)$ is isomorphic to the Artin group associated to the Coxeter system $\left(W^{a},\left\{s_{1}, \ldots, s_{n}\right\}\right)$.

In fact Nguyen proves this for any affine type Coxeter group by giving a "cell decomposition" of the space $M_{0}^{a} / W^{a}$. In our case we have explicitly that $\pi_{1}\left(M_{0}^{a} / W^{a}\right)$, which we denote henceforth by $\Delta_{n}$, is generated by elements $\left\{\sigma_{1}, \sigma_{2}, \ldots, \sigma_{n}\right\}$ subject to the relations

$$
\begin{aligned}
\sigma_{i} \sigma_{j} & =\sigma_{j} \sigma_{i} \quad \text { if } j \not \equiv i \pm 1(\bmod n), \\
\sigma_{i} \sigma_{i+1} \sigma_{i} & =\sigma_{i+1} \sigma_{i} \sigma_{i+1} \quad \text { for } i=1,2, \ldots, n,
\end{aligned}
$$

where the subscripts in (BR) are taken $\bmod n$.

(2.2) LEMMA. - The map $p_{0}: M^{a} \rightarrow M_{0}^{a}$ is a $W^{a}$-homotopy equivalence. Hence the quotient spaces $M^{a} / W^{a}$ and $M_{0}^{a} / W^{a}$ are homotopy equivalent. In particular they have the same fundamental group.

Proof. - If $I$ is the unit interval and $i$ is the inclusion of $M_{0}^{a}$ in $M^{a}$, then $p_{0} \circ i=\operatorname{id}_{M_{0}^{a}}$, and the map $(v, t) \mapsto v-(1-t) \frac{\left\langle v, v_{0}\right\rangle}{n} v_{0}\left(M^{a} \times I \rightarrow M^{a}\right)$ defines a homotopy from $i \circ p_{0}$ to $\operatorname{id}_{M^{a}}$, which commutes with the $W^{a}$ action for each $t \in I$.

(2.3) LEMMA. - Let $\widehat{W^{a}} \supset W^{a}$ be as described above. The map $M^{a} / W^{a} \rightarrow M^{a} / \widehat{W^{a}}$ is an unramified covering with covering group $\mathbb{Z}$.

The proof is easy. Note that the quotient $\widehat{W^{a}} / W^{a}$ is generated by the element

$$
\tau^{\prime}=\left(c_{n},(1,0,0, \ldots, 0)\right) \in \operatorname{Sym}_{n} \ltimes \mathbb{Z}^{n},
$$

where $c_{n}$ is the $n$-cycle $(12 \ldots n) \in \operatorname{Sym}_{n}$. This element has the property that $\tau^{\prime n}$ lies in the centre $Z\left(\widehat{W^{a}}\right)$. It follows from the lemma that $M^{a} / \widehat{W^{a}}$ may be thought of as the quotient of $M^{a} / W^{a}$ by the cyclic group $\left\langle\tau^{\prime}\right\rangle$.

(2.4) COROLLARY. - There is an exact sequence

$$
1 \rightarrow \pi_{1}\left(M^{a} / W^{a}\right) \rightarrow \pi_{1}\left(M^{a} / \widehat{W^{a}}\right) \rightarrow \mathbb{Z} \rightarrow 1 .
$$


We now identify the space $M^{a} / \widehat{W^{a}}$ and its fundamental group. For any topological space $Y$, let $X_{n}(Y)$ be the space

$$
X_{n}(Y)=\left\{\left(y_{1}, \ldots, y_{n}\right) \in Y^{n} \mid y_{i} \neq y_{j} \text { if } i \neq j\right\} / \operatorname{Sym}_{n} .
$$

Then evidently $M^{a} / \widehat{W^{a}} \cong X_{n}(\mathbb{C} / \mathbb{Z}) \cong X_{n}\left(\mathbb{C}^{*}\right)$. But if $M_{B_{n}}$ is the complex hyperplane complement of type $B_{n}$ (viz. $\mathbb{C}^{n}$ with the hyperplanes $x_{i} \pm x_{j}=0$ and $x_{i}=0$ removed), and $W_{B_{n}}$ is the corresponding Weyl group, then clearly

$$
M_{B_{n}} / W_{B_{n}} \cong X_{n}\left(\mathbb{C}^{*}\right) /\{ \pm \mathrm{id}\} \cong X_{n}\left(\mathbb{C}^{*}\right) \cong M_{0}^{a} / \widehat{W^{a}} .
$$

But Deligne has shown [7] that the space of regular orbits of a finite Coxeter group on its complexified reflection representation space is its associated generalised Artin braid group (this is also proved in [27]). Write $\Gamma_{n}$ for the generalised Artin braid group of type $B_{n}$. Then $\Gamma_{n}$ has generators $\left\{\xi_{1}, \sigma_{1}, \sigma_{2}, \ldots, \sigma_{n-1}\right\}$, with relations

$$
\begin{aligned}
\sigma_{i} \sigma_{j} & =\sigma_{j} \sigma_{i} \quad \text { if }|i-j| \neq 1, \\
\sigma_{i} \sigma_{i+1} \sigma_{i} & =\sigma_{i+1} \sigma_{i} \sigma_{i+1} \quad \text { for } i=1,2, \ldots, n-2, \\
\xi_{1} \sigma_{1} \xi_{1} \sigma_{1} & =\sigma_{1} \xi_{1} \sigma_{1} \xi_{1} \\
\xi_{1} \sigma_{i} & =\sigma_{i} \xi_{1} \quad \text { if } i \neq 1 .
\end{aligned}
$$

Now paths in $X_{n}\left(\mathbb{C}^{*}\right)$ may be regarded as periodic braids, or braids on a thickened cylinder, as follows. Think of $\mathbb{C}^{*}$ as the plane with a large hole at 0 ; choose $n$ points $P_{1}, P_{2}, \ldots, P_{n}$ in $\mathbb{C}^{*}$. A path in $X_{n}\left(\mathbb{C}^{*}\right)$ may then be regarded as a braid "around the hole", or on the thickened cylinder, where each string starts at some $P_{i}$ and finishes at $P_{i^{\prime}}$, where $i \mapsto i^{\prime}$ is a permutation of $\{1,2, \ldots, n\}$. If we cut the cylinder open, these braids may be drawn in the plane, and regarded as "periodic braids", or cylindrical braids. These may be drawn as depicted in the diagrams in Fig. 1, in which the two intervals labelled $A B$ are identified by bending the rectangle in towards the page.

Now let $\tau \in \Gamma_{n}$ be the "twist" as shown (it corresponds to the projection of a path in $M_{0}^{a} / W^{a}$ from the base point $P$ to $\tau^{\prime} P$, where $\tau^{\prime}$ is the element defined above, such that $\left.M^{a} / \widehat{W^{a}} \cong\left(M^{a} / W^{a}\right) /\left\langle\tau^{\prime}\right\rangle\right)$. Further, let $\sigma_{i}$ be the generating braids depicted in the diagram $(i=1,2, \ldots, n)$. It is clear that $\Gamma_{n}$ then has a presentation with generators $\left\{\tau, \sigma_{1}, \sigma_{2}, \ldots, \sigma_{n}\right\}$, with relations (BR) for the $\sigma_{i}$, together with

$$
\tau \sigma_{i} \tau^{-1}=\sigma_{i+1} \quad \text { for } i=1,2, \ldots, n,
$$

where the subscripts are taken $\bmod n$.

(2.6) Proposition. - With the above notation, let

$$
\begin{aligned}
\xi_{1} & =\tau \sigma_{n-1}^{-1} \sigma_{n-2}^{-1} \ldots \sigma_{1}^{-1} \quad \text { and } \\
\xi_{i+1} & =\sigma_{i} \xi_{i} \sigma_{i} \quad \text { for } i=1,2, \ldots, n-1 .
\end{aligned}
$$

Then the family $\left\{\xi_{1}, \sigma_{1}, \ldots, \sigma_{n-1}\right\}$ generates $\Gamma_{n}$ subject to the relations (BRB), and $\left\{\xi_{1}, \ldots, \xi_{n}\right\}$ generates a free abelian group of rank $n$. 


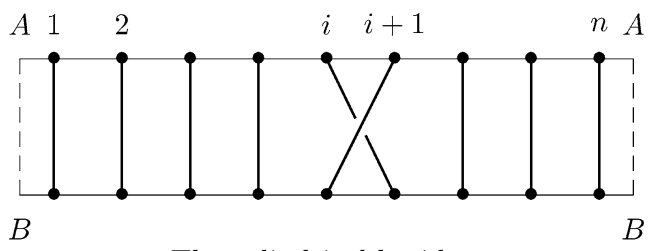

The cylindrical braid $\sigma_{i}$

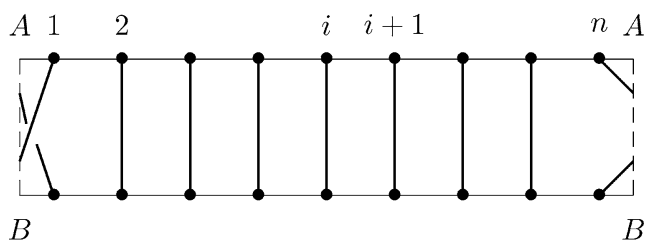

The cylindrical braid $\sigma_{n}$

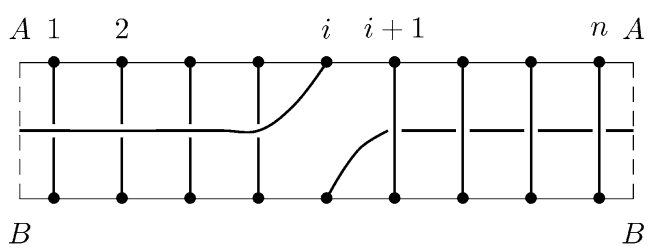

The cylindrical braid $\xi_{i}$

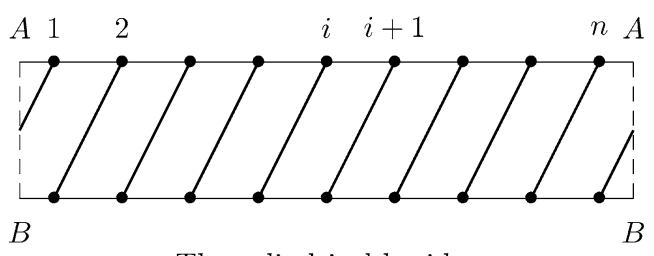

The cylindrical braid $\tau$

Fig. 1.

Proof. - It is clear that $\left\{\xi_{1}, \sigma_{1}, \ldots, \sigma_{n-1}\right\}$ generates $\Gamma_{n}$, since $\left\{\tau, \sigma_{1}, \sigma_{2}, \ldots, \sigma_{n-1}\right\}$ does, and $\tau=\xi_{1} \sigma_{1} \ldots \sigma_{n-1}$. We next show that $\left\{\xi_{1}, \sigma_{1}, \ldots, \sigma_{n-1}\right\}$ satisfies the relations (BRB). This is easy to see from the braid pictures (see Fig. 1), but we shall provide an algebraic proof. For $i=1,2, \ldots, n$, we have

$$
\xi_{i}=\sigma_{i-1} \sigma_{i-2} \ldots \sigma_{1} \tau \sigma_{n-1}^{-1} \sigma_{n-2}^{-1} \ldots \sigma_{i}^{-1}
$$

We shall first show that

$$
\sigma_{j} \xi_{i}=\xi_{i} \sigma_{j} \text { if } j \neq i-1, i \text {. }
$$

To see (2.6.1), first take $i=1$. Then for $j>1$,

$$
\begin{aligned}
\sigma_{j} \xi_{1} & =\sigma_{j} \tau \sigma_{n-1}^{-1} \sigma_{n-2}^{-1} \ldots \sigma_{1}^{-1}=\tau \sigma_{j-1}^{-1} \sigma_{n-1}^{-1} \ldots \sigma_{1}^{-1} \\
& =\tau \sigma_{n-1}^{-1} \ldots \sigma_{j+1}^{-1} \sigma_{j-1} \sigma_{j}^{-1} \sigma_{j-1}^{-1} \sigma_{j-2}^{-1} \ldots \sigma_{1}^{-1} \\
& =\tau \sigma_{n-1}^{-1} \ldots \sigma_{j+1}^{-1} \sigma_{j}^{-1} \sigma_{j-1}^{-1} \sigma_{j} \sigma_{j-2}^{-1} \ldots \sigma_{1}^{-1} \text { by (BR) } \\
& =\xi_{1} \sigma_{j} .
\end{aligned}
$$


This proves (2.6.1) for $i=1$. But since $\xi_{i}=\sigma_{i-1} \sigma_{i-2} \ldots \sigma_{1} \xi_{1} \sigma_{1} \ldots \sigma_{i-1}$, it follows that (2.6.1) holds for all $j>i$. Now take $j<i-1$. Then

$$
\begin{aligned}
\sigma_{j} \xi_{i} & =\sigma_{j} \sigma_{i-1} \sigma_{i-2} \ldots \sigma_{1} \tau \sigma_{n-1}^{-1} \ldots \sigma_{i}^{-1} \\
& =\sigma_{i-1} \ldots \sigma_{j} \sigma_{j+1} \sigma_{j} \sigma_{j-1} \ldots \sigma_{1} \tau \sigma_{n-1}^{-1} \ldots \sigma_{i}^{-1} \\
& =\sigma_{i-1} \ldots \sigma_{j+1} \sigma_{j} \sigma_{j+1} \sigma_{j-1} \ldots \sigma_{1} \tau \sigma_{n-1}^{-1} \ldots \sigma_{i}^{-1} \quad \text { by (BR) } \\
& =\xi_{i} \sigma_{j} .
\end{aligned}
$$

This proves (2.6.1). We next show that

$$
\xi_{1} \sigma_{1} \xi_{1} \sigma_{1}=\sigma_{1} \xi_{1} \sigma_{1} \xi_{1}
$$

Let

$$
\beta_{n}=\sigma_{n-1} \sigma_{n-2}^{-1} \ldots \sigma_{1}^{-1} \sigma_{n-1}^{-1} \sigma_{n-2}^{-1} \ldots \sigma_{1}^{-1} .
$$

We shall show by induction on $n$ that

$$
\beta_{n}=\sigma_{n-2}^{-1} \sigma_{n-1}^{-1} \sigma_{n-3}^{-1} \sigma_{n-2}^{-1} \ldots \sigma_{1}^{-1} \sigma_{2}^{-1} .
$$

If $n=2$, both sides of (2.6.2.1) are equal to 1. In general,

$$
\begin{aligned}
\beta_{n} & =\sigma_{n-1} \sigma_{n-2}^{-1} \sigma_{n-1}^{-1} \sigma_{n-3}^{-1} \ldots \sigma_{1}^{-1} \sigma_{n-2}^{-1} \sigma_{n-3}^{-1} \ldots \sigma_{1}^{-1} \\
& =\sigma_{n-2}^{-1} \sigma_{n-1}^{-1} \sigma_{n-2} \sigma_{n-3}^{-1} \ldots \sigma_{1}^{-1} \sigma_{n-1}^{-1} \sigma_{n-2}^{-1} \ldots \sigma_{1}^{-1} \\
& =\sigma_{n-2}^{-1} \sigma_{n-1}^{-1} \beta_{n-1},
\end{aligned}
$$

which proves (2.6.2.1).

Next, observe that if $\gamma_{n}=\sigma_{n-2}^{-1} \ldots \sigma_{1}^{-1} \sigma_{n-1}^{-1} \sigma_{n-2}^{-1} \ldots \sigma_{2}^{-1}$, then $\gamma_{n}=\beta_{n}$. For $n=3$, $\gamma_{n}=\beta_{n}=\sigma_{1}^{-1} \sigma_{2}^{-1}$. In general, $\gamma_{n}=\sigma_{n-2}^{-1} \sigma_{n-1}^{-1} \gamma_{n-1}$, and so

$$
\gamma_{n}=\beta_{n}
$$

for all $n$, by induction on $n$.

To prove (2.6.2), we now have

$$
\begin{aligned}
\sigma_{1} \xi_{1} \sigma_{1} \xi_{1} & =\sigma_{1} \tau \sigma_{n-1}^{-1} \sigma_{n-2}^{-1} \ldots \sigma_{2}^{-1} \tau \sigma_{n-1}^{-1} \sigma_{n-2}^{-1} \ldots \sigma_{1}^{-1} \\
& =\sigma_{1} \tau^{2} \sigma_{n-2}^{-1} \sigma_{n-3}^{-1} \ldots \sigma_{1}^{-1} \sigma_{n-1}^{-1} \sigma_{n-2}^{-1} \sigma_{n-3}^{-1} \ldots \sigma_{1}^{-1} \\
& =\tau^{2} \sigma_{n-1} \sigma_{n-2}^{-1} \sigma_{n-3}^{-1} \ldots \sigma_{1}^{-1} \sigma_{n-1}^{-1} \sigma_{n-2}^{-1} \ldots \sigma_{1}^{-1} \\
& =\tau^{2} \beta_{n} .
\end{aligned}
$$

But

$$
\begin{aligned}
\xi_{1} \sigma_{1} \xi_{1} \sigma_{1} & =\tau \sigma_{n-1}^{-1} \sigma_{n-2}^{-1} \ldots \sigma_{2}^{-1} \tau \sigma_{n-1}^{-1} \sigma_{n-2}^{-1} \ldots \sigma_{2}^{-1} \\
& =\tau^{2} \sigma_{n-2}^{-1} \sigma_{n-3}^{-1} \ldots \sigma_{1}^{-1} \sigma_{n-1}^{-1} \sigma_{n-2}^{-1} \ldots \sigma_{2}^{-1} \\
& =\tau^{2} \gamma_{n} \\
& =\tau^{2} \beta_{n} \quad \text { by }(2.6 .2 .2) \\
& =\sigma_{1} \xi_{1} \sigma_{1} \xi_{1},
\end{aligned}
$$

which proves (2.6.2). 
It follows immediately from (2.6.2) and (2.6.1) that the family $\left\{\xi_{1}, \sigma_{1}, \ldots, \sigma_{n-1}\right\}$ satisfies the relations (BRB). But the relations (BR) and $\tau \sigma_{i} \tau^{-1}=\sigma_{i+1}$ are similarly shown to follow from (BRB), whence we have a presentation of $\Gamma_{n}$.

It remains to show that the $\xi_{i}$ commute with each other. Note that (2.6.2) says that $\xi_{1} \xi_{2}=\xi_{2} \xi_{1}$. In general, suppose that we know $\xi_{i} \xi_{j}=\xi_{j} \xi_{i}$ for all $j>i, 1 \leqslant i<k$. Since $\xi_{k}=\sigma_{k-1} \xi_{k-1} \sigma_{k-1}$, if $j>k, \xi_{j}$ commutes with $\sigma_{k-1}$ by (2.6.1), and with $\xi_{k-1}$ by induction, and hence with $\xi_{k}$. Hence by induction the $\xi_{i}$ all commute. To see that there are no relations among the $\xi_{i}$, one may use the braid picture as follows. In any cylindrical braid of the form $\xi_{1}^{m_{1}} \ldots \xi_{n}^{m_{n}}$, note that each string joins points on the top and bottom of the cylinder which have the same label, since this is true of each $\xi_{i}$. Given the braid, the indices $m_{i}$ may be recovered as the number of times the relevant string winds around the cylinder.

(2.7) COROLlARY. - The exact sequence (2.4) is realised as

$$
1 \rightarrow \Delta_{n} \rightarrow \Gamma_{n} \rightarrow \mathbb{Z} \rightarrow 1
$$

where $\Delta_{n} \rightarrow \Gamma_{n}$ is inclusion and $\Gamma_{n} \rightarrow \mathbb{Z}$ is the map taking $\tau^{r} \sigma_{i_{1}}^{n_{1}} \ldots \sigma_{i_{l}}^{n_{l}} \in \Gamma_{n}$ to $r \in \mathbb{Z}$.

Our final result in this section is

(2.8) LEMMA. - We have the following relation in $\Gamma_{n}$.

$$
\tau^{n}=\xi_{1} \xi_{2} \ldots \xi_{n}
$$

Proof. - Since $\xi_{1}=\tau \sigma_{n-1}^{-1} \sigma_{n-2}^{-1} \ldots \sigma_{1}^{-1}$ and $\xi_{i+1}=\sigma_{i} \xi_{i} \sigma_{i}$, one shows easily by induction on $i$ that for $1 \leqslant i \leqslant n-1$,

$$
\xi_{1} \xi_{2} \ldots \xi_{i}=\tau^{i} \sigma_{n-i}^{-1} \ldots \sigma_{1}^{-1} \sigma_{n-i+1}^{-1} \ldots \sigma_{2}^{-1} \ldots \sigma_{n-1}^{-1} \ldots \sigma_{i}^{-1} .
$$

Hence $\xi_{1} \xi_{2} \ldots \xi_{n-1}=\tau^{n-1} \sigma_{1}^{-1} \ldots \sigma_{n-1}^{-1}=\tau^{n} \xi_{n}^{-1}$.

\section{Affine Hecke algebras of type $A$}

Let $R$ be a commutative ring, denote by $R^{\times}$the group of invertible elements of $R$, and let $q \in R^{\times}$. We maintain the notation of the last section, so that $\Gamma_{n}$ is the Artin braid group $B\left(B_{n}\right)$ of type $B_{n}$ and $\Delta_{n}$ is the Artin braid group $B\left(\widetilde{A_{n-1}}\right)$ of type $\widetilde{A_{n-1}}$, regarded as the subgroup of $\Gamma_{n}$ generated by $\sigma_{1}, \ldots, \sigma_{n}$. Denote by $R \Gamma_{n}$ the group ring of $\Gamma_{n}$ over $R$.

(3.1) Definition. - Let $S_{i}$ be the element $S_{i}=\left(\sigma_{i}-q\right)\left(\sigma_{i}+q^{-1}\right)$ of $R \Gamma_{n}(i=1,2, \ldots, n)$. The affine Hecke algebra $\widehat{H_{n}^{a}}(q)$ of $G L_{n}$ over $R$ is defined by

$$
\widehat{H_{n}^{a}}(q)=R \Gamma_{n} /\left\langle S_{1}\right\rangle .
$$

Note that since $S_{1}, \ldots, S_{n}$ are all conjugate in $\mathbb{R} \Gamma_{n}$, the ideal $\left\langle S_{1}\right\rangle$ is equal to $\left\langle S_{1}, \ldots, S_{n}\right\rangle$. Let $\eta: R \Gamma_{n} \rightarrow \widehat{H_{n}^{a}}(q)$ be the natural map. We then write

$$
\begin{gathered}
\eta\left(\sigma_{i}\right)=T_{i} \quad \text { for } i=1, \ldots, n, \\
\eta\left(\xi_{i}\right)=X_{i} \quad \text { for } i=1, \ldots, n, \\
\eta(\tau)=V
\end{gathered}
$$


The next proposition collects some well known facts concerning $\widehat{H_{n}^{a}}(q)$, many of which may be found in $\S 3$ of [24].

(3.3) Proposition. -

(i) The elements $T_{1}, \ldots, T_{n}$ generate a subalgebra $H_{n}^{a}(q)$ of $\widehat{H_{n}^{a}}(q)$, which has $R$-basis $\left\{T_{w} \mid w \in W^{a} \cong \operatorname{Sym}_{n} \ltimes \mathbb{Z}^{n-1}\right\}$, where, if $w=s_{i_{1}} \ldots s_{i_{\ell}}$ is a reduced expression for $w \in W^{a}$, $T_{w}=T_{i_{1}} \ldots T_{i_{\ell}}$. We refer to this as the "unextended" Hecke algebra of type $\widetilde{A_{n-1}}$.

(ii) The elements $T_{1}, \ldots, T_{n-1}$ generate a subalgebra $H_{n}(q)$ of $\widehat{H_{n}^{a}}(q)$ which has (finite) $R$-basis $\left\{T_{w} \mid w \in W \cong \operatorname{Sym}_{n}\right\}$.

(iii) We have $\widehat{H_{n}^{a}}(q) \cong R \mathbb{Z} \otimes H_{n}^{a}(q) \cong R\langle V\rangle \otimes H_{n}^{a}(q)$, where the tensor product is twisted, using the action of $V$ on $H_{n}^{a}(q): V T_{i} V^{-1}=T_{i+1}$, where the subscript is taken $\bmod n$.

(iv) We have $\widehat{H_{n}^{a}}(q) \cong H_{n}(q) \otimes R\left[X_{1}^{ \pm 1}, \ldots, X_{n}^{ \pm 1}\right]$ as $R$-module, and the multiplication is given by the "Bernstein relations": for $i \in\{1, \ldots, n-1\}$, write $s_{i}$ for the corresponding simple reflection in $W$ and ${ }^{s} f$ for the image of $f \in R\left[X_{1}^{ \pm 1}, \ldots, X_{n}^{ \pm 1}\right]$ under the natural action of $W \cong \operatorname{Sym}_{n}$. Then

$$
T_{i} f-\left({ }^{s_{i}} f\right) T_{i}=\left(q-q^{-1}\right) \frac{f-\left({ }^{s_{i}} f\right)}{1-X_{i} X_{i+1}^{-1}} .
$$

(v) The centre $Z\left(\widehat{H_{n}^{a}}(q)\right)$ is the ring of symmetric functions in the $X_{i}^{ \pm 1}$. Equivalently, $Z\left(\widehat{H_{n}^{a}}(q)\right)=R\left[\Sigma_{1}^{ \pm 1}, \ldots, \Sigma_{n}^{ \pm 1}\right]$, where $\Sigma_{i}$ is the ith elementary symmetric function in the $X_{i}$.

Note that some authors use notation which results in the denominator of (iv) above being $1-X_{i+1} X_{i}^{-1}$.

We remark that to prove the relation in (iv) from those given in Section 2 for the braid group, one observes that the relation is linear in $f$, and hence need only be proved for monomials; moreover one easily shows that if the relation holds for $f_{1}$ and $f_{2}$, then it holds for $f_{1} f_{2}$. Thus one is reduced to proving the relation for $f=X_{j}$, which is easy.

In addition to the algebras above, we shall need to consider the (finite rank) Hecke algebra of type $B$, which arises as follows. Let $W B:=\operatorname{Sym}_{n} \ltimes(\mathbb{Z} / 2 \mathbb{Z})^{n}$ be the hyperoctahedral group. This is generated as Coxeter group by $\left\{s_{1}, \ldots, s_{n-1}\right\}$, together with another generator $s_{0}$. The generators $\left\{s_{0}, s_{1}, \ldots, s_{n-1}\right\}$ are involutions, and satisfy the relations analogous to (BRB) above. Let $Q \in R^{\times}$. The Hecke algebra $H B_{n}(q, Q)$ of type $B_{n}$ with parameters $(q, Q)$ is defined as

$$
\begin{aligned}
H B_{n}(q, Q) & =\widehat{H_{n}^{a}}(q) /\left\langle\left(X_{1}-Q\right)\left(X_{1}+Q^{-1}\right)\right\rangle \\
& =R \Gamma_{n} /\left\langle\left(\xi_{1}-Q\right)\left(\xi_{1}+Q^{-1}\right),\left(\sigma_{1}-q\right)\left(\sigma_{1}+q^{-1}\right)\right\rangle
\end{aligned}
$$

(3.4) Proposition. - Let

$$
\eta_{Q}: \widehat{H_{n}^{a}}(q) \rightarrow H B_{n}(q, Q)
$$

be the natural map. Write $T_{i} \in H B_{n}(q, Q)$ for the image of $T_{i} \in \widehat{H_{n}^{a}}(q)$ under $\eta_{Q}$ $(i=1, \ldots, n-1)$ (relying on the context to distinguish between them), and write $T_{0}=\eta_{Q}\left(X_{1}\right)$. Then $H B_{n}(q, Q)$ has $R$-basis $\left\{T_{w} \mid w \in W B\right\}$, where, if $w=s_{i_{1}} \ldots s_{i_{\ell}}$ is a reduced expression for $w \in W B, T_{w}=T_{i_{1}} \ldots T_{i_{\ell}}$. 
The relationship among the various algebras introduced so far is illustrated in the commutative diagram below.

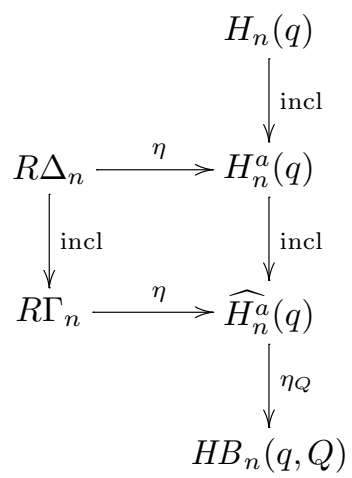

The relations discussed in Section 2 for the braid groups $\Gamma_{n}$ and $\Delta_{n}$ may be interpreted in the Hecke algebras as follows.

(3.6) LEMMA. - The following relations hold in $\widehat{H_{n}^{a}}(q)$.

$$
\begin{aligned}
V^{n} & =X_{1} X_{2} \ldots X_{n}, \\
V & =X_{1} T_{1} T_{2} \ldots T_{n-1} .
\end{aligned}
$$

If we write $V$ for $\eta_{Q}(V)$ and adopt the notation of (3.4), we also have (in $H B_{n}(q, Q)$ )

$$
V=T_{0} T_{1} \ldots T_{n-1}
$$

\section{Affine and finite dimensional Temperley-Lieb algebras}

Let $W=\left\langle s_{1}, \ldots, s_{n-1}\right\rangle \cong \operatorname{Sym}_{n}$ as above and write

$$
W_{i}=\left\langle s_{i}, s_{i+1}\right\rangle \cong \operatorname{Sym}_{3} \quad \text { for } i=1,2, \ldots, n-2 .
$$

Define the element $E_{i} \in H_{n}(q) \subset H_{n}^{a}(q) \subset \widehat{H_{n}^{a}}(q)$ by

$$
E_{i}=\sum_{w \in W_{i}} q^{\ell(w)} T_{w}
$$

where $\ell(w)$ denotes the usual length function. Let $I$ (resp. $\hat{I}$ ) denote the ideal of $H_{n}^{a}(q)$ (resp. $\left.\widehat{H_{n}^{a}}(q)\right)$ generated by $E_{1}$. Note that since the $E_{i}$ are all conjugate, this is the same as the ideal generated by all the $E_{i}$. by

(4.1) Definition. - The affine Temperley-Lieb algebras $T L_{n}^{a}(q)$ and $\widehat{T L_{n}^{a}}(q)$ are defined

$$
\begin{aligned}
& T L_{n}^{a}(q)=H_{n}^{a}(q) / I, \\
& \widehat{T L_{n}^{a}}(q)=\widehat{H_{n}^{a}}(q) / \hat{I} .
\end{aligned}
$$

It is known (cf. [14,15]) that if $C_{i}=-\left(T_{i}+q^{-1}\right) \in H_{n}^{a}(q)(i=1, \ldots, n)$, then in $H_{n}^{a}(q)$, $C_{i} C_{i+1} C_{i}-C_{i}=C_{i+1} C_{i} C_{i+1}-C_{i+1}=-q^{3} E_{i}$, where the indices are taken $\bmod n$. If we 
abuse notation by writing $C_{i} \in T L_{n}^{a}(q)$ for the image of $C_{i} \in H_{n}^{a}(q)$ under the natural map, it follows easily that $T L_{n}^{a}(q)$ is generated by $\left\{C_{1}, \ldots, C_{n}\right\}$ subject to the relations

$$
\begin{aligned}
C_{i}^{2} & =\delta_{q} C_{i}, \\
C_{i} C_{i \pm 1} C_{i} & =C_{i}, \\
C_{i} C_{j} & =C_{j} C_{i} \quad \text { if }|i-j| \geqslant 2 \text { and }\{i, j\} \neq\{1, n\},
\end{aligned}
$$

where, for any element $x \in R^{\times}, \delta_{x}:=-\left(x+x^{-1}\right)$.

Moreover it is easy to see that (cf. (3.3)(iii) above, or [15, §2])

$$
\widehat{T L_{n}^{a}}(q) \cong R\langle V\rangle \otimes T L_{n}^{a}(q)
$$

where $V$ permutes the $C_{i}$ cyclically.

Now in addition to the algebras $T L_{n}^{a}(q)$ and $\widehat{T L_{n}^{a}}(q)$, we shall require the algebra $T_{n}^{a}(q)$ which was defined in $[14,(2.7)]$ and referred to there (loc. cit.) as "the affine Temperley-Lieb algebra". This is defined as an algebra of diagrams or, more accurately, as the algebra of morphisms: $n \rightarrow n$ in the category $\mathbb{T}^{a}$ (see [14, (2.5)]) and $T L_{n}^{a}(q)$ is identified [14, (2.9)] as the subalgebra of $T_{n}^{a}(q)$ spanned by the "non-monic diagrams $: n \rightarrow n$ of even rank", together with the identity. It also occurs independently in the work of Green [11] and Fan-Green [8]. We shall need to make some use of the diagrammatic description in this work; details may be found in [14], but a good approximation to the picture is obtained if one thinks of affine diagrams as arcs drawn on the surface of a cylinder joining $2 n$ marked points, $n$ on each circle component of the boundary, in pairs. The arcs must not intersect, and diagrams are multiplied by concatenation in the usual way. These diagrams are represented by periodic diagrams drawn between two horizontal lines, each diagram being determined by the "fundamental rectangle", from which the cylinder is obtained by identifying vertical edges. In this interpretation, the generators $\left\{f_{1}, \ldots, f_{n}, \tau\right\}$ of $T_{n}^{a}(q)$ are represented by the diagrams in Fig. 2 . The elements $\left\{f_{1}, \ldots, f_{n}\right\}$ of the algebra $T_{n}^{a}(q)$ satisfy the relations (TL), with $C_{i}$ replaced by $f_{i}$, and it is noted in [14, (2.9)] that these generate an algebra isomorphic to $T L_{n}^{a}(q)$. Further, $\tau f_{i} \tau^{-1}=f_{i+1}$, where the index is taken $\bmod n$.
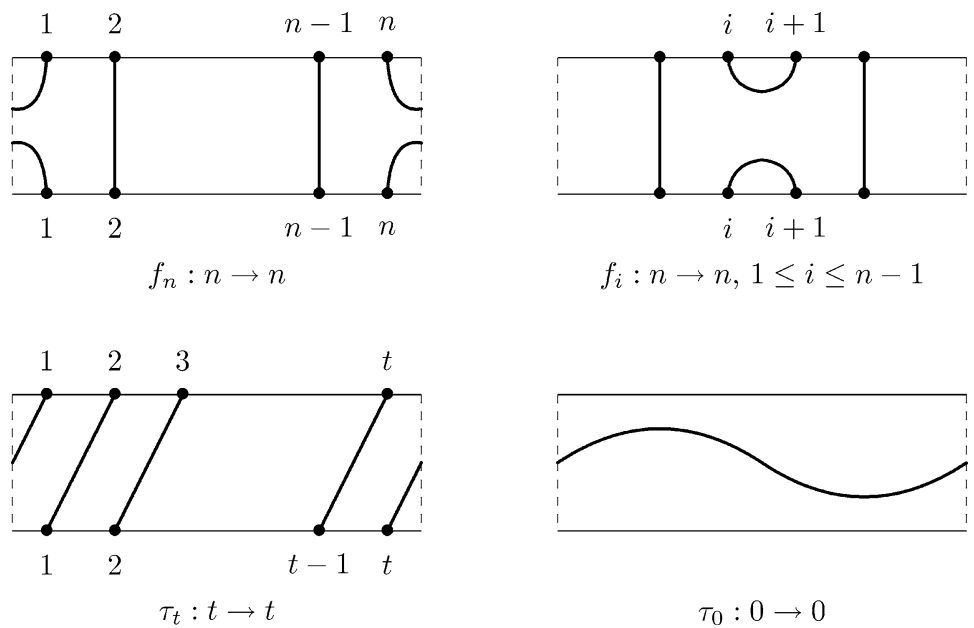

Fig. 2. 
(4.3) Proposition. -

(i) There is a family of surjections $\phi_{\alpha}: \widehat{T L_{n}^{a}}(q) \rightarrow T_{n}^{a}(q)\left(\alpha \in R^{\times}\right)$, defined by $\phi_{\alpha}\left(C_{i}\right)=f_{i}$ and $\phi_{\alpha}(V)=\alpha \tau$. Each $\phi_{\alpha}$ restricts to a monomorphism on $T L_{n}^{a}(q)$.

(ii) The kernel of $\phi_{\alpha}$ is generated by the element $\nu_{\alpha} \in \widehat{T L_{n}^{a}}(q)$, where

$$
\nu_{\alpha}=\alpha^{-2} V^{2} C_{n-1}-C_{1} C_{2} \ldots C_{n-1}=\alpha^{-2} C_{1} V^{2}-C_{1} C_{2} \ldots C_{n-1} .
$$

(iii) If $R$ is an algebraically closed field of characteristic prime to $n$, any irreducible finite dimensional representation of $\widehat{T L_{n}^{a}}(q)$ is the pullback via $\phi_{\alpha}$ (for some $\alpha \in R^{\times}$) of an irreducible representation of $T_{n}^{a}(q)$.

Proof. - The first part of (i) follows immediately from the relations above, while the second follows from the fact (cf. [14, 2.9]) that (TL) gives a presentation of $T L_{n}^{a}(q)$. Next, one verifies easily that $\tau^{2} f_{n-1}=f_{1} f_{2} \ldots f_{n-1}$ in $T_{n}^{a}(q)$ (see $[15,1.11]$ ), which shows that $\nu_{\alpha} \in \operatorname{Ker} \phi_{\alpha}$. The fact that $\nu_{\alpha}$ generates the kernel may be found in [11] or [8]. This relation also appears in [16]. The statement (iii) may be proved using the argument of Theorem 2.6 in [15].

In [14], we defined cell modules $W_{t, z}(n)$ for the algebra $T_{n}^{a}(q)$, (where $t \in \mathbb{Z}, 0 \leqslant t \leqslant n$, $t+n \in 2 \mathbb{Z}$, and $z \in R^{\times}$) and when $R$ is an algebraically closed field of characteristic zero, completely determined their composition factors and multiplicities. Our purpose here is to interpret these results for the pullbacks $\phi_{\alpha}^{*} W_{t, z}(n)$. To identify these pullbacks as standard modules for $\widehat{H_{n}^{a}}(q)$ up to Grothendieck equivalence (cf. [19] or [31]), we need to determine the action of the translation elements $X_{i}$ on the modules, and for this we shall require the TemperleyLieb algebra $T L B_{n}(q, Q)$ of type $B_{n}$.

\section{The Temperley-Lieb algebras of type $B$}

This algebra has been studied by mathematical physicists [25,26], where it is referred to as the "blob algebra", and in [29] (see also [6], and the references there). We shall present here the main facts which we require concerning $T L B_{n}(q, Q)$, relying for general background on op. cit. Our notation continues from Section 3 above, and we start with an algebraic definition of $T L B_{n}(q, Q)$.

Note first that the Hecke algebra $H B_{n}(q, Q)$ has an $R$-algebra homomorphism

$$
\varepsilon: H B_{n}(q, Q) \rightarrow R
$$

defined on the generators $T_{i}(i=0,1, \ldots, n-1$, see (3.4)) by

$$
\varepsilon\left(T_{0}\right)=Q, \quad \varepsilon\left(T_{i}\right)=q \text { for } 1 \leqslant i \leqslant n-1 .
$$

As above, let $s_{0}, s_{1}, \ldots, s_{n-1}$ be the simple generators of the hyperoctahedral group $W B$, and write $W_{i}=\left\langle s_{i}, s_{i+1}\right\rangle$ for $i=0,1, \ldots, n-2$. Then define $E_{i}=\sum_{w \in W_{i}} \varepsilon\left(T_{w}\right) T_{w}$. For $i \neq 0$, these $E_{i}$ coincide with the $E_{i}$ of Section 4, and they are all conjugate in $H B_{n}(q, Q)$. For $i>0$, let $C_{i}=-\left(T_{i}+q^{-1}\right)$, and let $C_{0}=-\left(T_{0}+Q^{-1}\right)$. The next lemma summarises several relevant relations in $H B_{n}(q, Q)$.

(5.1) LEMMA. -

(i) For $i=1, \ldots, n-2, C_{i} C_{i+1} C_{i}-C_{i}=-q^{3} E_{i}$.

(ii) For $i=2, \ldots, n-1, C_{i} C_{i-1} C_{i}-C_{i}=-q^{3} E_{i}$. 
(iii) Let $X=C_{1} C_{0} C_{1}-\kappa C_{1}$, where $\kappa=\frac{q}{Q}+\frac{Q}{q}$. Then $X C_{0}=C_{0} X=q^{-2} Q^{-2} E_{0}$.

(iv) We have $C_{i}^{2}=\delta_{q} C_{i}$ for $i \neq 0$, while $C_{0}^{2}=\delta_{Q} C_{0}$.

The proofs are simple computations in $H B_{n}(q, Q)$.

(5.2) Definition. - The Temperley-Lieb algebra $T L B_{n}(q, Q)$ of type $B_{n}$ with parameters $(q, Q)$ is defined as

$$
T L B_{n}(q, Q):=H B_{n}(q, Q) /\left\langle E_{1}, X\right\rangle=H B_{n}(q, Q) /\left\langle E_{1}, E_{2}, \ldots, E_{n-2}, X\right\rangle
$$

Note that the kernel of the natural map $\widehat{H_{n}^{a}}(q) \rightarrow T L B_{n}(q, Q)$ contains the kernel of the natural map $\widehat{H_{n}^{a}}(q) \rightarrow \widehat{T L_{n}^{a}}(q)$. Hence the former map factors through a surjection

$$
\widehat{T L_{n}^{a}}(q) \rightarrow T L B_{n}(q, Q)
$$

This is reflected in the diagram (5.6) below.

Denote by lower case letters the images in $T L B_{n}(q, Q)$ of the corresponding elements of $\widehat{H_{n}^{a}}(q)$ (or $\widehat{T L_{n}^{a}}(q)$ or $\left.H B_{n}(q, Q)\right)$. Thus in particular we write $t_{i}, c_{i}$ respectively for the images in $T L B_{n}(q, Q)$ of $T_{i}, C_{i} \in H B_{n}(q, Q)$ under the natural map $(i=0,1,2, \ldots, n)$. Similarly, we have elements $x_{i}(i=1, \ldots, n)$ and $v \in T L B_{n}(q, Q)$. The next statement is easy to verify from the foregoing discussion.

(5.3) Proposition. -

(a) The Temperley-Lieb algebra $T L B_{n}(q, Q)$ is generated as R-algebra by the family $\left\{c_{0}, c_{1}, \ldots, c_{n-1}\right\}$ subject to the relations

(TLB)

$$
\begin{aligned}
c_{0}^{2} & =\delta_{Q} c_{0}, \\
c_{i}^{2} & =\delta_{q} c_{i} \quad \text { for } 1 \leqslant i \leqslant n-1, \\
c_{i} c_{i+1} c_{i} & =c_{i} \quad \text { for } 1 \leqslant i \leqslant n-2, \\
c_{i} c_{i-1} c_{i} & =c_{i} \quad \text { for } 2 \leqslant i \leqslant n-1, \\
c_{1} c_{0} c_{1} & =\kappa c_{1},
\end{aligned}
$$

where $\kappa=\frac{q}{Q}+\frac{Q}{q}$ and $\delta_{x}=-\left(x+x^{-1}\right)$ for $x \in R^{\times}$.

(b) The elements $c_{1}, \ldots, c_{n-1}$ generate a subalgebra of $T L B_{n}(q, Q)$ which is isomorphic to the usual Temperley-Lieb algebra $T L_{n}(q)$ of type $\widetilde{A_{n-1}}$.

(c) The following relations hold in $T L B_{n}(q, Q)$.

(i) $t_{0}=-\left(c_{0}+Q^{-1}\right)$ and $t_{i}=-\left(c_{i}+q^{-1}\right)$ for $i=1, \ldots, n-1$.

(ii) If $v \in T L B_{n}(q, Q)$ is the image of $V \in H B_{n}(q, Q)$ under the natural map, then

$$
v=t_{0} t_{1} \ldots t_{n-1}
$$

(iii) $x_{1} x_{2} \ldots x_{n}=v^{n}$. 
A diagram of maps relating the algebras we have now introduced is as follows.

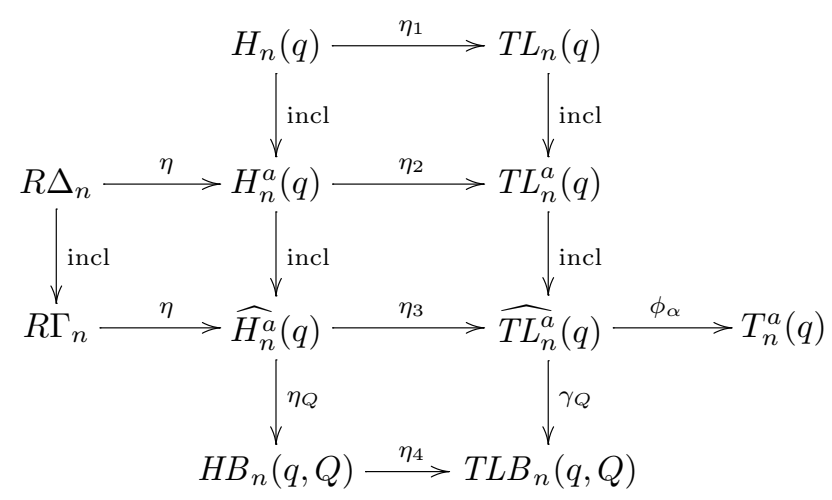

where the $\eta_{i}$ are the natural surjections and $\gamma_{Q}$ exists because the kernel of $\eta_{4} \circ \eta_{Q}$ contains $E_{1} \in \widehat{H_{n}^{a}}(q)$, which generates the kernel of $\eta_{3}$. Thus $\eta_{4} \circ \eta_{Q}$ factors through $\widehat{T L_{n}^{a}}(q)$.

The algebra $T L B_{n}(q, Q)$ has a description in terms of marked diagrams (see $[25,26,29]$ ) which we now describe, because this description is convenient for the discussion of cell modules. We shall use the language of [14] for diagrams. Recall that if $t, n$ are positive integers of the same parity, a finite (planar) diagram $\mu: t \rightarrow n$ is represented by a set of non-intersecting arcs which are contained in the "fundamental rectangle" (see below). These arcs divide the fundamental rectangle into regions, among which there is a unique "left region" as shown below.

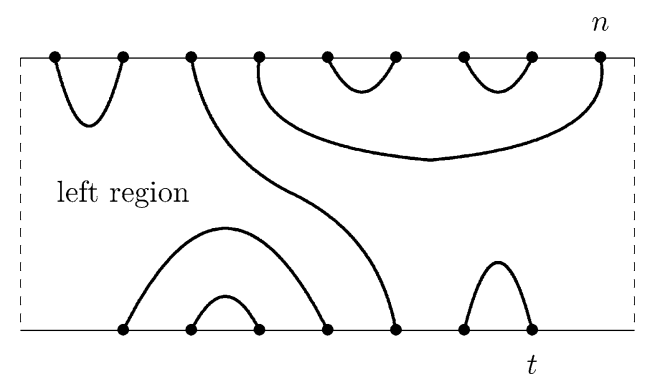

A marked diagram is a (finite planar) diagram, where the interior of the boundary arcs of the leftmost region may be marked with one or more • symbols ("marks") (see below).

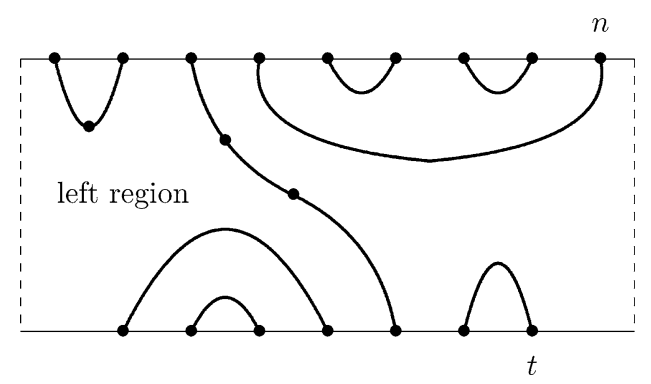

The $R$-linear combinations of diagrams from $t$ to $n$ constitute the morphisms in the Temperley-Lieb category $\mathbb{T}$, where the objects are the non negative integers $\mathbb{Z}_{\geqslant 0}$. Composition 
is defined by concatenation of diagrams, with closed loops being deleted and replaced by the scalar $\delta_{q}$. In particular, $\operatorname{Hom}_{\mathbb{T}}(n, n) \cong T L_{n}(q)$. Marked diagrams may be similarly concatenated according to rules we shall now state; this produces a new category, $\mathbb{T B}$, the Temperley-Lieb category of type $B$. The composition rules are as follows.

A marked diagram is proper if it has no loops and each arc has at most one mark. The following rules reduce the concatenation of any two diagrams to an $R$ linear combination of proper diagrams:

$$
\text { If } \mu \text { is a diagram and } L \text { is a loop with no marks, } \mu \amalg L=\delta_{q} \mu \text {. }
$$

$$
\text { If, in (i), } L \text { has one mark, } \mu \amalg L=\kappa \mu \text {, where } \kappa=\frac{q}{Q}+\frac{Q}{q} \text {. }
$$

If some arc of $\mu$ has more than one mark and $\mu^{\prime}$ is the diagram obtained by removing a mark from the arc concerned, then $\mu=\delta_{Q} \mu^{\prime}$.

Now consider the following marked diagrams from $n$ to $n$.
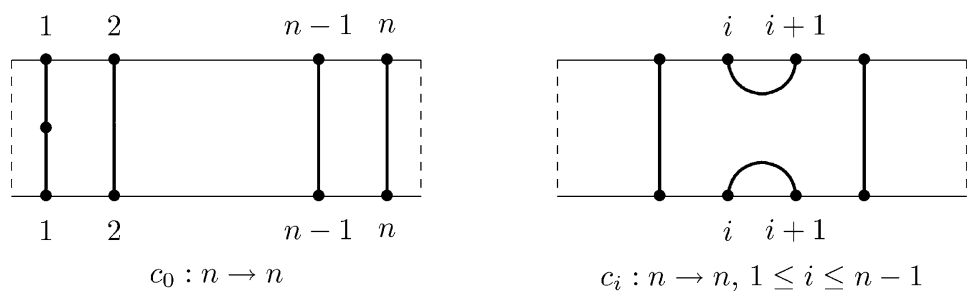

(5.8) Proposition. - The diagrams labelled $c_{0}, c_{1}, \ldots, c_{n-1}$ satisfy the relations for the generators of $T L B_{n}(q, Q)$ given in (5.3). Moreover $T L B_{n}(q, Q)$ is faithfully represented in this way as $\operatorname{End}_{\mathbb{T B}}(n)$ in the category $\mathbb{T B}$.

Proof. - The relations among diagrams $c_{i}$ are easily checked using the rules (5.7). It follows that there is a homomorphism: $T L B_{n}(q, Q) \rightarrow \operatorname{End}_{\mathbb{B}}(n)$, which is easily seen to be surjective, since any proper marked diagram $n \rightarrow n$ can be obtained by composing the $c_{i}$. The injectivity of this homomorphism follows from arguments in [29] or [12].

Let us now focus attention on the bottom right part of the commutative diagram (5.6). Recall that under the map $\eta_{Q}, X_{1} \in \widehat{H_{n}^{a}}(q)$ is mapped to $T_{0} \in T L B_{n}(q, Q)$. But for any element $\mu \in R^{\times}$, we may deform both $\eta_{Q}$ and $\gamma_{Q}$ to $\eta_{Q, \mu}$ and $\gamma_{Q, \mu}$ respectively, where $\eta_{Q, \mu}\left(X_{1}\right)=\mu T_{0}$, $\eta_{Q, \mu}\left(T_{i}\right)=T_{i}$ for $1 \leqslant i \leqslant n-1, \gamma_{Q, \mu}(V)=\mu \gamma_{Q}(V)=\mu t_{0} t_{1} \ldots t_{n-1}$ and $\gamma_{Q, \mu}\left(C_{i}\right)=c_{i}$ for $1 \leqslant i \leqslant n-1$. We then obtain a "deformed" version of the bottom right part of the commutative diagram (5.6) as follows.

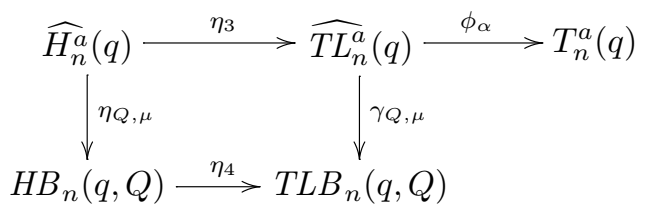

We shall study the pullbacks to $\widehat{H_{n}^{a}}(q)$ of cell modules of $T_{n}^{a}(q)$ by realising the latter as pullbacks of cell modules of $\operatorname{TLB}_{n}(q, Q)$ via a homomorphism yet to be defined. With the 
objective of defining a map: $T_{n}^{a}(q) \rightarrow T L B_{n}(q, Q)$ which makes the diagram (5.9) commute, we shall prove

(5.10) THEOREM. - Let $t_{i}, c_{i}$ and $v$ be the elements of $T L B_{n}(q, Q)$ defined just before (5.3) above. Then for $n \geqslant 2$ we have

$$
c_{1} v^{2}=-q^{-(n-2)} c_{1} c_{2} \ldots c_{n-1} .
$$

Proof. - This will be by induction on $n$. We first verify the statement for $n=2$. The left side of $(5.10 .1)$ is then

$$
\begin{aligned}
c_{1}( & \left.t_{0} t_{1}\right)^{2} \\
= & c_{1} t_{0} t_{1} t_{0} t_{1} \\
= & c_{1} t_{1} t_{0} t_{1} t_{0} \quad \text { since the } t_{i} \text { satisfy the braid relations (BRB) } \\
= & q c_{1} t_{0} t_{1} t_{0} \quad \text { since } c_{i} t_{i}=q c_{i} \\
= & -q c_{1}\left(c_{0}+Q^{-1}\right)\left(c_{1}+q^{-1}\right)\left(c_{0}+Q^{-1}\right) \\
= & -q c_{1}\left(c_{0} c_{1} c_{0}+q^{-1} \delta_{Q} c_{0}+Q^{-1}\left(c_{0} c_{1}+c_{1} c_{0}\right)+2 q^{-1} Q^{-1} c_{0}+Q^{-2} c_{1}+q^{-1} Q^{-2}\right) \\
= & -q\left(\kappa c_{1} c_{0}+q^{-1} \delta_{Q} c_{1} c_{0}+Q^{-1}\left(\kappa c_{1}+\delta_{q} c_{1} c_{0}\right)\right. \\
& \left.+2 q^{-1} Q^{-1} c_{1} c_{0}+Q^{-2} \delta_{q} c_{1}+q^{-1} Q^{-2} c_{1}\right) \\
= & -q c_{1} c_{0}\left(\kappa+q^{-1} \delta_{Q}+Q^{-1} \delta_{q}+2 q^{-1} Q^{-1}\right)-q c_{1}\left(Q^{-1} \kappa+Q^{-2} \delta_{q}+q^{-1} Q^{-2}\right) \\
= & -c_{1},
\end{aligned}
$$

which proves (5.10.1) for the case $n=2$.

Now suppose $n \geqslant 3$. Then

$$
\begin{aligned}
c_{1} v^{2} & =c_{1} t_{0} t_{1} \ldots t_{n-1} t_{0} t_{1} \ldots t_{n-1} \\
& =c_{1} t_{0} t_{1} \ldots t_{n-2} t_{0} t_{1} \ldots t_{n-3} t_{n-1} t_{n-2} t_{n-1} \\
& =c_{1} t_{0} t_{1} \ldots t_{n-2} t_{0} t_{1} \ldots t_{n-3} t_{n-2} t_{n-1} t_{n-2} \quad \text { by the braid relations (BRB) } \\
& =c_{1}\left(t_{0} t_{1} \ldots t_{n-2}\right)^{2} t_{n-1} t_{n-2} \\
& =-q^{-(n-3)} c_{1} c_{2} \ldots c_{n-2} t_{n-1} t_{n-2} \quad \text { by induction on } n \\
& =-q^{-(n-3)} c_{1} c_{2} \ldots c_{n-2}\left(c_{n-1}+q^{-1}\right)\left(c_{n-2}+q^{-1}\right) \\
& =-q^{-(n-3)} c_{1} c_{2} \ldots c_{n-3}\left(c_{n-2}+q^{-1} c_{n-2} c_{n-1}+q^{-1} \delta_{q} c_{n-2}+q^{-2} c_{n-2}\right) \\
& =-q^{-(n-2)} c_{1} c_{2} \ldots c_{n-1} \quad \text { by }(5.3)
\end{aligned}
$$

which completes the proof.

(5.11) COROLlARY. - For each element $\beta \in R$ such that $\beta^{2}=-q^{n-2}$, there is a (unique) surjective homomorphism $g_{\beta}: T_{n}^{a}(q) \rightarrow T L B_{n}(q, Q)$ such that $g_{\beta}\left(f_{i}\right)=c_{i}$ for $i=1,2, \ldots, n-1$, and $g_{\beta}(\tau)=\beta v$. If $\alpha, \mu$ satisfy $\alpha^{-1} \mu=\beta$, then the following diagram commutes.

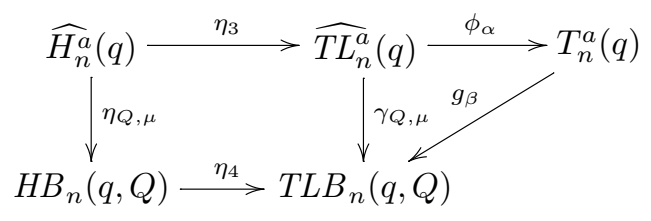


Proof. - For $i=1,2, \ldots, n-1$, the surjection $\gamma_{Q, \mu}$ takes $C_{i} \in \widehat{T L_{n}^{a}}(q)$ to $c_{i} \in T L B_{n}(q, Q)$, while $\gamma_{Q, \mu}(V)=\mu v=\mu t_{0} t_{1} \ldots t_{n-1}$. Hence by Theorem (5.10),

$$
C_{1} V^{2}+\mu^{2} q^{-(n-2)} C_{1} C_{2} \ldots C_{n-1} \in \operatorname{Ker}\left(\gamma_{Q, \mu}\right) .
$$

$\operatorname{But} \operatorname{Ker}\left(\phi_{\alpha}\right)$ is generated by

$$
\nu_{\alpha}=\alpha^{-2} C_{1} V^{2}-C_{1} C_{2} \ldots C_{n-1} .
$$

Hence $\operatorname{Ker}\left(\gamma_{Q, \mu}\right) \supset \operatorname{Ker}\left(\phi_{\alpha}\right)$, provided that

$$
\alpha^{2}=-\mu^{2} q^{-(n-2)}
$$

If this condition is satisfied, $\gamma_{Q, \mu}: \widehat{T L_{n}^{a}}(q) \rightarrow T L B_{n}(q, Q)$ factors through

$$
\phi_{\alpha}: \widehat{T L_{n}^{a}}(q) \rightarrow T_{n}^{a}(q)
$$

i.e., there is a map $g_{\beta}: T_{n}^{a}(q) \rightarrow T L B_{n}(q, Q)$ which makes the diagram (5.11.1) commute. For this map, we have by commutativity, $g_{\beta}\left(f_{i}\right)=\gamma_{Q, \mu}\left(C_{i}\right)=c_{i}$ for $i=1,2, \ldots, n-1$, and $g_{\beta}(\tau)=\gamma_{Q, \mu}\left(\alpha^{-1} V\right)=\beta v$, where $\beta=\alpha^{-1} \mu$ satisfies (5.11.2), i.e. $\beta^{2}=-q^{n-2}$.

Note that the homomorphism $g_{\beta}$ depends of course on $Q$. We shall write $g_{\beta}=g_{\beta, Q}$ when we need to refer to this dependence.

Suppose now that we have a triple $(\alpha, \mu, \beta)$ of elements of $R^{\times}$such that $\beta^{2}=-q^{n-2}$ and $\beta=\alpha^{-1} \mu$. By (5.11), we have a commutative diagram

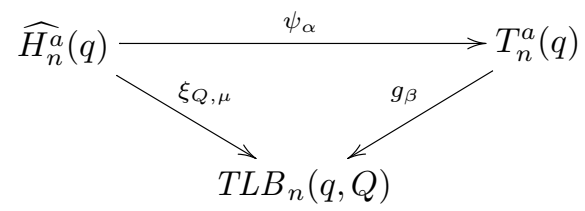

where, in the notation of (5.11.1), $\psi_{\alpha}=\phi_{\alpha} \circ \eta_{3}$ and $\xi_{Q, \mu}=\gamma_{Q, \mu} \circ \eta_{3}=\eta_{4} \circ \eta_{Q, \mu}$.

For future reference, we summarise the definitions of the maps in (5.12) in the following equations.

$$
\begin{aligned}
\xi_{Q, \mu}\left(X_{1}\right) & =\mu t_{0}, \\
\xi_{Q, \mu}\left(T_{i}\right) & =t_{i} \quad \text { for } i=1,2, \ldots, n-1, \\
\psi_{\alpha}\left(V=X_{1} T_{1} \ldots T_{n-1}\right) & =\alpha \tau \\
\psi_{\alpha}\left(C_{i}=-\left(T_{i}+q^{-1}\right)\right) & =f_{i} \quad \text { for } i=1,2, \ldots, n-1, \\
g_{\beta}\left(f_{i}\right) & =c_{i}=-\left(t_{i}+q^{-1}\right) \text { for } i=1,2, \ldots, n-1, \\
g_{\beta}(\tau) & =\beta v=\beta t_{0} t_{1} \ldots t_{n-1} .
\end{aligned}
$$

Our next objective is to identify the pullbacks via $\psi_{\alpha}$ of the cell modules $W_{t, z}(n)$ of $T_{n}^{a}(q)$ as standard modules $M_{s, N}$ of $\widehat{H_{n}^{a}}(q)$ up to Grothendieck equivalence. We shall do this 
by identifying $W_{t, z}(n)$ as the pullback of a cell module $W_{t}(n)$ of $T L B_{n}(q, Q)$ via $g_{\beta}$ for appropriate $Q$, and using the commutative diagram (5.12). We therefore need to discuss the cellular structure of $T L B_{n}(q, Q)$, which we do in the next section.

\section{Cell modules for $T_{n}^{a}(q)$ and $T L B_{n}(q, Q)$}

The cell modules $W_{t, z}(n)$ of $T_{n}^{a}(q)$ were defined and analysed in [14]. We shall freely use the notation and language of that work here. There is an entirely analogous "cellular theory" for the algebras $T L B_{n}(q, Q)$ which we shall now sketch. Recall [13] that to specify a cellular structure for $T L B_{n}(q, Q)$, we require (i) a poset $\mathcal{T}$, (ii) for each $t \in \mathcal{T}$, a set $M(t)$, and (iii) an injection $\amalg_{t \in \mathcal{T}} M(t) \times M(t) \stackrel{C}{\rightarrow} T L B_{n}(q, Q)$, whose image is an $R$-basis $\left\{C_{S, T}^{t}\right\}$ of $T L B_{n}(q, Q)$ which satisfies

$$
\begin{aligned}
& \left.a C_{S, T}^{t}=\sum_{S^{\prime} \in M(t)} r_{a}\left(S^{\prime}, S\right) C_{S^{\prime}, T}^{t}+\text { lower terms (corresponding to } t^{\prime}<t\right) \\
& \left(a \in T L B_{n}(q, Q), r_{a}\left(S^{\prime}, S\right) \in R\right) .
\end{aligned}
$$

We now specify the various elements of a cellular structure for $T L B_{n}(q, Q)$. Take

$$
\mathcal{T}=\{t \in \mathbb{Z}|| t \mid \leqslant n, t \equiv n(\bmod 2)\},
$$

partially ordered as follows: $t \leqslant s$ if $|t|<|s|$ or $|t|=|s|$ and $t \leqslant s$.

To define $M(t)$, first take $t \in \mathcal{T}, t \geqslant 0$. Then $M(t)$ is the set of monic diagrams $D: t \rightarrow n$ with no marked through strings, where "monic" means that there are $t$ through strings, as in [14], where it is shown that this is equivalent to $D$ being a monic morphism in the category-theoretic sense. In general, let $M(t)=M(|t|)$. Then $C: M(t) \times M(t) \rightarrow T L B_{n}(q, Q)$ is defined as follows. Let $S, T \in M(t)$. For $t \geqslant 0$, define $C_{S, T}^{t}=S \circ T^{*}$, where ${ }^{*}$ denotes reflection in a horizontal axis. For $t<0$, define $C_{S, T}^{t}=S \circ c_{o} \circ T^{*}$, where $c_{0}=c_{0}(t): t \rightarrow t$ is the generator shown above (5.8). This is the diagram $S \circ T^{*}$, with the leftmost through string marked. The cellular axioms above are easily checked.

The cell modules $W_{t}(n)$ are now defined in complete analogy with the $W_{t, z}(n)$ of [14]. For any $t \in \mathcal{T}, W_{t}(n)$ has basis $M(t)$. If $t \geqslant 0, T L B_{n}(q, Q)$ acts via composition in the category $\mathbb{T B}$; explicitly, if $D \in M(t)$ and $\omega \in T L B_{n}(q, Q)$, then $\omega \cdot D=\omega D$ (composition in $\mathbb{T B}$ ) if $\omega \circ D \in M(t)$, and $\omega \cdot D=0$ otherwise.

For $t<0$, one may think of $W_{t}(n)$ as having basis the set $\left\{D \circ c_{0}(t) \mid D \in M(|t|)\right\}$ of monic diagrams $t \rightarrow n$ in $\mathbb{T B}$ with the leftmost through string marked. Then the action of $T L B_{n}(q, Q)$ is essentially multiplication in $\mathbb{T B}$, as in the case $t \geqslant 0$. Thus if $t<0$, then $\omega$. $\left(D \circ c_{0}(t)\right)=0$ if $\omega \circ D$ is not monic, while $\omega .\left(D \circ c_{0}\right)=\omega D c_{0}$ (composition in $\mathbb{T} \mathbb{B}$ ) if $\omega \circ D$ is monic.

It is easily seen (cf. [29]) that the dimension (i.e. rank over $R$ ) of $W_{t}(n)$ is $\left(\begin{array}{c}n \\ \frac{n-|t|}{2}\end{array}\right)$, which is the same as that of $W_{t, z}(n)$. We now wish to identify the inflation of $W_{t}(n)$ via the surjection $g_{\beta}: T_{n}^{a}(q) \rightarrow T L B_{n}(q, Q)$.

(6.1) THEOREM. - Suppose $R$ is any commutative ring and suppose that $q, Q$ are elements of $R^{\times}$, and that $\delta_{q}=-\left(q+q^{-1}\right)$ is not a zero-divisor. Let $\beta \in R^{\times}$satisfy $\beta^{2}=-q^{n-2}$ and let $g_{\beta, Q}=g_{\beta}: T_{n}^{a}(q) \rightarrow T L B_{n}(q, Q)$ be the surjection defined in (5.11). For $t \in \mathbb{Z}$ such that $0 \leqslant t \leqslant n$ and $t \equiv n(\bmod 2)$, write $z_{t}=(-1)^{t} \beta Q^{-1} q^{-\frac{1}{2}(n+t-2)}$. If $W_{t}(n)$ is the cell module for $T L B_{n}(q, Q)$ described above, then the inflation $g_{\beta}^{*} W_{t}(n)$ (i.e. the pullback via $g_{\beta}$ of $W_{t}(n)$ ) is isomorphic to $W_{t, z}(n)$, where $z=z_{t}$.

The proof will depend on a sequence of lemmas. 
(6.2) Remark. - Observe first that the finite rank Temperley-Lieb algebra $T L_{n}(q)$ is naturally a subalgebra of both $T_{n}^{a}(q)$ and $T L B_{n}(q, Q)$; in $T_{n}^{a}(q)$ it is generated by $f_{1}, \ldots, f_{n-1}$, and has $R$-basis consisting of "finite diagrams" from $n$ to $n$, i.e., diagrams with no arcs crossing the boundary of the fundamental rectangle (see $[14, \S 2])$. In $T L B_{n}(q, Q)$, it is generated by $c_{1}, \ldots, c_{n-1}$ and has basis consisting of unmarked diagrams from $n$ to $n$. These two sets of diagrams may be identified in the obvious way, and relations (5.13) show that $g_{\beta}$ maps a diagram in $T L_{n}(q) \subset T_{n}^{a}(q)$ to the same diagram, regarded as a diagram in $T L B_{n}(q, Q)$. A similar observation applies more generally to affine and marked diagrams $t$ to $n$, where finite and unmarked diagrams respectively may be identified with each other.

Recall [14] that an affine diagram from $t$ to $n$ is monic if it has $t$ through strings. Let $X_{t}$ be the $T_{n}^{a}(q)$-module with basis all monic affine diagrams $: t \rightarrow n$, with $T_{n}^{a}(q)$ action given by composition in the category $\mathbb{T}^{a}$, modulo diagrams with fewer than $t$ through strings. Thus $X_{t}$ may be thought of as a quotient of the left $T_{n}^{a}(q)$-module $\operatorname{Hom}_{\mathbb{T}^{a}}(t, n)$ by the submodule spanned by diagrams with fewer than $t$ through strings. The $T_{n}^{a}(q)$ module $W_{t, z}(n)$ is defined as the quotient of $X_{t}$ by the ideal

$$
I_{\chi}:=\left\{\gamma \tau_{t}-\chi \gamma\right\}
$$

for $\gamma \in X_{t}$, where $\chi=\chi_{z}=z$ if $t \neq 0$ and $\chi=\chi_{z}=z+z^{-1}$ if $t=0$.

We shall define a homomorphism $\theta: X_{t} \rightarrow g_{\beta}^{*} W_{t}(n)$ which we shall prove factors through $W_{t, z}(n)$ for appropriate $z$. To do this, we require the following diagrams, which, being finite, may (and will) be alternately thought of as lying in the categories $\mathbb{T}^{a}$ and $\mathbb{T B}$.

(6.4) Definition. - First suppose $t>0$. Define monic finite diagrams $D_{t}, D_{t}^{\prime}: t \rightarrow n$ as depicted below.
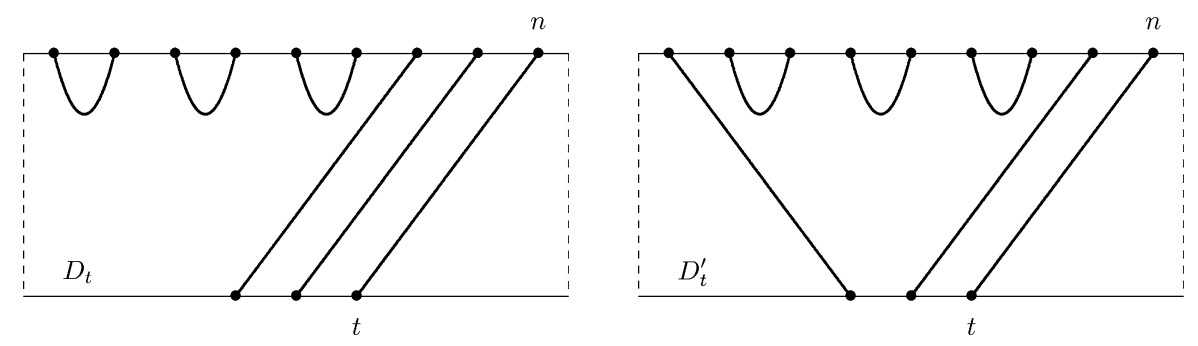

In $D_{t},(t>0)$ the $t$ through strings are joined to the rightmost $t$ top nodes, while in $D_{t}^{\prime}$ they are joined to the rightmost $t-1$ and leftmost top nodes. Note that $D_{t}$ and $D_{t}^{\prime}$ are finite diagrams and that

$$
D_{t}^{*} D_{t}^{\prime}=\left(D_{t}^{\prime}\right)^{*} D_{t}=\mathrm{id}_{t} .
$$

When $t=0$ the corresponding diagrams are as below.
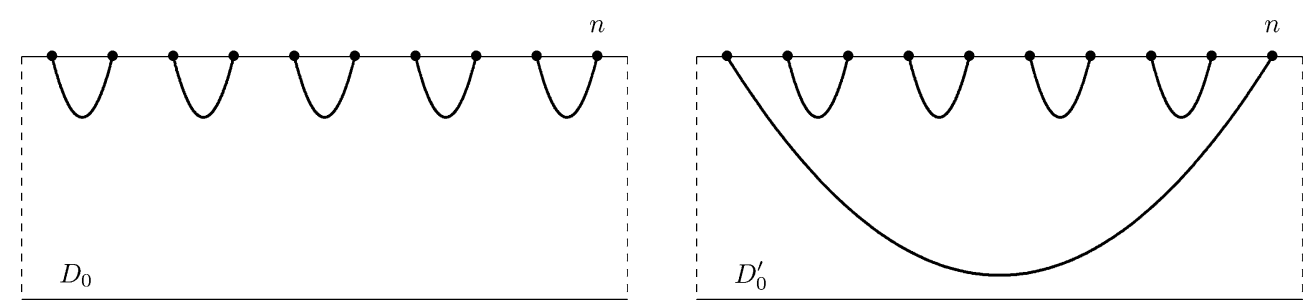
In this case

$$
\left(D_{0}^{\prime}\right)^{*} D_{0}=D_{0}^{*} D_{0}^{\prime}=\delta_{q} \operatorname{id}_{0},
$$

(6.5) Definition. - Let $X_{t}$ be the $T_{n}^{a}(q)$-module with basis the set of all monic diagrams $t \rightarrow n$ (as explained above). Define $\theta: X_{t} \rightarrow Z_{t}:=g_{\beta}^{*} W_{t}(n)$ by the formula

$$
\theta(\gamma)=g_{\beta}\left(\gamma D_{t}^{*}\right) D_{t}^{\prime}
$$

where $\gamma \in X_{t}$, and $D_{t}^{*}$ and $D_{t}^{\prime}$ are regarded as diagrams in $\mathbb{T}^{a}$ and $\mathbb{T B}$ respectively (see Remark (6.2)).

(6.6) LEMMA. -

(i) (cf. Remark (6.2)) If $\gamma \in X_{t}$ is a finite diagram, then provided that $t>0$, we have $\theta(\gamma)=\gamma$; i.e., $\theta(\gamma)$ is the same diagram as $\gamma$, regarded as a marked diagram in $W_{t}(n)$. If $t=0$, then

$$
\theta(\gamma)=\delta_{q} \gamma
$$

(ii) Suppose that for some element $\chi \in R$, we know that $\theta\left(\gamma \tau_{t}\right)=\chi \theta(\gamma)$ for all finite diagrams $\gamma \in X_{t}$. Then

$$
\theta\left(\gamma \tau_{t}\right)=\chi \theta(\gamma)
$$

for all $\gamma \in X_{t}$; i.e., $\operatorname{Ker}(\theta) \supset I_{\chi}$.

Proof. - Suppose $\gamma \in X_{t}$ is a finite diagram. Then $\gamma D_{t}^{*}$ is also finite, and hence is in $T L_{n}(q) \subset T_{n}^{a}(q)$. By Remark (6.2), any diagram in $T L_{n}(q)$ is mapped by $g_{\beta}$ to the same diagram, regarded as an element of $T L B_{n}(q, Q)$, whence $\theta(\gamma)=\gamma D_{t}^{*} D_{t}^{\prime}=\gamma$, which proves (i) for $t>0$. The same argument may be used for the case $t=0$, taking into account that $D_{0}^{*} D_{0}^{\prime}=\delta_{q} \mathrm{id}_{0}$.

(ii) Clearly any diagram $\gamma \in X_{t}$ has a factorisation $\gamma=\omega \gamma_{0}$, where $\gamma_{0} \in X_{t}$ is finite, and $\omega$ is a diagram in $T_{n}^{a}(q)$ (in fact more is true: $X_{t}$ is cyclic as $T_{n}^{a}(q)$-module, generated by any one of many finite diagrams). Hence

$$
\begin{aligned}
\theta\left(\gamma \tau_{t}\right) & =\theta\left(\omega \gamma_{0} \tau_{t}\right) \\
& =g_{\beta}(\omega) \theta\left(\gamma_{0} \tau_{t}\right) \quad \text { since } \theta \text { is a } T_{n}^{a}(q) \text {-module homomorphism } \\
& =\chi g_{\beta}(\omega) \theta\left(\gamma_{0}\right) \\
& =\chi \theta\left(\omega \gamma_{0}\right) \\
& =\chi \theta(\gamma),
\end{aligned}
$$

as required.

(6.7) COROLlaRY. - The homomorphism

$$
\theta: X_{t} \rightarrow g_{\beta}^{*} W_{t}(n)=Z_{t}
$$

of $T_{n}^{a}(q)$ modules is surjective if $t>0$, and has image precisely $\delta_{q} Z_{t}$ when $t=0$.

Proof. - By (6.6), if $t>0$, the image of $\theta$ contains all finite diagrams $t \rightarrow n$. But these generate $W_{t, z}(n)$ as $T_{n}^{a}(q)$-module, whence the first statement. If $t=0$, then again by (6.6), for any finite diagram $\gamma, \theta(\gamma) \in \delta_{q} Z_{t}$. Since the finite diagrams generate $Z_{t}$ as $T_{n}^{a}(q)$-module, it follows that the image is contained in $\delta_{q} Z_{t}$. But since the image contains $\delta_{q} \gamma$ for each finite diagram, the result is clear. 
We shall show that $\theta$ factors through $W_{t, z}(n)$ for appropriate $z \in R^{\times}$using the method of (6.6)(ii), i.e. by showing that $\operatorname{Ker}(\theta) \supset I_{\chi}$ for $\chi=\chi_{z}, z=z_{t}$. We therefore investigate the right action of $\tau_{t}$ on $X_{t}$.

Recall (5.13) that $g_{\beta}\left(\tau_{n}\right)=\beta t_{0} t_{1} \ldots t_{n-1}$, where $\tau_{n}$ is thought of as an element of $T_{n}^{a}(q)$. Thus to carry through the strategy of (6.6), we shall need the following computation in $\mathbb{T B}$. We adopt the following notation for diagrams in $\mathbb{T B}$ : we say that a diagram is positive if the leftmost through string is unmarked, and negative otherwise. If $E$ is a positive diagram (e.g. in $W_{t}(n)$ ), $\dot{E}$ will denote the diagram obtained from $E$ by marking the leftmost through string.

(6.8) Lemma. - Suppose $t>0$. Then in the category $\mathbb{T} \mathbb{B}$, we have

$$
\beta t_{n-1} t_{n-2} \ldots t_{1} t_{0} D_{t}^{\prime}=z_{t}\left(D_{t}+Q \dot{D}_{t}+E\right)
$$

where $D_{t}, D_{t}^{\prime}$ are the diagrams defined above and $E$ is a linear combination of diagrams with $<t$ through strings. The right side of this equation is to be thought of as an element of $\operatorname{Hom}_{\mathbb{T} B}(t, n)$.

Proof. - Recall that for $1 \leqslant i \leqslant n-1, t_{i}=-\left(c_{i}+q^{-1}\right)$, while $t_{0}=-\left(c_{0}+Q^{-1}\right)$. Hence $t_{0} D_{t}^{\prime}=-\dot{D}_{t}^{\prime}-Q^{-1} D_{t}^{\prime}$. Thus we need to evaluate $t_{n-1} t_{n-2} \ldots t_{1} D_{t}^{\prime}$ and $t_{n-1} t_{n-2} \ldots t_{1} \dot{D}_{t}^{\prime}$. For the former, we have $t_{1} D_{t}^{\prime}=-D_{t}^{\prime}(n-2)-q^{-1} D_{t}^{\prime}$, where $D_{t}^{\prime}(n-2)$ is the diagram obtained from $D_{t}^{\prime}$ by moving the leftmost through string past the leftmost horizontal arc. Now $c_{2} D_{t}^{\prime}(n-2)=D_{t}^{\prime}$ and $c_{2} D_{t}^{\prime}=\delta_{q} D_{t}^{\prime}$, from which it follows that

$$
t_{2} t_{1} D_{t}^{\prime}=q^{-1} D_{t}^{\prime}(n-2) .
$$

Repeating this argument $k$ times, where $2 k=n-t$, we obtain

$$
t_{2 k} t_{2 k-1} \ldots t_{2} t_{1} D_{t}^{\prime}=q^{-k} D_{t} .
$$

But for $i>2 k$ one sees easily that $t_{i} D_{t} \equiv-q^{-1} D_{t}$ modulo diagrams with fewer than $t$ through strings. Hence

$$
\begin{aligned}
t_{n-1} t_{n-2} \ldots t_{1} D_{t}^{\prime} & =(-1)^{t-1} q^{-t-k+1} D_{t}+E_{1} \\
& =(-1)^{t-1} q^{-\frac{n+t-2}{2}} D_{t}+E_{1}
\end{aligned}
$$

where $E_{1}$ is a linear combination of diagrams with $<t$ through strings. The same computation shows that

$$
\begin{aligned}
t_{n-1} t_{n-2} \ldots t_{1}\left(\dot{D}_{t}^{\prime}\right) & =(-1)^{t-1} q^{-t-k+1}\left(\dot{D}_{t}\right)+E_{2} \\
& =(-1)^{t-1} q^{-\frac{n+t-2}{2}}\left(\dot{D}_{t}\right)+E_{2}
\end{aligned}
$$

where $E_{2}$ is a linear combination of diagrams with $<t$ through strings. It follows that

$$
\begin{aligned}
\beta t_{n-1} t_{n-2} \ldots t_{1} t_{0} D_{t}^{\prime} & =\beta t_{n-1} t_{n-2} \ldots t_{1}\left(t_{0} D_{t}^{\prime}=-\left(\dot{D}_{t}^{\prime}\right)-Q^{-1} D_{t}^{\prime}\right) \\
& =\beta(-1)^{t}\left(q^{-\frac{n+t-2}{2}}\left(\dot{D}_{t}\right)+Q^{-1} q^{-\frac{n+t-2}{2}} D_{t}\right)+E \\
& =z_{t} D_{t}+Q z_{t}\left(\dot{D}_{t}\right)+E,
\end{aligned}
$$

where $E$ is a combination of diagrams with $<t$ through strings.

We now turn to the case $t=0$.

Recall that the diagrams $D_{0}, D_{0}^{\prime}$ have been defined above, before the statement of Lemma (6.4). The following relations are easily verified. 


$$
\begin{aligned}
\left(D_{0}^{\prime}\right)^{*} D_{0} & =\left(D_{0}\right)^{*} D_{0}^{\prime}=\delta_{q} \operatorname{id}_{0}, \quad \text { and } \\
\left(c_{0} D_{0}^{\prime}\right)^{*} D_{0} & =\left(D_{0}^{\prime}\right)^{*} c_{0} D_{0}=\kappa \operatorname{id}_{0}=\left(\frac{Q}{q}+\frac{q}{Q}\right) \operatorname{id}_{0}
\end{aligned}
$$

where $\mathrm{id}_{0}$ is the empty diagram $0 \rightarrow 0$, in either the category $\mathbb{T}^{a}$ or $\mathbb{T B}$. Note that although in the discussion above it is always assumed that $n \geqslant 1$, from the point of view of diagram algebras, the point 0 is an object in both categories $\mathbb{T}^{a}$ and $\mathbb{T B}$. The corresponding algebras are $T L B_{0}(q, Q)=R . \mathrm{id}_{0} \cong R$ and $T_{0}^{a}(q)=R\left\langle\tau_{0}\right\rangle \cong R \mathbb{N}$.

(6.10) Lemma. - Let $\gamma \in X_{0}$ be a finite diagram. Then

$$
\theta\left(\gamma \tau_{0}\right)=\beta \delta_{q} \gamma\left(D_{0}^{\prime}\right)^{*} t_{0} t_{1} \ldots t_{n-1} D_{0}^{\prime} .
$$

Proof. - First, observe that in the category $\mathbb{T}^{a}$, we have the following relation:

$$
\tau_{0}=D_{0}^{*} \tau_{n} D_{0}=\left(D_{0}^{\prime}\right)^{*} \tau_{n} D_{0}^{\prime} .
$$

It follows that for finite $\gamma \in X_{0}$,

$$
\begin{aligned}
\theta\left(\gamma \tau_{0}\right) & =g_{\beta}\left(\gamma \tau_{0} D_{0}^{*}\right) D_{0}^{\prime} \\
& =g_{\beta}\left(\gamma\left(D_{0}^{\prime}\right)^{*} \tau_{n} D_{0}^{\prime} D_{0}^{*}\right) D_{0}^{\prime} \quad \text { by }(6.10 .1) \\
& =g_{\beta}\left(\gamma\left(D_{0}^{\prime}\right)^{*} \tau_{n}\right) g_{\beta}\left(D_{0}^{\prime} D_{0}^{*}\right) D_{0}^{\prime} \\
& =g_{\beta}\left(\gamma\left(D_{0}^{\prime}\right)^{*} \tau_{n}\right) D_{0}^{\prime} D_{0}^{*} D_{0}^{\prime} \quad \text { since } D_{0}^{\prime} D_{0}^{*} \text { is finite } \\
& =\delta_{q} g_{\beta}\left(\gamma\left(D_{0}^{\prime}\right)^{*} \tau_{n}\right) D_{0}^{\prime} \quad \text { by }(6.9) \\
& =\delta_{q} g_{\beta}\left(\gamma\left(D_{0}^{\prime}\right)^{*}\right) g_{\beta}\left(\tau_{n}\right) D_{0}^{\prime} \\
& =\delta_{q} \beta \gamma\left(D_{0}^{\prime}\right)^{*} t_{0} t_{1} \ldots t_{n-1} D_{0}^{\prime} \quad \text { since } \gamma\left(D_{0}^{\prime}\right)^{*} \text { is finite }
\end{aligned}
$$

(6.11) LEMMA. - We have

$$
t_{1} t_{2} \ldots t_{n-1} D_{0}^{\prime}=q^{-\frac{n-4}{2}} D_{0}
$$

Proof. - Denote by $D_{0}^{\prime}(n-2)$ the diagram from 0 to $n$ which looks like $D_{0}^{\prime}: 0 \rightarrow n-2$, with the rightmost top vertices joined by a horizontal arc. Then clearly $c_{n-1} D_{0}^{\prime}=D_{0}^{\prime}(n-2)$. Further, $c_{n-2} D_{0}^{\prime}=\delta_{q} D_{0}^{\prime}$, and $c_{n-2} D_{0}^{\prime}(n-2)=D_{0}^{\prime}$. Thus

$$
t_{n-1} D_{0}^{\prime}=-\left(c_{n-1}+q^{-1}\right) D_{0}^{\prime}=-\left(D_{0}^{\prime}(n-2)+q^{-1} D_{0}^{\prime}\right) .
$$

Hence

$$
\begin{aligned}
t_{n-2} t_{n-1} D_{0}^{\prime} & =\left(c_{n-2}+q^{-1}\right)\left(D_{0}^{\prime}(n-2)+q^{-1} D_{0}^{\prime}\right) \\
& =D_{0}^{\prime}+q^{-1} \delta_{q} D_{0}^{\prime}+q^{-1} D_{0}^{\prime}(n-2)+q^{-2} D_{0}^{\prime} \\
& =q^{-1} D_{0}^{\prime}(n-2) .
\end{aligned}
$$

Repeating this argument $\frac{n-2}{2}$ times, we obtain

$$
t_{1} t_{2} \ldots t_{n-1} D_{0}^{\prime}=q^{-\frac{n-2}{2}} t_{1} D_{0} .
$$

But $c_{1} D_{0}=\delta_{q} D_{0}$, whence $t_{1} D_{0}=-\left(\delta_{q}+q^{-1}\right) D_{0}=q D_{0}$, and the lemma follows. 
Proof of Theorem (6.1). - We show first that for any element $\gamma \in X_{t}(t \geqslant 0)$, we have

$$
\theta\left(\gamma \tau_{t}\right)=\chi_{z} \theta(\gamma)
$$

where $z=z_{t}=(-1)^{t} \beta Q^{-1} q^{-\frac{1}{2}(n+t-2)}$ and

$$
\chi_{z}= \begin{cases}z & \text { if } t>0 \\ z+z^{-1} & \text { if } t=0\end{cases}
$$

By Lemma (6.6)(ii), it suffices to prove (6.1.1) for any finite diagram $\gamma \in X_{t}$.

We prove (6.1.1) first for $t>0$. Recall that if $E$ is a positive morphism (diagram) in $\mathbb{T B}$, $\dot{E}$ is obtained from $E$ by marking the leftmost through string. Then observe that in the above notation, $D_{t}^{*} D_{t}^{\prime}=\mathrm{id}_{t}$, while $\dot{D}_{t}^{*} D_{t}^{\prime}=\dot{\mathrm{id}}_{t}$, and if we regard $W_{t}(n)$ as a quotient of the bimodule $\operatorname{Hom}_{\mathbb{T B}}(t, n)$ in the obvious way, then in $W_{t}(n), \gamma \mathrm{id}_{t}=\gamma$, while $\gamma$ id $_{t}=0$. Moreover, if $E \in \mathbb{T} \mathbb{B}$ is a linear combination of diagrams with fewer that $t$ through strings, then $\gamma E=0$ by definition. Hence

$$
\begin{aligned}
\theta\left(\gamma \tau_{t}\right) & =g_{\beta}\left(\gamma \tau_{t} D_{t}^{*}\right) D_{t}^{\prime} \quad \text { by }(6.5 .1) \\
& =g_{\beta}\left(\gamma\left(D_{t}^{\prime}\right)^{*} \tau_{n}\right) D_{t}^{\prime} \quad \text { since } \tau_{t} D_{t}^{*} \tau_{n}^{-1}=\left(D_{t}^{\prime}\right)^{*} \\
& =g_{\beta}\left(\gamma\left(D_{t}^{\prime}\right)^{*}\right) g_{\beta}\left(\tau_{n}\right) D_{t}^{\prime} \\
& =\beta \gamma\left(D_{t}^{\prime}\right)^{*} t_{0} t_{1} \ldots t_{n-1} D_{t}^{\prime} \quad \text { by }(6.5 .1) \text { again, and (5.13) } \\
& =\gamma\left(\beta t_{n-1} t_{n-2} \ldots t_{1} t_{0} D_{t}^{\prime}\right)^{*} D_{t}^{\prime} \quad \text { since }{ }^{*} \text { is an anti-involution } \\
& =\gamma \chi_{z}\left(D_{t}+Q\left(\dot{D}_{t}\right)+E\right)^{*} D_{t}^{\prime} \quad \text { by }(6.8) \\
& =\chi_{z} \theta(\gamma)
\end{aligned}
$$

which proves (6.1.1) for $t>0$. Now take $t=0$ and repeat the above computation using Lemmas (6.10), (6.11) instead of (6.8). One obtains

$$
\begin{aligned}
\theta\left(\gamma \tau_{0}\right) & =\beta \delta_{q} \gamma\left(D_{0}^{\prime}\right)^{*} t_{0} t_{1} \ldots t_{n-1} D_{0}^{\prime} \quad \text { by }(6.10) \\
& =\beta \delta_{q} \gamma\left(D_{0}^{\prime}\right)^{*} t_{0} q^{-\frac{n-4}{2}} D_{0} \quad \text { by }(6.11) \\
& =-\beta \delta_{q} q^{-\frac{n-4}{2}} \gamma\left(D_{0}^{\prime}\right)^{*}\left(c_{0}+Q^{-1}\right) D_{0} \\
& =-\beta \delta_{q} q^{-\frac{n-4}{2}} \gamma\left(\kappa \mathrm{id}_{0}+Q^{-1} \delta_{q} \mathrm{id}_{0}\right) \\
& =-\left(\frac{Q}{q}-Q^{-1} q^{-1}\right) \beta \delta_{q} q^{-\frac{n-4}{2}} \gamma \\
& =-\left(Q-Q^{-1}\right) \beta q^{-\frac{n-2}{2}} \theta(\gamma) \quad \text { by Corollary (6.7)(ii). }
\end{aligned}
$$

But $z_{0}=\beta Q^{-1} q^{-\frac{n-2}{2}}$, and since $\beta^{2}=-q^{n-2}, \beta^{-1}=-q^{-(n-2)} \beta$. It follows easily that $\theta\left(\gamma \tau_{0}\right)=\left(z_{0}+z_{0}^{-1}\right) \theta(\gamma)$, which completes the proof of (6.1.1).

It follows from (6.1.1) (cf. (6.7)) that $\theta$ induces a homomorphism which we also denote by $\theta: W_{t, z}(n) \rightarrow Z_{t}$, where $z=z_{t}$. Note that $W_{t, z}(n)$ and $Z_{t}$ are free $R$-modules of the same rank. To complete the proof of the theorem, we discuss the cases $t>0$ and $t=0$ separately.

If $t>0$, then by (6.7), $\theta$ is surjective. It follows, since $W_{t, z}(n)$ and $Z_{t}$ are free $R$-modules of the same rank, that $\theta$ is an isomorphism. If $t=0$, the same argument (using (6.7)) shows that $\theta$ defines an isomorphism $W_{t, z}(n) \rightarrow \delta_{q} Z_{t}$. But since $\delta_{q}$ is not a zero-divisor, $\delta Z_{t} \cong Z_{t}$ as $T_{n}^{a}(q)$-modules, which completes the proof. 
Theorem (6.1) shows in particular that any cell module $W_{t, z}(n)$ for the $R$-algebra $T_{n}^{a}(q)$ may be realised as the pullback of a cell module of the algebra $T L B_{n}(q, Q)$.

(6.12) COROLLARY. - For any (relevant) pair $t, z$, the cell module $W_{t, z}(n)$ may be realised as $g_{\beta, Q}^{*} W_{t}(n)$, where $Q=Q(t, z)=(-1)^{t} \beta z^{-1} q^{-\frac{1}{2}(n+t-2)}$.

Recall from the beginning of this section that we also have cell modules $W_{t}(n)$ for $T L B_{n}(q, Q)$, for $t<0$. We next identify their lift to $T_{n}^{a}(q)$ via $g_{\beta, Q}$.

(6.13) TheOREM. - Suppose $\delta_{q}$ is not a zero divisor in $R$ and let $t$ satisfy $0 \leqslant t \leqslant n$, $t \equiv n(\bmod 2)$. Then the inflation

$$
g_{\beta}{ }^{*} W_{-t}(n) \cong W_{t, q^{-t} z_{t}^{-1}}(n)=W_{t,-Q^{2} z_{t}}(n)
$$

where $z_{t}=(-1)^{t} \beta Q^{-1} q^{-\frac{1}{2}(n+t-2)}$ as in (6.1).

Proof. - The case $t=0$ has been treated above. Note that $z_{0}=\beta Q^{-1} q^{-\frac{1}{2}(n-2)}$, so that $z_{0}^{2}=-Q^{-2}$. Thus $-Q^{2} z_{0}=z_{0}^{-1}$, and since $W_{0, z}(n)=W_{0, z^{-1}}(n)$, the case $t=0$ follows from Theorem (6.1). We therefore take $t>0$.

First observe that in analogy with (6.5), we have a homomorphism of $T_{n}^{a}(q)$-modules $\theta^{-}: X_{t} \rightarrow g_{\beta}{ }^{*} W_{-t}(n)$ given by

$$
\theta^{-}(\gamma)=g_{\beta}\left(\gamma D_{t}^{*}\right)\left(\dot{D}_{t}^{\prime}\right)
$$

where $\gamma \in X_{t}$, and $D_{t}^{*}$ and $\dot{D}_{t}^{\prime}=D_{t}^{\prime} \circ c_{0}(t)$ are the diagrams defined in the proof of (6.3), regarded as diagrams in $\mathbb{T}^{a}$ and $\mathbb{T B}$ respectively (see immediately preceding (6.8)) that for a diagram $E$ with unmarked through strings, $\dot{E}$ denotes the same diagram with the leftmost through string marked. One verifies easily that for a finite diagram $\gamma: t \rightarrow n\left(\in X_{t}\right)$ we have

$$
\theta^{-}(\gamma)=(\dot{\gamma})
$$

In accordance with the strategy of the proof of Theorem (6.1), to prove the theorem it will suffice to show that for any finite diagram $\gamma \in X_{t}$, we have

$$
\theta^{-}\left(\gamma \tau_{t}\right)=q^{-t} z_{t}^{-1} \theta^{-}(\gamma)=q^{-t} z_{t}^{-1}(\dot{\gamma})
$$

Arguing as in the proof of (6.1), we have

$$
\begin{aligned}
\theta^{-}\left(\gamma \tau_{t}\right) & =g_{\beta}\left(\gamma \tau_{t} D_{t}^{*}\right)\left(\dot{D}_{t}^{\prime}\right) \\
& =g_{\beta}\left(\gamma\left(D_{t}^{\prime}\right)^{*} \tau_{n}\right)\left(\dot{D}_{t}^{\prime}\right) \quad \text { since } \tau_{t} D_{t}^{*} \tau_{n}^{-1}=\left(D_{t}^{\prime}\right)^{*} \\
& =\gamma\left(D_{t}^{\prime}\right)^{*} g_{\beta}\left(\tau_{n}\right)\left(\dot{D}_{t}^{\prime}\right) \\
& =\gamma\left(D_{t}^{\prime}\right)^{*} \beta t_{0} t_{1} \ldots t_{n-1}\left(\dot{D}_{t}^{\prime}\right) \\
& =\gamma\left(\beta t_{n-1} t_{n-2} \ldots t_{1} t_{0} D_{t}^{\prime}\right)^{*}\left(\dot{D}_{t}^{\prime}\right) \quad \text { since }{ }^{*} \text { is an anti-involution } \\
& =\gamma z_{t}\left(D_{t}+Q\left(\dot{D}_{t}\right)+E\right)^{*}\left(\dot{D}_{t}^{\prime}\right) \quad \text { by }(6.8),
\end{aligned}
$$

where $E$ is a linear combination of diagrams with fewer than $t$ through strings. 
Now a straightforward computation shows that

$$
\left(\dot{D}_{t}^{*}\right)\left(\dot{D}_{t}^{\prime}\right)=\delta_{Q}\left(\dot{\mathrm{id}}_{t}\right) \quad \text { and } \quad D_{t}^{*}\left(\dot{D}_{t}^{\prime}\right)=\dot{\mathrm{id}}_{t} .
$$

Hence from (6.13.4) we have

$$
\begin{aligned}
\theta^{-}\left(\gamma \tau_{t}\right) & =\gamma z_{t}\left(D_{t}+Q\left(\dot{D}_{t}\right)+E\right)^{*}\left(\dot{D}_{t}^{\prime}\right) \\
& =z_{t} \gamma\left(\dot{\mathrm{id}}_{t}\right)\left(1+Q \delta_{Q}\right) \\
& =-Q^{2} z_{t} \gamma\left(\dot{\mathrm{id}}_{t}\right) \\
& =-Q^{2} z_{t} \dot{\gamma} .
\end{aligned}
$$

But $z_{t}^{2}=-q^{-t} Q^{-2}$, whence $q^{-t} z_{t}^{-1}=-Q^{2} z_{t}$, and (6.13.3) follows. This completes the proof of Theorem (6.13).

Notice that for any $t$, if $z_{t}=(-1)^{t} \beta Q^{-1} q^{-\frac{1}{2}(n+t-2)}$, then $z_{-t}=q^{t} z_{t}$. Hence in particular if $t \leqslant 0, q^{t} z_{-t}^{-1}=z_{t}^{-1}$. Hence Theorem (6.13) may be stated as follows.

(6.14) COROllary. - Suppose $t \in \mathcal{T}$ and $t \leqslant 0$. Then the inflation $g_{\beta}^{*} W_{t}(n) \cong W_{-t, z_{t}^{-1}}(n)$.

We may combine and generalise the statements of Theorems (6.1) and (6.13) as follows. Note that the condition on $\delta_{q}$ is absent from the statement below.

(6.15) Corollary. - Suppose $R$ is any commutative ring and suppose that $q, Q$ are elements of $R^{\times}$. Let $\beta \in R^{\times}$satisfy $\beta^{2}=-q^{n-2}$ and let $g_{\beta}: T_{n}^{a}(q) \rightarrow T L B_{n}(q, Q)$ be the surjection defined in (5.11). For $t \in \mathbb{Z}$ such that $|t| \leqslant n$ and $t \equiv n(\bmod 2)$, define $\varepsilon_{t}:=t /|t|$ for $t \neq 0$, and $\varepsilon_{t}=1$ if $t=0$.

Then the inflation $g_{\beta}^{*} W_{t}(n)$ of the cell module $W_{t}(n)$ of $T L B_{n}(q, Q)$ is isomorphic to the cell module $W_{|t|, z_{t}^{\varepsilon_{t}}}(n)$ of $T_{n}^{a}(q)$, where $z_{t}$ is as defined in the statement of (6.1).

Proof. - Replace $R$ in the statement of Theorems (6.1) or (6.13) by the ring $R\left[\mathbf{q}, \mathbf{q}^{-1}\right]$ of Laurent polynomials in the indeterminate $\mathbf{q}$ and replace $q \in R$ by $\mathbf{q} \in R\left[\mathbf{q}, \mathbf{q}^{-1}\right]$. Then all algebras and modules may be considered over $R\left[\mathbf{q}, \mathbf{q}^{-1}\right]$, and the hypotheses of Theorems (6.1) or (6.13) apply and we deduce that there is a (unique) isomorphism of $R\left[\mathbf{q}, \mathbf{q}^{-1}\right]$-modules:

$$
g_{\beta}^{*} W_{t}(n) \rightarrow W_{|t|, z_{t}^{\varepsilon}}(n)
$$

which takes a finite diagram in $W_{t}(n)$ to the same diagram in $W_{|t|, z_{t}^{\varepsilon_{t}}}(n)$. But $R$ is a module over $R\left[\mathbf{q}, \mathbf{q}^{-1}\right]$ via the homomorphism which takes $\mathbf{q}$ to $q$. Tensoring the above isomorphism with $R$ provides the required isomorphism.

\section{Eigenvalues of the translation elements $X_{i}$}

Recall that we have a 1-parameter family of surjections $\psi_{\alpha}: \widehat{H}_{n}^{a}(q) \rightarrow T_{n}^{a}(q)$ and that we wish to study the inflations $\psi_{\alpha}^{*} W_{t, z}(n)$. Now by Corollary (6.12), $W_{t, z}(n)$ may be realised as a pullback via $g_{\beta}=g_{\beta, Q}$ of some cell module $W_{t}(n)$ (for $t \geqslant 0$ ) of $T L B_{n}(q, Q)$. But by the commutativity of the diagram (5.12),

$$
\psi_{\alpha}^{*} W_{t, z}(n) \cong \psi_{\alpha}^{*} g_{\beta}^{*} W_{t}(n) \cong \xi_{Q, \mu}^{*} W_{t}(n),
$$

where $\alpha, \beta$ and $\mu$ are related by $\beta^{2}=-q^{n-2}$ and $\mu \alpha^{-1}=\beta$. 
Thus we study the structure of the pullbacks $\psi_{\alpha}^{*} W_{t, z}(n)$ by means of the pullbacks $\xi_{Q, \mu}^{*} W_{t}(n)$. For this we shall require some easy facts concerning the submodule structure of $W_{t}(n)$ on restriction to $T L B_{n-1}(q, Q)$. For $1 \leqslant i \leqslant n$, we regard $T L B_{i}(q, Q)$ as the subalgebra generated by $\left\{c_{0}, c_{1}, \ldots, c_{i-1}\right\}$; in terms of diagrams, it is the subalgebra spanned by diagrams with the rightmost $n-i$ top and bottom vertices joined by unmarked vertical through strings. Throughout this section we assume that $\delta_{q}$ is not a divisor of zero in $R$, since we shall require Theorems (6.1) and (6.13).

(7.2) Proposition. - Let $t \in \mathbb{Z}, 0 \leqslant|t| \leqslant n, n+t \in 2 \mathbb{Z}$.

(i) If $t \geqslant 0$, we have a short exact sequence

$$
0 \rightarrow W_{t-1}(n-1) \rightarrow \operatorname{Res}_{T L B_{n-1}(q, Q)}^{T L B_{n}(q, Q)} W_{t}(n) \rightarrow W_{t+1}(n-1) \rightarrow 0 .
$$

(ii) If $t<0$, we have a short exact sequence

$$
0 \rightarrow W_{t+1}(n-1) \rightarrow \operatorname{Res}_{T L B_{n-1}(q, Q)}^{T L B_{n}(q, Q)} W_{t}(n) \rightarrow W_{t-1}(n-1) \rightarrow 0 .
$$

Here we adopt the convention that $W_{j}(k)=0$ if $|j|>k$.

Proof. - (i) If $t>0$, the diagrams in $W_{t}(n)$ in which the rightmost top and bottom vertices are joined span a $T L B_{n-1}(q, Q)$-submodule which is clearly isomorphic to $W_{t-1}(n-1)$. The quotient module is spanned by the images of the other diagrams, which all have the property that the top right vertex is joined to another top vertex. Define a homomorphism $W_{t}(n) / W_{t-1}(n-1) \rightarrow W_{t+1}(n-1)$ by sending a basis diagram $\nu$ of $W_{t}(n) \backslash W_{t-1}(n-1)$ to the diagram $t+1 \rightarrow n-1$ obtained by moving the top right vertex to the bottom right without changing any arcs. One checks easily that this is an isomorphism of $T L B_{n-1}(q, Q)$-modules.

If $t=0$, the diagrams in $W_{t}(n)=W_{0}(n)$ such that the arc from the rightmost vertex is marked span a $T L B_{n-1}(q, Q)$-submodule which is clearly isomorphic to $W_{-1}(n-1)$ (imagine the rightmost vertex as the bottom vertex in a marked diagram $1 \rightarrow n-1$ ). The quotient $W_{0}(n) / W_{-1}(n-1)$ is spanned by the other diagrams, and by a similar argument is isomorphic as $T L B_{n-1}(q, Q)$-module to $W_{1}(n-1)$.

(ii) If $t<0$, the same construction as in (i) (but with care if $t=-1$, in which case we take the submodule spanned by diagrams with the rightmost top and bottom vertices joined by a marked string) provides a $W_{t-1}(n-1)$-submodule of $W_{t}(n)$ which is isomorphic to $W_{-(|t|-1)}(n-1)=W_{t+1}(n-1)$, with corresponding quotient $W_{t-1}(n-1)$.

(7.3) Corollary. - Let $t \in \mathbb{Z}, 0 \leqslant|t| \leqslant n, n+t \in 2 \mathbb{Z}$ and suppose $t \geqslant 0$. There is a filtration of $W_{t}(n)$ by $R$-submodules $W^{(i)}, i=n, n-1, n-2, \ldots, 1$, as in (7.3.1) below.

$$
\begin{aligned}
& W_{t}(n) \supset W_{t-1}(n-1) \supset \cdots \supset W_{0}(n-t) \supset W_{-1}(n-t-1) \supset W_{0}(n-t-2) \\
& \quad \supset W_{-1}(n-t-3) \supset \cdots \supset W_{0}(2) \supset W_{-1}(1) .
\end{aligned}
$$

Thus

$$
W^{(i)} \cong \begin{cases}W_{t-n+i}(i) & \text { if } n-t \leqslant i \leqslant n, \\ W_{0}(i) & \text { if } i \text { is even and } 0 \leqslant i \leqslant n-t, \\ W_{-1}(i) & \text { if } i \text { is odd and } 0 \leqslant i \leqslant n-t .\end{cases}
$$

For each $i=1,2,3, \ldots, n, W^{(i)}$ is a $T L B_{i}(q, Q)$-submodule of $W_{t}(n)$.

Proof. - This is immediate by repeated application of (7.2), which also gives an explicit description of the $W^{(i)}$ in terms of the diagrams of $W_{t}(n)$ which span them. For the reader's convenience, we give this description here. For $0 \leqslant i \leqslant n-t, W^{(i)}$ is the $R$-submodule spanned 
by diagrams with the rightmost $n-i$ top vertices joined to the rightmost bottom vertices by through strings. The "remaining" part of the diagram may then be thought of as an element of $W_{t-n+i}(i)$. In particular $W^{(n-t)} \cong W_{0}(n-t)$.

For $i=n-t-1$, we take $W^{(i)}$ to be the $R$ submodule spanned by diagrams in $W^{(n-t)}$ where the $(n-t)$ th top vertex is on a marked arc. By thinking of this $(n-t)$ th vertex as moved to the bottom, it is clear that $W^{(n-t-1)} \cong W_{-1}(n-t-1)$. The next term $W^{(n-t-2)}$ is spanned by diagrams in $W^{(n-t-1)}$ such that the top nodes $n-t$ and $n-t-1$ are joined (with a marked arc). Clearly $W^{(n-t-2)} \cong W_{0}(n-t-2)$, and we may now repeat the above construction until the sequence terminates.

To illustrate the construction in (7.3), observe that the diagram $E_{t}=E_{t, n}$ below lies in each of the submodules $W^{(i)}$.

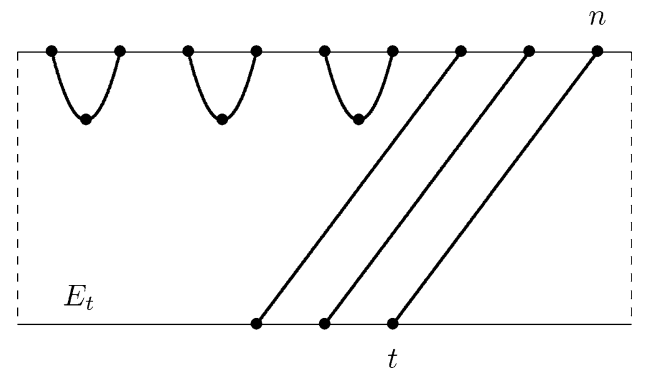

We shall now determine the eigenvalues of the translation elements $X_{i} \in \widehat{H_{n}^{a}}(q)$ on the inflation $\xi_{Q, \mu}^{*} W_{t}(n)$ (cf. (7.1)).

(7.4) Proposition. - The element $X_{1} X_{2} \ldots X_{n} \in \widehat{H_{n}^{a}}(q)$ acts on the module

$$
M=\xi_{Q, \mu}^{*} W_{t}(n)
$$

as multiplication by the scalar

$$
h(n, t)=(-1)^{\frac{n+t}{2}} \mu^{n} Q^{-t} q^{\frac{1}{2}\left(2 n-n^{2}-t^{2}\right)} .
$$

Proof. - First take $t \geqslant 0$. By (7.1), $\xi_{Q, \mu}^{*} W_{t}(n) \cong \psi_{\alpha}^{*} W_{t, z}(n)$, where $z=z_{t}$. But by (3.6.1), $X_{1} X_{2} \ldots X_{n}=V^{n}$, and by (5.13), $\psi_{\alpha}\left(V^{n}\right)=\alpha^{n} \tau^{n} \in T_{n}^{a}(q)$. Moreover $\tau^{n}$ is central in $T_{n}^{a}(q)$ and acts on $W_{t, z}(n)$ as multiplication by $z^{t}$. Hence $X_{1} X_{2} \ldots X_{n}$ acts on $\xi_{Q, \mu}^{*} W_{t}(n)$ as multiplication by the scalar

$$
\alpha^{n} z_{t}^{t}=\alpha^{n}\left((-1)^{t} \beta Q^{-1} q^{-\frac{1}{2}(n+t-2)}\right)^{t},
$$

which after simplification using the relations $\beta^{2}=-q^{n-2}$ and $\beta=\alpha^{-1} \mu$ is easily shown to be equal to $h(n, t)$.

If $t<0$, the proof is the same, but we use Theorem (6.13) instead of (6.1).

For the remainder of this section, we take $t \geqslant 0$.

The inflation $\xi_{Q, \mu}^{*} W_{t}(n)$ defines a structure of $\widehat{H_{n}^{a}}(q)$-module on $W_{t}(n)$, and for $i=1,2, \ldots, n$, the submodule $W^{(i)}$ of the filtration (7.3.1) is stable under the action of the subalgebra $T L B_{i}(q, Q)$ which is the image under $\xi_{\mu, Q}$ of the subalgebra $\widehat{H_{i}^{a}}(q)$ of $\widehat{H_{n}^{a}}(q)$ which is generated by $\left\{X_{1}, T_{1}, \ldots, T_{i-1}\right\}$. We shall refer to the submodules $W^{(i)}$ as $\widehat{H_{i}^{a}}(q)$-modules, with the understanding that the action is induced by $\xi_{\mu, Q}$. Now $\widehat{H_{i}^{a}}(q)$ contains the elements $\left\{X_{1}, X_{2}, \ldots, X_{i}\right\}$ and it follows from (7.4) that 
(7.5) Corollary. - The element $X_{1} X_{2} \ldots X_{i} \in \widehat{H_{n}^{a}}(q)$ acts on the submodule $W^{(i)}$ of $\xi_{Q, \mu}^{*} W_{t}(n)$ as multiplication by the scalar $\beta_{i}$, where

$$
\beta_{i}= \begin{cases}(-1)^{\frac{t-n+2 i}{2}} \mu^{i} Q^{n-t-i} q^{\frac{1}{2}\left(2 i-i^{2}-(t-n+i)^{2}\right)} & \text { if } n-t \leqslant i \leqslant n, \\ (-1)^{\frac{i}{2}} \mu^{i} q^{\frac{1}{2}\left(2 i-i^{2}\right)} & \text { if } i \text { is even and } 1 \leqslant i \leqslant n-t, \\ (-1)^{\frac{i-1}{2}} \mu^{i} Q q^{\frac{1}{2}\left(2 i-i^{2}-1\right)} & \text { if } i \text { is odd and } 1 \leqslant i \leqslant n-t .\end{cases}
$$

Proof. - Using the identification of $W^{(i)}$ as a $T L B_{i}(q, Q)$-module given in (7.3), it follows from (7.4) that $X_{1} X_{2} \ldots X_{i}$ acts as a scalar $\beta_{i}$ on $W^{(i)}$, and that in the three cases $n-t \leqslant i \leqslant n$, $1 \leqslant i \leqslant n-t$ and $i$ even and $1 \leqslant i \leqslant n-t$ and $i$ odd, the value of $\beta_{i}$ is respectively given by $h(i, t-n+i), h(i, 0)$ and $h(i,-1)$. The formulae in the statement are the result.

We shall determine the action of the $X_{i}$ using the fact that

$$
X_{i}=\left(X_{1} X_{2} \ldots X_{i}\right)\left(X_{1} X_{2} \ldots X_{i-1}\right)^{-1}
$$

(7.6) COROLLARY. - In the filtration of (7.3)

$$
W_{t}(n)=W^{(n)} \supset \cdots \supset W^{(i)} \supset W^{(i-1)} \supset \cdots \supset W^{(1)} \supset 0,
$$

where $W^{(i)}$ is thought of as a $\widehat{H_{i}^{a}}(q)$-module, we have, for any pair of integers $i, j$ such that $1 \leqslant j<i \leqslant n$ that $X_{i}$ acts on $W^{(j)}$ as multiplication by $\gamma_{i}$, where $\gamma_{i}$ is given by

$$
\gamma_{i}= \begin{cases}-\mu Q^{-1} q^{n+2-t-2 i} & \text { if } n-t \leqslant i \leqslant n, \\ -\mu Q^{-1} q^{2-i} & \text { if } i \text { is even and } 1 \leqslant i \leqslant n-t, \\ \mu Q q^{1-i} & \text { if } i \text { is odd and } 1 \leqslant i \leqslant n-t .\end{cases}
$$

Proof. - Since $W^{(j)}$ is a submodule of $W^{(i-1)}$, it will suffice to show that $X_{i}$ acts on $W^{(i-1)}$ as multiplication by $\gamma_{i}$. But $W^{(i-1)}$ is a submodule of $W^{(i)}$, and hence by (7.5), the elements $Y_{i}=X_{1} X_{2} \ldots X_{i}$ and $Y_{i-1}=X_{1} X_{2} \ldots X_{i-1}$ act as the scalars $\beta_{i}, \beta_{i-1}$ respectively on $W^{(i-1)}$ (here we take $Y_{0}=1$ ). It follows that $X_{i}=Y_{i} Y_{i-1}^{-1}$ acts on $W^{(i-1)}$ as the scalar $\gamma_{i}=\beta_{i} \beta_{i-1}^{-1}$, where $\beta_{0}=1$. An easy calculation now completes the proof.

(7.7) Proposition. - There is an ordering of the diagrams which form an R-basis of $W_{t}(n)$ which is compatible with the filtration (7.3) and with respect to which each element $X_{i}$ $(i=1,2, \ldots, n)$ has upper triangular matrix.

Proof. - We use induction on $n$; for $n=1$ the result is trivial. Assume the result true for $W_{t-1}(n-1)$ and $W_{t+1}(n-1)$. Then there is an ordering of the diagrams in $W^{(n)}$ which is compatible with the filtration $W^{(n)} \supset W^{(n-1)} \supset \cdots \supset 0$ such that $X_{1}, \ldots, X_{n-1}$ act in upper triangular fashion. This is because by induction we have such an ordering of the diagrams in $W^{(n-1)}$, while $W^{(n)} / W^{(n-1)} \cong W_{t-1}(n-1)$ or $W^{(n)} / W^{(n-1)} \cong W_{t+1}(n-1)$, whence again by induction, there is an appropriate ordering of the other diagrams in $W_{t}(n)$. But by (7.4), $Y_{n}:=X_{1} X_{2} \ldots X_{n}$ acts as a scalar on $W_{t}(n)$, whence $X_{n}=Y_{n} X_{1}^{-1} X_{2}^{-1} \ldots X_{n-1}^{-1}$ is also upper triangular.

(7.8) Definition. - (i) We denote by $U(n)$ the subalgebra of $\widehat{H_{n}^{a}}(q)$ generated by $\left\{X_{1}, \ldots, X_{n}\right\}$. By (3.3) this is isomorphic to the ring $R\left[X_{1}^{ \pm 1}, \ldots, X_{n}^{ \pm 1}\right]$ of Laurent polynomials in the $X_{i}$. 
(ii) A character $\chi: U(n) \rightarrow R^{\times}$is the linear extension to $U(n)$ of a multiplicative homomorphism $\chi: U(n)^{\times} \rightarrow R^{\times}$, where $U(n)^{\times}$is the group generated by the $X_{i}, i=1, \ldots, n$ (which is isomorphic to $\mathbb{Z}^{n}$ ). Such a character is clearly determined by the images of the $X_{i}$ and hence the group of characters may be identified with $\left(R^{\times}\right)^{n}$.

(iii) If $\chi: U(n) \rightarrow R^{\times}$is a character, write $\sigma(\chi)=\left(\chi\left(X_{1}\right), \ldots, \chi\left(X_{n}\right)\right) \in\left(R^{\times}\right)^{n}$ and $|\sigma(\chi)|$ for the multiset (i.e. set with multiplicities) $\left\{\chi\left(X_{1}\right), \ldots, \chi\left(X_{n}\right)\right\}$. We sometimes identify $\sigma(\chi)$ with $\chi$ and refer to the sequence $\sigma(\chi)$ as a character.

Now any diagram $D \in W_{t}(n)$ may be considered as an element of an ordered basis of $W_{t}(n)$ as in (7.7). Hence $D$ defines a character $\chi_{D}$ of $U(n)$ by

$$
X_{i} D \in \chi_{D}\left(X_{i}\right) D+\sum_{E \in W_{t}(n), E \neq D} R E
$$

where the sum is over diagrams in $W_{t}(n)$ which are distinct from (and ordered lower than) $D$.

(7.10) THEOREM. - Assume $t \geqslant 0$. For any diagram $D \in W_{t}(n)$, the character $\chi_{D}$ satisfies $\left|\sigma\left(\chi_{D}\right)\right|=\left\{\gamma_{1}, \gamma_{2}, \ldots, \gamma_{n}\right\}$, where the $\gamma_{i}$ are as in (7.6), i.e.,

$$
\begin{aligned}
\left\{\gamma_{1}, \ldots, \gamma_{n}\right\}= & \left\{\mu Q, \mu Q q^{-2}, \mu Q q^{-4}, \ldots, \mu Q q^{-(n-t-2)}\right\} \\
& \cup\left\{-\mu Q^{-1},-\mu Q^{-1} q^{-2},-\mu Q^{-1} q^{-4}, \ldots,-\mu Q^{-1} q^{-(n+t-2)}\right\},
\end{aligned}
$$

where the union is in the sense of multisets.

Proof. - This will be by induction on $n$. For $n=1$ the result is trivial. Let $D$ be any diagram in $W^{(n)}$. Then there is a unique index $i$ such that $D \in W^{(i)} \backslash W^{(i-1)}$, where $\ldots W^{(i)} \supset W^{(i-1)} \ldots$ is the filtration (7.3) of $W_{t}(n)$. If $i<n$, it follows by induction on $n$ that $\left\{\chi_{D}\left(X_{1}\right), \ldots, \chi_{D}\left(X_{i}\right)\right\}=\left\{\gamma_{j}=\beta_{j} \beta_{j-1}^{-1} \mid 1 \leqslant j \leqslant i\right\}$. Moreover for $j>i$, it follows from (7.6) that $X_{j} D=\gamma_{j} D$, since $X_{j}$ acts as $\gamma_{j}$ id on $W^{(i)}$. Thus the assertion (7.10) is true if $i<n$. Hence we consider the case $i=n$.

If $t \neq n$, then by (7.2.1), $W^{(n)} / W^{(n-1)} \cong W_{t+1}(n-1)$ as $T L B_{n-1}(q, Q)$-module, and hence

$$
X_{1} X_{2} \ldots X_{n-1} D \equiv h(n-1, t+1) D\left(\bmod W^{(n-1)}\right) .
$$

Hence $\chi_{D}\left(X_{n}\right)=h(n, t) h(n-1, t+1)^{-1}=\mu Q q^{-(n-t-2)}$. The set $\left\{\chi_{D}\left(X_{i}\right) \mid 1 \leqslant i \leqslant n-1\right\}$ may be determined by thinking of the $X_{i}, 1 \leqslant i \leqslant n-1$ as acting on the image of $D$ in $W^{(n)} / W^{(n-1)} \cong W_{t+1}(n-1)$. Then by induction we see that $\left\{\chi_{D}\left(X_{i}\right) \mid 1 \leqslant i \leqslant n-1\right\}$ is the set in the statement of $(7.1)$, with $(n, t)$ replaced by $(n-1, t+1)$, i.e.

$$
\left\{\chi_{D}\left(X_{i}\right) \mid 1 \leqslant i \leqslant n-1\right\}=\left\{\gamma_{1}, \ldots, \gamma_{n}\right\} \backslash\left\{\mu Q q^{-(n-t-2)}\right\},
$$

and hence $\left\{\chi_{D}\left(X_{i}\right) \mid 1 \leqslant i \leqslant n-1\right\}$ is as stated in (7.1). This proves (7.10) for the case $t \neq n$.

If $t=n, W_{t}(n) \cong W_{t-1}(n-1)$ as $T L B_{n-1}(q, Q)$-module, whence

$$
\left\{\chi_{D}\left(X_{i}\right) \mid 1 \leqslant i \leqslant n-1\right\}=\left\{\gamma_{1}, \ldots, \gamma_{n}\right\} \backslash\left\{-\mu Q^{-1} q^{-(2 n-2)}\right\} .
$$

But $X_{1} X_{2} \ldots X_{n} \in \widehat{H_{n}^{a}}(q)$ acts on the module $W_{n}(n)$ as multiplication by the scalar

$$
h(n, n)=(-1)^{n} \mu^{n} Q^{-n} q^{\left(n-n^{2}\right)} .
$$

Hence $X_{n}=-\mu Q^{-1} q^{-(2 n-2)}$ id on $W_{n}(n)$, which completes the proof for the case $t=n$. 


\section{The case of generic parameters}

In this section we prove that the inflations $\psi_{\alpha}^{*} W_{t, z}(n) \cong \xi_{Q, \mu}^{*} W_{t}(n)$ are representations of $\widehat{H_{n}^{a}}(q)$ which are induced from parabolic subalgebras, provided that the pair of parameters $q, Q$ is "generic". First we make precise the meaning of this term.

(8.1) Definition. - We say that the pair $(q, Q)$ of invertible elements is generic in the integral domain $R$ if $q$ is not a root of unity and there is no solution $m \in \mathbb{Z}$ for the equation $q^{m}= \pm Q^{2}$.

(8.2) Proposition. - Suppose that the pair $(q, Q)$ is generic.

(i) The elements of the multiset $\left\{\gamma_{1}, \ldots, \gamma_{n}\right\}$ of (7.10) are distinct, i.e. have multiplicity one. Moreover if $\chi_{D}\left(X_{i}\right)=\chi_{D}\left(X_{j}\right) q^{-2}$, then $j<i$.

(ii) The characters $\chi_{D}$ are distinct; i.e. if $\chi_{D_{1}}=\chi_{D_{2}}$ for diagrams $D_{1}, D_{2} \in W_{t}(n)$, then $D_{1}=D_{2}$.

Proof. - Observe first that by (7.6), the sequence $\left(\gamma_{1}, \ldots, \gamma_{n}\right)$ is given by

$$
\begin{aligned}
& \left(\mu Q,-\mu Q^{-1}, \mu Q q^{-2},-\mu Q^{-1} q^{-2}, \ldots, \mu Q q^{-(n-t-2)},-\mu Q^{-1} q^{-(n-t-2)},\right. \\
& \left.-\mu Q^{-1} q^{-(n-t)},-\mu Q^{-1} q^{-(n-t+2)}, \ldots,-\mu Q^{-1} q^{-(n+t-2)}\right) .
\end{aligned}
$$

The first assertion of (i) is clear. For the second assertion, we use the argument in the proof of (7.10). If $D \in W^{(i)} \backslash W^{(i-1)}$, then

$$
\sigma\left(\chi_{D}\right)=\left(\chi_{D}^{(i)}\left(X_{1}\right), \chi_{D}^{(i)}\left(X_{2}\right), \ldots, \chi_{D}^{(i)}\left(X_{i}\right), \gamma_{i+1}, \ldots, \gamma_{n}\right)
$$

where $\chi_{D}^{(i)}$ is the character of $U(i)=R\left[X_{1}^{ \pm 1}, \ldots, X_{i}^{ \pm 1}\right]$ on $W^{(i)}$ which corresponds to $D \in$ $W^{(i)}$. Inspection of the sequence (8.2.1) (and induction on $n$ ) now yields the second assertion if $i<n$.

If $i=n$, the argument of (i) shows that

$$
\sigma\left(\chi_{D}\right)=\left(\chi_{D}^{\prime}\left(X_{1}\right), \chi_{D}^{\prime}\left(X_{2}\right), \ldots, \chi_{D}^{\prime}\left(X_{n-1}\right), \mu Q q^{-(n-t-2)}\right)
$$

where $\chi_{D}^{\prime}$ is the character of $U(n-1)$ corresponding to $D \in W^{(n)} / W^{(n-1)} \cong W_{t+1}(n-1)$. Again by induction, the assertion is true for this sequence, whence the result. This proves (i).

The proof of (ii) is also by induction on $n$. The result is trivial for $n=1$. Let $D_{1}, D_{2}$ be distinct diagrams in $W=W^{(n)}$. If $D_{1}, D_{2}$ are either both in $W^{(n-1)}$ or both in $W^{(n)} \backslash W^{(n-1)}$, the result is immediate by induction. Thus we may take $D_{1} \in W^{(n-1)}$ and $D_{2} \in W^{(n)} \backslash W^{(n-1)}$. But then $\chi_{D_{1}}\left(X_{1} \ldots X_{n-1}\right) \neq \chi_{D_{2}}\left(X_{1} \ldots X_{n-1}\right)$ by Theorem (7.4), since in the generic case $h(n-1, t-1) \neq h(n-1, t+1)$. This completes the proof of (8.2).

(8.3) Corollary. - Assume that the pair $(q, Q)$ is generic (see (8.1)). Define sequences $\Gamma_{1}, \Gamma_{2}$ as in (8.3.1) below.

$$
\begin{aligned}
& \Gamma_{1}=\left(\mu Q, \mu Q q^{-2}, \mu Q q^{-4}, \ldots, \mu Q q^{-(n-t-2)}\right) \\
& \Gamma_{2}=\left(-\mu Q^{-1},-\mu Q^{-1} q^{-2},-\mu Q^{-1} q^{-4}, \ldots,-\mu Q^{-1} q^{-(n+t-2)}\right) .
\end{aligned}
$$

Then $\Gamma_{1}, \Gamma_{2}$ are disjoint and have cardinalities $k=\frac{n-t}{2}, n-k$ respectively. The set of characters (cf. Definition (7.8)(iii)) $\left\{\sigma\left(\chi_{D}\right) \mid D\right.$ a diagram in $\left.W_{t}(n)\right\}$ coincides with the set of all orderings of $\Gamma:=\Gamma_{1} \amalg \Gamma_{2}$ in which $\Gamma_{1}, \Gamma_{2}$ appear in the given order. 
Proof. - By (7.10), each character $\chi_{D}$ corresponds to some ordering of $\Gamma$. In the generic case, the elements of $\Gamma$ are distinct, whence the corresponding ordering is unique. But by the second assertion in Proposition (8.2)(i), in any ordering corresponding to a character $\chi_{D}, \Gamma_{1}$ and $\Gamma_{2}$ appear in the given order. But the number of such orderings of $\Gamma$ is $\left(\begin{array}{c}n \\ \frac{n-t}{2}\end{array}\right)$, which coincides with the number of distinct diagrams $D \in W_{t}(n)$ (see the paragraph preceding (6.1)). The assertion follows.

(8.4) Corollary. - Suppose that $R$ is any integral domain and let $(q, Q)$ be generic in $R$. There is a non-zero element $\Delta \in R$ with the following property. Let $R_{\Delta}$ be the localisation $R_{\Delta}=R\left[\Delta^{-1}\right]$. Write $M$ for the $\widehat{H_{n}^{a}}(q)$-module $\xi_{Q, \mu}^{*} W_{t}(n)$ and let $M_{\Delta}:=R_{\Delta} \otimes_{R} M$. Then $M_{\Delta}$ has a unique decomposition $M_{\Delta} \cong \bigoplus_{D} M_{\Delta}(D)$, where the sum is over the diagrams in $W_{t}(n)$ and $M_{\Delta}(D)$ is a free $R_{\Delta}$-submodule of rank one, which is invariant under $U(n)_{\Delta}=$ $R_{\Delta}\left[X_{1}^{ \pm 1}, \ldots, X_{n}^{ \pm 1}\right]$, and on which $U(n)_{\Delta}$ acts via the character $\chi_{D}$. Any simultaneous eigenvector $E \in M_{\Delta}$ for the $X_{i}$ corresponds to one of the characters $\chi_{D}$ of $U(n)$.

Proof. - By the first statement in (8.2)(i), the characters $\chi_{D}$ are distinct. Hence there is an element $Y \in U(n)$ whose set $\left\{\chi_{D}(Y) \mid D\right.$ a diagram in $\left.W_{t}(n)\right\}$ of eigenvalues (recall that the elements of $U(n)$ act in triangular fashion on $W_{t, z}(n)$ and the $\chi_{D}(Y)$ are the diagonal elements) is distinct. It is easy to show using elementary linear algebra that there is a set of elements $x_{D} \in M$ such that $Y x_{D}=\chi_{D}(Y)$ (for each diagram $D$ ). If $\Delta$ is the (non-zero) determinant of the matrix formed by the coefficients of the $x_{D}$ with respect to an $R$-basis of $M$, it is clear that $M_{\Delta}$ is the direct sum of the rank one submodules $R_{\Delta} x_{D}$, which are each invariant under $Y$. Since $Y$ has distinct eigenvalues, any linear transformation of $M_{\Delta}$ which commutes with $Y$ is diagonal with respect to this eigenspace decomposition, whence we deduce that the eigenspaces of $Y$ are invariant under $U(n)$.

Finally, if $E$ is a simultaneous eigenvector of the $X_{i}$, it is an eigenvector of $Y$, whence $E \in M_{\Delta} x_{D}$ for some diagram $D$. This proves the last statement.

Note that if $R$ is a field in (8.4), the statement may be simplified by eliminating any reference to $\Delta$.

We shall refer to an ordering of $\Gamma$ satisfying the condition of (8.3) as permissible. Note that one permissible ordering is

$$
(\Gamma)=\left(-\mu Q^{-1},-\mu Q^{-1} q^{-2}, \ldots,-\mu Q^{-1} q^{-(n+t-2)}, \mu Q, \mu Q q^{-2}, \ldots, \mu Q q^{-(n-t-2)}\right) .
$$

We write $\left(\Gamma_{2}, \Gamma_{1}\right)=\left(\delta_{1}, \ldots, \delta_{n}\right)$ for this ordering.

(8.5) Corollary. - With notation as in (8.3), let $R$ be a field. Then there is a vector $E \in M$ which is a simultaneous eigenvector of $X_{1}, X_{2}, \ldots, X_{n}$ such that $\chi_{E}=\left(\delta_{1}, \ldots, \delta_{n}\right)$.

This is clear from (8.4).

We shall require the following elementary result from linear algebra.

(8.6) Lemma. - Let $\mathcal{V}$ be a vector space over a field $R$. Let $A, B$ be commuting linear transformations of $\mathcal{V}$ and let $v \in \mathcal{V}$ satisfy

$$
(A+B) v=(a+b) v \quad \text { and } \quad A B v=a b v \quad \text { for some } a, b \in R .
$$

Assume that $A v \notin R v$. Then

(i) The plane $\Pi=\langle v, A v\rangle=\langle v, B v\rangle$ is stable under the semigroup generated by $A, B$.

(ii) The eigenvalues of $A, B$ on $\Pi$ are each $\{a, b\}$.

(iii) If $v_{1}=b v-A v, v_{2}=a v-A v$ then $A v_{1}=a v_{1}, B v_{1}=b v_{1}, A v_{2}=b v_{2}$ and $B v_{2}=a v_{2}$. 
The proof is easy.

(8.7) Proposition. - Let $E \in M$ be the eigenvector of (8.5). Then $T_{j} E=-q^{-1} E$ for $j \neq \frac{n+t}{2}, j=1,2, \ldots, n-1$.

Proof. - Take $j \in \mathbb{Z}, 1 \leqslant j \leqslant n-1, j \neq \frac{n+t}{2}$, and let $v=T_{j} E$. Then since $X_{i}$ commutes with $T_{j}$ unless $i=j$ or $i=j+1$ (see (2.6.1) or Proposition (3.3)(iv)), we have

$$
X_{i} v=\delta_{i} v \quad \text { if } i \neq j, j+1 .
$$

Moreover since $T_{j}$ commutes with $X_{j} X_{j+1}$ and $X_{j}+X_{j+1}$ (by the Bernstein relation (3.3)(iv)), we have $X_{j} X_{j+1} v=\delta_{j} \delta_{j+1} v$ and $\left(X_{j}+X_{j+1}\right) v=\left(\delta_{j}+\delta_{j+1}\right) v$.

Observe that $X_{j} v=X_{j} T_{j} E=T_{j}^{-1} X_{j+1} E=\delta_{j+1} T_{j}^{-1} E$. But since $T_{j}^{-1}=T_{j}+q^{-1}-q$, we have

$$
X_{j} v=\delta_{j+1}\left(T_{j} E+\left(q^{-1}-q\right) E\right)=\delta_{j+1}\left(v+\left(q^{-1}-q\right) E\right) .
$$

Suppose $X_{j} v$ is not a scalar multiple of $v$. Then we may apply (8.6) with $A=X_{j}, B=X_{j+1}$, $a=\delta_{j}$ and $b=\delta_{j+1}$. By (8.6) the vector $v_{2}=\delta_{j} v-X_{j} v$ is a simultaneous eigenvector for $X_{j}, X_{j+1}$ with respective eigenvalues $\delta_{j+1}, \delta_{j}$. Moreover by (8.7.1), $v_{2}$ is also an eigenvector for each $X_{i}(i \neq j, j+1)$ with corresponding eigenvalue $\delta_{i}$. Thus $v_{2}$ corresponds to some character $\chi_{D}$ of $U(n)$ by the last statement in (8.4). But our choice of $j$ ensures that $\delta_{j+1}=\delta_{j} q^{-2}$, which by the second statement in (8.2)(i) is impossible. It follows that $X_{j} v \in R v$. Hence by (8.7.2), $T_{j} E \in R E$. By the quadratic relation for $T_{j}$, we therefore have $T_{j} E=q E$ or $T_{j} E=-q^{-1} E$; but $X_{j+1} E=T_{j} X_{j} T_{j} E=q^{-2} X_{j} E$, whence $T_{j} E=-q^{-1} E$ as stated.

\section{Induced modules and standard modules}

We shall henceforth assume that $R$ is a field and all modules and algebras will be $R$-modules and $R$-algebras.

We shall identify $M=\xi_{Q, \mu}^{*} W_{t}(n)$ as a module induced from a one-dimensional module of a parabolic subalgebra of $\widehat{H_{n}^{a}}(q)$. Such modules were introduced by Rogawski [28]. The parabolic subalgebras are defined as follows. For any subset $J \subset\{1,2, \ldots, n-1\}$ let $\widehat{H_{J}^{a}}(q)$ be the subalgebra of $\widehat{H_{n}^{a}}(q)$ which is generated by $U(n)=R\left[X_{1}^{ \pm 1}, \ldots, X_{n}^{ \pm 1}\right]$ and $\left\{T_{j} \mid j \in J\right\}$. For any partition $\lambda=\lambda_{1} \geqslant \lambda_{2} \geqslant \cdots \geqslant \lambda_{\ell} \geqslant 0$ of $n$, define $\widehat{H_{\lambda}^{a}}(q)$ to be $\widehat{H_{J}^{a}}(q)$, where $J=J_{\lambda}$, defined as follows: $j \in J_{\lambda} \Leftrightarrow \exists k \in\{1,2, \ldots, \ell\}$ with $n \geqslant \sum_{i=1}^{k} \lambda_{i} \geqslant j+1>j>\sum_{i=1}^{k-1} \lambda_{i}$, where, if $k=0$, the smaller sum is interpreted as 0 . The subalgebra $\widehat{H_{\lambda}^{a}}(q)$ has finite index equal to the multinomial coefficient $\frac{n !}{\lambda_{1} ! \ldots \lambda_{\ell} !}$ in $\widehat{H_{n}^{a}}(q)$.

Now let $R_{\lambda, \chi}$ be a one-dimensional $\widehat{H_{\lambda}^{a}}(q)$-module on which $T_{j}$ acts as multiplication by $q$ and $U(n)=R\left[X_{1}^{ \pm 1}, \ldots, X_{n}^{ \pm 1}\right]$ acts via the character $\chi$. Then since $X_{j+1}=T_{j} X_{j} T_{j}$

$$
\chi\left(X_{j+1}\right)=q^{2} \chi\left(X_{j}\right) \quad \text { for each } j \in J_{\lambda},
$$

and any character satisfying this condition gives rise to a one-dimensional $\widehat{H_{\lambda}^{a}}(q)$-module. A similar definition applies when $J_{\lambda}$ is replaced by an arbitrary subset $J$ of $\{1, \ldots, n-1\}$.

(9.2) Definition. - Let $\chi$ be a character of $U(n)=R\left[X_{1}^{ \pm 1}, \ldots, X_{n}^{ \pm 1}\right]$ which satisfies condition (9.1). Define the $\widehat{H_{n}^{a}}(q)$-module $K_{\lambda, \chi}$ as the induced module

$$
K_{\lambda, \chi}=\widehat{H_{n}^{a}}(q) \otimes_{\widehat{H_{\lambda}^{a}}(q)} R_{\lambda, \chi}
$$


where $R_{\lambda, \chi}$ is the one-dimensional $\widehat{H_{\lambda}^{a}}(q)$-module defined above. Then the dimension of $K_{\lambda, \chi}$ is $\frac{n !}{\lambda_{1} ! \ldots \lambda_{\ell} !}$.

Of course this definition applies slightly more generally to the subalgebras $\widehat{H_{J}^{a}}(q)$, where $J_{\lambda}$ is replaced by an arbitrary subset $J \subset\{1,2, \ldots, n-1\}$ and the corresponding module is denoted by $K_{J, \chi}$.

We have the following easy characterisation of these induced modules.

(9.3) LEMMA. - Suppose $K$ is a finite dimensional $\widehat{H_{n}^{a}}(q)$-module which contains a vector $e$ which generates $K$ as $\widehat{H_{n}^{a}}(q)$-module and satisfies the following two conditions for some subset $J \subset\{1,2, \ldots, n-1\}:$

$$
T_{i} e=q e \quad \text { for } i \in J
$$

and

$$
X_{i} e=\chi\left(X_{i}\right) e, \quad 1 \leqslant i \leqslant n, \text { for some character } \chi \text { of } U(n) .
$$

Then $K$ is a quotient of $K_{J, \chi}$. In particular, if $\operatorname{dim}(K)=\operatorname{dim}\left(K_{J, \chi}\right)$, we have equality.

We wish to identify these modules as "standard modules" in the sense of Kazhdan and Lusztig. Write $G=G L_{n}(R)$ and $\mathfrak{G}=\operatorname{Lie}(G)$. Then $U(n)=R\left[X_{1}^{ \pm 1}, \ldots, X_{n}^{ \pm 1}\right]$ may be thought of as the coordinate ring of the maximal torus $S$ of $G$ consisting of diagonal elements. Any character $\chi$ of $U(n)=R\left[X_{1}^{ \pm 1}, \ldots, X_{n}^{ \pm 1}\right]$ is therefore given by evaluation at a (generically) unique diagonal element $s \in S$. It is not difficult to see that in fact the set of pairs $(\lambda, \chi)$, where $\chi$ satisfies condition (9.1), modulo the equivalence defined by isomorphism of the corresponding induced representations $K_{\lambda, \chi}$, is thus in bijection with the set $\widehat{\mathcal{P}}$ of equivalence classes of pairs $(s, N)$ modulo $G$, where $s \in G$ is semisimple, $N \in \mathfrak{G}$ is nilpotent and $s . N=q^{2} N$ (the action of $G$ on $\mathfrak{G}$ being the adjoint representation). The correspondence may be realised as follows. Let $J_{k}$ denote the $k \times k$ (Jordan) matrix with zeros everywhere except on the super-diagonal, where all entries are 1. Given a partition $\lambda=\lambda_{1} \geqslant \cdots \geqslant \lambda_{\ell}>0$ of $n$, there is a unique block diagonal matrix $N_{\lambda}$ whose diagonal (Jordan) blocks have size $\lambda_{i}, i=1,2, \ldots, \ell$. If $R$ is algebraically closed, then each pair $(s, N)$ such that $\operatorname{Ad}(g) \cdot N=q^{2} N$ is $G$-conjugate to one where

$$
N=N_{\lambda}=\left[\begin{array}{ccc}
J_{\lambda_{1}} & & \cdots \\
& J_{\lambda_{2}} & \cdots \\
& \cdots & \\
& \cdots & J_{\lambda_{\ell}}
\end{array}\right]
$$

and $s$ is also block diagonal, of the form

$$
s=s_{\lambda}=\left[\begin{array}{ccc}
s_{\lambda_{1}} & & \cdots \\
& s_{\lambda_{2}} & \cdots \\
& \cdots & \\
& \cdots & s_{\lambda_{\ell}}
\end{array}\right]
$$

where the matrices $s_{\lambda_{i}}$ are diagonal of the form

$$
s_{\lambda_{i}}=\left[\begin{array}{lllll}
a_{i} & & & & \\
& a_{i} q^{-2} & & & \\
& & a_{i} q^{-4} & & \\
& & & \cdot & \\
& & & \cdot & \\
& & & & a_{i} q^{-2\left(\lambda_{i}-1\right)}
\end{array}\right] .
$$


The matrix $s_{\lambda}$ depends on the $\ell$ parameters $a_{i} \in R^{\times}$, and the two pairs $\left(s_{\lambda}, N_{\lambda}\right)$ and $\left(s_{\lambda^{\prime}}^{\prime}, N_{\lambda^{\prime}}\right)$ are $G$-conjugate if and only if $\lambda=\lambda^{\prime}$ and some parts of $\lambda$ are equal and the corresponding $s_{\lambda_{i}}$ are permuted to obtain $s^{\prime}$.

In view of the correspondence between pairs $(s, N) \in \widehat{\mathcal{P}}$ and pairs $(\lambda, \chi)$ as in (9.2) we shall use the notation

$$
K_{\lambda, \chi}=K_{s, N}
$$

The character $\chi$ is given in terms of the pair $(s, N)$ by $\chi\left(X_{i}\right)=s_{i}^{-1}$, where $s_{i}$ is the $i$ th diagonal entry in $s_{i}$.

Whenever we use the notation $(s, N)$, we shall assume that $R=\mathbb{C}$ unless we specify otherwise, since we aim to integrate our results with the body of literature which relates to this case. Now Chriss and Ginzburg [5] and Kazhdan and Lusztig [19], (see [30] for an exposition) have defined "standard modules" $M_{s, N}$ for $\widehat{H_{n}^{a}}(q)$ (of course as a special case of a general construction) in the case $R=\mathbb{C}$. The space of $M_{s, N}$ is the Borel-Moore homology $H_{*}\left(\mathcal{B}_{N}^{s}\right)$ of a certain subvariety $\mathcal{B}_{N}^{s}$ of the flag variety of $G$. We shall need the following result of Ariki. For any finite dimensional $\widehat{H_{n}^{a}}(q)$-module $M,[M]$ denotes its class in the Grothendieck group of finite dimensional $\widehat{H_{n}^{a}}(q)$-modules.

(9.5) Proposition [1, Theorem 3.2, p. 798]. - We have, in the Grothendieck group of finite dimensional $\widehat{H_{n}^{a}}(q)$-modules,

$$
\left[M_{s, N}\right]=\left[K_{s, N}\right]=\left[K_{\lambda, \chi}\right]=\left[\widehat{H_{n}^{a}}(q) \otimes \widehat{H_{\lambda}^{a}(q)} \mathbb{C}_{\lambda, \chi}\right]
$$

for each pair $(s, N) \in \widehat{\mathcal{P}}$, where $N=N_{\lambda}$ and $\chi\left(X_{i}\right)=s_{i}^{-1}$ if $s=\operatorname{diag}\left(s_{1}, \ldots, s_{n}\right)$.

Next observe that there is an involution $\iota: \widehat{H}_{n}^{a}(q) \rightarrow \widehat{H_{n}^{a}}(q)$ which takes $T_{i}$ to $-T_{i}^{-1}$ $(i=1, \ldots, n-1)$ and $X_{j}$ to $X_{j}^{-1}(j=1, \ldots, n)$; this follows by noting that the images of the $T_{i}$ and $X_{j}$ under $\iota$ satisfy relations in Proposition (3.3)(iv). It follows that for any $\widehat{H_{n}^{a}}(q)$-module $M$, we may define its inflation $\iota^{*} M$ via $\iota$ in the usual way.

(9.6) THEOREM. - Let $R$ be any field, and suppose the pair $(q, z)$ of invertible elements of $R$ is generic (see (8.1)). Assume there is an element $\beta \in R^{\times}$such that $\beta^{2}=-q^{n-2}$. Let $\psi_{\alpha}: \widehat{H_{n}^{a}}(q) \rightarrow T_{n}^{a}(q)\left(\alpha \in R^{\times}\right)$be the surjection of (5.12) (see also (5.13) and (4.3)(i)) and let $W_{t, z}(n)$ be a cell module for the diagram algebra $T_{n}^{a}(q)$, where $t \in \mathbb{Z}, 0 \leqslant t \leqslant n, n-t \in 2 \mathbb{Z}$ and $z \in R^{\times}$. Let $M$ be the $\widehat{H_{n}^{a}}(q)$-module $\iota^{*} \psi_{\alpha}^{*} W_{t, z}(n)$. Then $M$ is isomorphic to the induced module $K_{s_{\lambda}, N_{\lambda}}$ (see (9.4)) where $\lambda$ is the partition $\left(\frac{n+t}{2}, \frac{n-t}{2}\right)$ and $s_{\lambda}$ is the diagonal matrix $\operatorname{diag}\left(s_{1}, \ldots, s_{n}\right)$, where the $s_{i}$ are given by

$$
\left(s_{1}, \ldots, s_{n}\right)=\left(a_{1}, a_{1} q^{-2}, \ldots, a_{1} q^{-(n+t-2)}, a_{2}, a_{2} q^{-2}, \ldots, a_{2} q^{-(n-t-2)}\right),
$$

where $a_{1}=(-1)^{t+1} \alpha z q^{\frac{1}{2}(n+t-2)}$ and $a_{2}=(-1)^{t+1} \alpha z^{-1} q^{\frac{1}{2}(n-t-2)}$.

Proof. - Note first that from diagram (5.12), we see that if $\alpha, \beta$ and $\mu$ are related by $\beta^{2}=-q^{n-2}$ and $\mu \alpha^{-1}=\beta$, then (cf. (7.1))

$$
\psi_{\alpha}^{*} W_{t, z}(n) \cong \psi_{\alpha}^{*} g_{\beta}^{*} W_{t}(n) \cong \xi_{Q, \mu}^{*} W_{t}(n)
$$

where $W_{t}(n)$ is the cell module for $T L B_{n}(q, Q)$ and $z=z_{t}$ (see Theorem (6.1)). But given $t, z$ and $\beta$, (6.12) shows that $W_{t, z}(n) \cong g_{\beta}^{*} W_{t}(n)=g_{\beta, Q}^{*} W_{t}(n)$, where 


$$
Q=Q(t, z)=(-1)^{t} \beta z^{-1} q^{-\frac{1}{2}(n+t-2)} .
$$

Moreover $Q(t, z)^{2}=-z^{-2} q^{-t}$, whence if $(q, z)$ is generic, so is $(q, Q)$.

It follows from (8.5) and (8.7) that there is a vector $E \in M$ such that $X_{i} E=\delta_{i} E$ for $i=1,2, \ldots, n$, where $\left(\delta_{1}, \ldots, \delta_{n}\right)$ is the sequence in (8.5) and $T_{j} E=-q^{-1} E$ for $j=1,2, \ldots$, $n-1, j \neq \frac{n+t}{2}$. Hence $\iota\left(X_{i}\right) E=\delta_{i}^{-1} E$ and $\iota\left(T_{j}\right) E=q E$ for each relevant $i, j$. Since $M$ is irreducible because we are in the generic case, $E$ generates $M$ as $\widehat{H_{n}^{a}}(q)$-module. It follows from Lemma (9.3) and from the description of the character $\chi$ in (9.4) that $M$ is isomorphic to the induced module $K_{s, N_{\lambda}}$, where $\lambda=\left(\frac{n+t}{2}, \frac{n-t}{2}\right)$ and $s$ is the diagonal matrix with diagonal entries

$$
\begin{gathered}
\left(\delta_{1}, \ldots, \delta_{n}\right)=\left(-\mu Q^{-1},-\mu Q^{-1} q^{-2}, \ldots,-\mu Q^{-1} q^{-(n+t-2)}, \mu Q,\right. \\
\left.\mu Q q^{-2}, \ldots, \mu Q q^{-(n-t-2)}\right),
\end{gathered}
$$

where $Q=Q(t, z)=(-1)^{t} \beta z^{-1} q^{-\frac{1}{2}(n+t-2)}$.

Using the relations among $\alpha, \beta, z$ and $Q$, we see that $\mu Q=(-1)^{t+1} \alpha z^{-1} q^{\frac{1}{2}(n-t-2)}$ and $-\mu Q^{-1}=(-1)^{t+1} \alpha z q^{\frac{1}{2}(n+t-2)}$.

(9.7) Notation. - We fix here some notation for the rest of this work. First, if $t \in \mathbb{Z}$, $0 \leqslant t \leqslant n, n-t \in 2 \mathbb{Z}$, we shall consistently write

$$
k=\frac{n-t}{2} .
$$

Corresponding to each such $t$ (or $k$ ) we have the 2-step partition $\lambda_{t}=(n-k, k)$. Note that $n-k \geqslant k$.

The diagonal matrix in the statement of (9.6) will be denoted $s_{k}\left(a_{1}, a_{2}\right)$ or just $s\left(a_{1}, a_{2}\right)$, i.e.

$$
\begin{aligned}
s\left(a_{1}, a_{2}\right) & =s_{k}\left(a_{1}, a_{2}\right)=\operatorname{diag}\left(s_{1}, \ldots, s_{n}\right) \\
& =\operatorname{diag}\left(a_{1}, a_{1} q^{-2}, \ldots, a_{1} q^{-(n+t-2)}, a_{2}, a_{2} q^{-2}, \ldots, a_{2} q^{-(n-t-2)}\right) .
\end{aligned}
$$

Recall the definition of the induced module $K_{\lambda, \chi}$ where $\lambda$ is a partition of $n$ and $\chi$ is a character of $U(n)$ which satisfies condition (9.1). A special case of this definition is when $\lambda=\lambda_{t}$ as above, and $\chi$ corresponds to the semisimple element $s_{k}\left(a_{1}, a_{2}\right)$ of (9.7.2), i.e. $\chi\left(X_{j}\right)=s_{j}^{-1}$. We denote the corresponding induced module of the statement of (9.6) in this case by $K_{s, N}=K_{s\left(a_{1}, a_{2}\right), N_{k}}$, where $N_{k}=N_{\lambda_{t}}$.

We next wish to identify the Grothendieck class of the module $M$ of (9.6) (i.e. its set of composition factors) in general, i.e. when $(q, z)$ is not necessarily a generic pair. We shall prove

(9.8) THEOREM. - Let $F$ be a field, and let $(q, z)$ be a pair of invertible elements of $F$. Assume that there are elements $\sqrt{q}, \sqrt{-1} \in F$ such that $\sqrt{q}^{2}=q$ and $\sqrt{-1}^{2}=-1$. Let $M$ be the $\widehat{H_{n}^{a}}(q)$-module $\iota^{*} \psi_{\alpha}^{*} W_{t, z}(n)$ as in (9.6), where $\widehat{H_{n}^{a}}(q)$ is now an F-algebra, and $\alpha \in F^{\times}$. Then in the Grothendieck group of finite dimensional $\widehat{H_{n}^{a}}(q)$-modules, $M$ is equivalent to the induced module $K_{s_{\lambda}, N_{\lambda}}$ (see (9.4)) where $\lambda, s_{\lambda}$ and $N_{\lambda}$ are as in (9.6).

Proof. - Let $R_{0}=F[\mathbf{q}]$ be the ring of polynomials over $F$ in the indeterminate $\mathbf{q}$. Let $R$ be the completion of $R_{0}$ at the prime ideal $\mathfrak{P}=(\mathbf{q}-\sqrt{q})$, and let $L$ be the quotient field of $R$. Thus if we write $y=\mathbf{q}-\sqrt{q} \in R_{0}, R$ is identified with the ring of power series $F[[y]]$, and $L=F[[y]]_{y}=F[[y]]\left[y^{-1}\right]$. The ring $R$ is then a complete rank one discrete valuation ring with residue field $F$. Under the residue class map $R \rightarrow F, \mathbf{q}^{2} \mapsto q$. 
Consider the affine Hecke algebra $\widehat{H_{n}^{a}}\left(\mathbf{q}^{2}\right)$ over the ring $R$, corresponding to the invertible element $\mathbf{q}^{2} \in R$. The pair $\left(\mathbf{q}^{2}, z\right)$ is generic in $R$, and we may therefore interpret $W_{t, z}(n)$ as a module for the $R$-algebra $T_{n}^{a}\left(\mathbf{q}^{2}\right)$. Write $\widehat{W_{t, z}(n)}$ for this $R$-module. Let $\mathfrak{b} \in R$ satisfy $\mathfrak{b}^{2}=-\mathbf{q}^{2(n-2)}$. Such an element obviously exists, and under the residue class map $\mathfrak{b} \mapsto \beta \in F$ such that $\beta^{2}=-q^{-(n-2)}$.

Write $\mathbf{Q}=(-1)^{t} \mathfrak{b} z^{-1} \mathbf{q}^{-(n+t-2)}$, and denote $R$-modules by means of a tilde. Then by Theorem (6.1), with the obvious extensions of our standard notation,

$$
\psi_{\alpha}^{*} \widetilde{W_{t, z}(n)} \cong \psi_{\alpha}^{*} g_{\mathfrak{b}}^{*} \widetilde{W_{t}(n)} \cong \xi_{\mathbf{Q}, \mathfrak{m}}^{*} \widetilde{W_{t}(n)},
$$

where $\mathfrak{m}=\alpha \mathfrak{b}$.

Since the pair $\left(\mathbf{q}^{2}, z\right)$ is generic in $R$, we may invoke Theorem (9.6) to deduce that

$$
L \otimes_{R} \widetilde{M}=L \otimes_{R} \iota^{*} \psi_{\alpha}^{*} \widetilde{W_{t, z}(n)} \cong L \otimes_{R} \widetilde{K_{s_{\lambda}, N_{\lambda}}} .
$$

The isomorphism (9.8.2) is an isomorphism of $L \otimes_{R} \widehat{H_{n}^{a}}\left(\mathbf{q}^{2}\right)$-modules. We shall show how to interpret it in terms of $L \otimes_{R} T L B_{n}\left(\mathbf{q}^{2}, \mathbf{Q}\right)$-modules, where $T L B_{n}\left(\mathbf{q}^{2}, \mathbf{Q}\right)$ is the $R$-algebra defined in (5.3), corresponding to the parameters $\mathbf{q}^{2}, \mathbf{Q}$.

The left side of (9.8.2) is by (9.8.1) the pullback to $\widehat{H_{n}^{a}}\left(\mathbf{q}^{2}\right)$ of a $T L B_{n}\left(\mathbf{q}^{2}, \mathbf{Q}\right)$-module. Hence the kernel of $\xi_{\mathbf{Q}, \mathfrak{m}}$ acts trivially on the right side of (9.8.2) and it follows easily that the kernel of $\xi_{\mathbf{Q}, \mathfrak{m}}$ acts trivially on the $R$-module $\widetilde{K_{s_{\lambda}, N_{\lambda}}}$, whence the latter may be thought of as a $T L B_{n}\left(\mathbf{q}^{2}, \mathbf{Q}\right)$-module. Thus the isomorphism (9.8.2) may be interpreted as an isomorphism of $L \otimes_{R} T L B_{n}\left(\mathbf{q}^{2}, \mathbf{Q}\right)$ modules. Moreover we may think of the space of either side as an $L$ vector space, in which there are two $R$-lattices invariant under $T L B_{n}\left(\mathbf{q}^{2}, \mathbf{Q}\right)$, which correspond respectively to the representations $\widetilde{M}$ and $\widetilde{K_{s_{\lambda}, N_{\lambda}}}$ of the $R$-algebra $T L B_{n}\left(\mathbf{q}^{2}, \mathbf{Q}\right)$.

Now since the pair $\left(\mathbf{q}^{2}, z\right)$ is generic in $R$, and therefore generic in $L$, the $L \otimes_{R} T L B_{n}\left(\mathbf{q}^{2}, \mathbf{Q}\right)$ module $L \otimes_{R} \widehat{W_{t}(n)}$ is absolutely irreducible, whence $L$ is a splitting field for

$$
L \otimes_{R} \widetilde{M} \cong L \otimes_{R} \widetilde{K_{s_{\lambda}, N_{\lambda}}} .
$$

The reductions mod $\mathfrak{P}$ of $\widetilde{M}$ and $\widetilde{K_{s_{\lambda}, N_{\lambda}}}$ are respectively isomorphic to the $T L B_{n}(q, Q)$ modules $M$ and $K_{s_{\lambda}, N_{\lambda}}$, and since $F$ is a splitting field for $T L B_{n}(q, Q)$ (cf. [14, §5]), the triple $(L, R, F)$ is a splitting system for $T L B_{n}\left(\mathbf{q}^{2}, \mathbf{Q}\right)$ in the sense of [3, p. 17]. Moreover since $\left(\mathbf{q}^{2}, z\right)$ is generic in $L, L \otimes_{R} T L B_{n}\left(\mathbf{q}^{2}, \mathbf{Q}\right)$ is semisimple. It follows from [op. cit., Proposition 1.9.6, p. 19], that the $T L B_{n}(q, Q)$ modules (and hence the $\widehat{H_{n}^{a}}(q)$-modules) $M$ and $K_{s_{\lambda}, N_{\lambda}}$ have the same composition factors, counting multiplicity, which is the required statement.

The above result enables us to determine the composition factors of the standard modules $M_{s, N}$ for any pair $s, N$, where $N$ has at most two Jordan blocks (we refer to such $N$ as "twostep nilpotent") and when $q$ is arbitrary. It has the following consequence.

(9.9) COROLLARY. - Let $\widehat{H_{n}^{a}}(q)$ be the affine Hecke algebra over the complex numbers $\mathbb{C}$ and let $\Gamma\left(\widehat{H_{n}^{a}}(q)\right)$ be the Grothendieck group of finite dimensional $\widehat{H_{n}^{a}}(q)$-modules. Let $\lambda=(n-k, k)$ be a 2-step partition of $n(2 k \leqslant n)$, let $t=n-2 k$ and let $s$ be the diagonal matrix of (9.7.2). Write $M_{s, N}$ for the Kazhdan-Lusztig standard module of $\widehat{H_{n}^{a}}(q)$ corresponding to the pair $s=s_{k}(a, b), N=N_{k}(c f .(9.7))$. Then

$$
\left[M_{s, N}\right]=\left[\theta_{\alpha}^{*} W_{t, z}(n)\right]
$$


where $[V]$ denotes the class in $\Gamma\left(\widehat{H_{n}^{a}}(q)\right)$ of an $\widehat{H_{n}^{a}}(q)$-module $V, W_{t, z}(n)$ is a cell module for the diagram algebra $T_{n}^{a}(q), \theta_{\alpha}: \widehat{H_{n}^{a}}(q) \rightarrow T_{n}^{a}(q)$ is the surjection $\psi_{\alpha}$ of (5.12) composed with the involution $\iota$ of (9.6), and $\alpha, z, t$ satisfy the equations

$$
\begin{aligned}
& k=\frac{n-t}{2}, \\
& a=(-1)^{n+1} \alpha z q^{\frac{1}{2}(n+t-2)}, \\
& b=(-1)^{n+1} \alpha z^{-1} q^{\frac{1}{2}(n-t-2)} .
\end{aligned}
$$

Proof. - This follows easily from (9.5) and (9.8). The former asserts that $\left[M_{s, N}\right]=\left[K_{s, N}\right]$, while the latter asserts that $\left[K_{s, N}\right]=\left[\theta_{\alpha}^{*} W_{t, z}(n)\right]$. The formulae for $a$ and $b$ are in the statement of (9.6), taking account of the fact that $t$ and $n$ have the same parity.

We shall use (9.9) to study the composition factors of the standard modules $M_{s, N}$ through our knowledge of those of the cell modules $W_{t, z}(n)$. First we need to understand the correspondence between cell modules and standard modules a little better. Assume henceforth that the ground ring $R$ is $\mathbb{C}$.

(9.10) Corollary. - Given the pair $\left(s(a, b), N_{k}\right)$ (see (9.7)) with $2 k \leqslant n$, there exist $(t, \alpha, z)$ such that $\left[M_{s(a, b), N_{k}}\right]=\left[\theta_{\alpha}^{*} W_{t, z}(n)\right]$. The parameters $(t, \alpha, z)$ are given by

$$
\begin{aligned}
t & =n-2 k, \\
\alpha^{2} & =a b q^{-(n-2)}, \\
z & =(-1)^{n+1} \alpha^{-1} a q^{-\frac{1}{2}(n+t-2)}=(-1)^{n+1} \alpha b^{-1} q^{\frac{1}{2}(n-t-2),}
\end{aligned}
$$

where, if $t=n($ or $k=0)$, the second equation $\left(\right.$ for $\left.\alpha^{2}\right)$ is omitted.

(9.11) LEMMA. - We have $\psi_{\alpha}^{*} W_{t, z}(n) \cong \psi_{-\alpha}^{*} W_{t,-z}(n)$.

Proof. - For any element $\beta \in \mathbb{C}$ such that $\beta^{2}=-q^{n-2}$, and $\alpha, \mu \in \mathbb{C}$ such that $\mu=\alpha \beta$, we have a commutative diagram (5.12) for any $Q \in \mathbb{C}$, and by (6.12), $W_{t, z}(n)=g_{\beta, Q}^{*} W_{t}(n)$, where $Q=Q(t, z)=(-1)^{t} \beta z^{-1} q^{-\frac{1}{2}(n+t-2)}$. Hence $\psi_{\alpha}^{*} W_{t, z}(n) \cong \psi_{\alpha}^{*} g_{\beta, Q}^{*} W_{t}(n) \cong \xi_{Q, \mu}^{*} W_{t}(n)$. If we replace $\beta$ by $-\beta$ and $\alpha$ by $-\alpha, \mu$ is unchanged. Thus replacing $z$ by $-z$ leaves $Q$ unchanged, and it follows that we also have $\xi_{Q, \mu}^{*} W_{t}(n) \cong \psi_{-\alpha}^{*} W_{t,-z}(n)$, as stated.

(9.12) COROLlary. - Let $\mathcal{P}$ be the set equivalence classes of pairs $(s, N) \in \widehat{\mathcal{P}}$ where $N \in \mathfrak{G}$ is two-step nilpotent, i.e. $N \sim N_{k}$ for some $k$ with $0 \leqslant 2 k \leqslant n$. Let $\widetilde{\Omega}$ be the set of triples $(t, \alpha, z)\left(t \in \mathbb{Z}, 0 \leqslant t \leqslant n, n-t \in 2 \mathbb{Z} ; \alpha, z \in \mathbb{C}^{\times}\right)$and let $\Omega$ be the set of equivalence classes of triples in $\widetilde{\Omega}$ under the equivalence generated by the relations $(t, \alpha, z) \sim(t,-\alpha,-z)$, $(n, \alpha, z) \sim\left(n, y^{-1} z \alpha, y\right)$ and $(0, \alpha, z) \sim\left(0, \alpha, z^{-1}\right)$. Then (with the obvious abuse of notation) we have well defined $\widehat{H_{n}^{a}}(q)$-modules $M_{s, N},(s, N) \in \mathcal{P}$ and $\theta_{\alpha}^{*} W_{t, z}(n)($ see $(9.9)),(t, \alpha, z) \in \Omega$, and there is a bijection $f: \mathcal{P} \rightarrow \Omega$ such that if $(s, N) \in \mathcal{P}$ corresponds to $(t, \alpha, z) \in \Omega$, $\left[M_{s, N}\right]=\left[\theta_{\alpha}^{*} W_{t, z}(n)\right]$.

Proof. - The modules $M_{s, N}$ are the standard modules of Kazhdan-Lusztig. We need to verify that the isomorphism class of $\theta_{\alpha}^{*} W_{t, z}(n)$ depends only on the equivalence class of $(t, \alpha, z)$. But Lemma (9.11) proves this for one type of equivalence, while if $t=0$, we observe that $W_{0, z}(n) \cong W_{0, z^{-1}}(n)$, and if $t=n$, in the relations (9.9.1) only $a$ occurs, since $N_{k}=N_{0}$ 
has only one Jordan block, and clearly $a$ depends only on $\alpha z$, so that for any $y, z, \alpha \in \mathbb{C}^{\times}$, $\theta_{\alpha}^{*} W_{n, z}(n) \cong \theta_{y^{-1} z \alpha}^{*} W_{n, y}(n)$, which completes the verification.

Next, let us define the required bijection $f: \Omega \rightarrow \mathcal{P}$. Given $(t, \alpha, z) \in \Omega$, define $f(t, \alpha, z)$ to be the class of $\left(s(a, b), N_{k}\right)$, where $a, b, k$ are given by Eqs. (9.9.1). To check that this is well defined, note that if $(t, \alpha, z)$ is replaced by $(t,-\alpha,-z)$, Eqs. (9.9.1) are unchanged, and therefore have the same solution. If $(0, \alpha, z)$ is replaced by $\left(0, \alpha, z^{-1}\right)$, we obtain the solution $\left(s(b, a), N_{\frac{n}{2}}\right)$ of (9.9.1). But the pairs $\left(s(b, a), N_{\frac{n}{2}}\right)$ and $\left(s(a, b), N_{\frac{n}{2}}\right)$ are conjugate under $G L_{n}(\mathbb{C})$. Finally, the triples $(n, \alpha, z)$ and $\left(n, y^{-1} z \alpha, y\right)$ give the same values of $k(=0)$ and $a$, which proves that $f$ is well defined.

Finally, define $f^{\prime}: \mathcal{P} \rightarrow \Omega$ by taking $f^{\prime}\left(s(a, b), N_{k}\right)$ to be the equivalence class of any triple $(t, \alpha, z)$ where the parameters satisfy Eqs. (9.10.1). If $t \neq n$ there are just two such triples, which are obviously equivalent. If $t=n$, all the resulting triples are easily seen to be equivalent, since they have a common value of $\alpha z$. To check that $f^{\prime}$ is well defined, we note that the pairs $\left(s(a, b), N_{k}\right)$ and $\left(s\left(a^{\prime}, b^{\prime}\right), N_{k^{\prime}}\right)$ are conjugate under $G L_{n}(\mathbb{C})$ if and only if either they are equal, or $k=k^{\prime}=n / 2$ and $\left(a^{\prime}, b^{\prime}\right)=(b, a)$. In the latter case, solving (9.10.1) shows that the corresponding triples both have $t=0$ and $z$ in one is replaced by $z^{-1}$ in the other, whence the triples are equivalent, which shows that $f^{\prime}$ is well defined. Since $f, f^{\prime}$ are clearly inverses, both are bijections, and the result now follows from Theorem (9.8).

(9.13) Remark. - There is a close analogy between the set $\mathcal{P}$ described here and the "multisegments" of Zelevinsky [31, §4]. We shall elaborate on this in Section 11 below.

\section{Irreducible modules and decomposition numbers}

We begin by reviewing the main points concerning the representation theory of the algebra $T_{n}^{a}(q)$. In this section, $R$ is a field.

(10.1) Definition. - Let $\Lambda^{a}(n)^{+}$be the set

$$
\Lambda^{a}(n)^{+}=\left\{(t, z) \mid t \in \mathbb{Z}_{\geqslant 0}, 0 \leqslant t \leqslant n, n-t \in 2 \mathbb{Z} ; z \in R^{\times}\right\} .
$$

Define $\Lambda^{a}(n)$ by

$$
\Lambda^{a}(n)= \begin{cases}\Lambda^{a}(n)^{+} & \text {if } q^{2} \neq-1 \\ \Lambda^{a}(n)^{+} \backslash\{(0, \pm q)\} & \text { if } q^{2}=-1\end{cases}
$$

Define the equivalence relation $\approx$ on $\Lambda^{a}(n)^{+}$as that which identifies $(0, z)$ and $\left(0, z^{-1}\right)$ for all $z \in R^{\times}$, and write

$$
\begin{gathered}
\Lambda^{a}(n)^{0}=\Lambda^{a}(n) / \approx, \\
\Lambda^{a}(n)^{0+}=\Lambda^{a}(n)^{+} / \approx .
\end{gathered}
$$

Recall (e.g. from (6.6) above) that if $X_{t}$ is the $T_{n}^{a}(q)$-module with basis all monic affine diagrams : $t \rightarrow n$, the cell module $W_{t, z}(n)$ for $T_{n}^{a}(q)$ is defined as the quotient of $X_{t}$ by the ideal $I_{\chi}:=\left\{\gamma \tau_{t}-\chi \gamma\right\}$ for $\gamma \in X_{t}$, where $\chi=\chi_{z}=z$ if $t \neq 0$ and $\chi=\chi_{z}=z+z^{-1}$ if $t=0$. There is a bilinear map $\phi_{t, z}: W_{t, z}(n) \times W_{t, z^{-1}}(n) \rightarrow R$ which [14, (2.7)] is invariant in the sense that $\phi_{t, z}(w \mu, \nu)=\phi_{t, z}\left(\mu, w^{*} \nu\right),\left(\mu, \nu \in W_{t, z}(n), w \in T_{n}^{a}(q)\right)$ where $d^{*}$ denotes the reflection of a diagram $d \in \mathcal{T}$ in a horizontal line. Note that $w \mapsto w^{*}$ is an anti-automorphism of $T_{n}^{a}(q)$, which preserves $T L_{n}^{a}(q)$. If $R$ is a field, the irreducible $T_{n}^{a}(q)$-modules have the following description. 
(10.2) THEOREM [14, (2.8)]. - Let $R$ be an algebraically closed field and maintain the above notation. For $\left(t_{1}, z_{1}\right),\left(t_{2}, z_{2}\right) \in \Lambda^{a}(n)^{+}, W_{t_{1}, z_{1}}(n) \cong W_{t_{2}, z_{2}}(n)$ if and only if $\left(t_{1}, z_{1}\right) \approx\left(t_{2}, z_{2}\right)$. For $(t, z) \in \Lambda^{a}(n)^{+}, L_{t, z}(n):=W_{t, z}(n) / \operatorname{rad} \phi_{t, z}$ is either an irreducible $T_{n}^{a}(q)$ module or zero, and $L_{t, z}(n) \neq 0$ if and only if $(t, z) \in \Lambda^{a}(n)$ (see (10.1.1)). All irreducible $T_{n}^{a}(q)$ modules are realised thus, and if $\left(t_{1}, z_{1}\right) \not\left(t_{2}, z_{2}\right)$, then $L_{t_{1}, z_{1}}(n) \neq L_{t_{2}, z_{2}}(n)$.

It follows that the distinct irreducible $T_{n}^{a}(q)$-modules are parametrised by $\Lambda^{a}(n)^{0}$, while the distinct cell modules are parametrised by $\Lambda^{a}(n)^{0+}$. Where there is little danger of confusion, we abuse notation by denoting the elements of $\Lambda^{a}(n)^{0+}$ as pairs $(t, z)$, rather than equivalence classes of pairs. Thus we speak of $W_{t, z}(n)$ and $L_{t, z}(n)$ for $(t, z) \in \Lambda^{a}(n)^{0+}$. It follows from (e.g.) (9.12) that to understand the composition factors of the $\widehat{H_{n}^{a}}(q)$-module $M_{s, N}((s, N) \in \mathcal{P})$, it suffices to understand those of the $T_{n}^{a}(q)$-module $W_{t, z}(n)$ for appropriate $(t, z) \in \Lambda^{a}(n)^{+}$. We therefore now turn to a description of these, which is available from [14].

Let $\preceq$ be the partial order on $\Lambda^{a}(n)^{+}$which is generated by the preorder $\stackrel{\circ}{\prec}$ which stipulates that $(t, z) \stackrel{\circ}{\prec}(s, y)$ if

$$
\begin{aligned}
& 0 \leqslant t \leqslant s \leqslant n, \quad s=t+2 \ell(\ell \in \mathbb{Z}, \ell>0) \quad \text { and } \\
& z^{2}=q^{\varepsilon(s, z) s} \text { and } y=z q^{-\varepsilon(s, z) \ell} \text { for } \varepsilon(s, z)= \pm 1 .
\end{aligned}
$$

Note that $(10.3 \mathrm{a}, \mathrm{b})$ implies that

$$
y^{2}=q^{\varepsilon(s, z) t} \quad \text { and } \quad z^{t}=y^{s}
$$

and

$$
(t, z) \preceq\left(t^{\prime}, z^{\prime}\right) \Rightarrow z^{t}=\left(z^{\prime}\right)^{t^{\prime}} .
$$

It suffices to verify (10.4b) when $(t, z) \stackrel{\circ}{\prec}\left(t^{\prime}, z^{\prime}\right)$, in which case it follows easily from (10.3a,b).

It is easily verified that $[15,4.1]$ the partial order $\preceq$ on $\Lambda^{a}(n)^{+}$induces a partial order, also denoted $\preceq$, on the set $\Lambda^{a}(n)^{0}=\Lambda^{a}(n) / \approx$.

The following result is proved in [14, Theorem 5.1].

(10.5) TheOREM. - Let $R$ be a field of characteristic 0 or $p>0$, where pe $>n$ and $e$ is the multiplicative order of $q^{2}$. Then, in the Grothendieck group $\Gamma\left(T_{n}^{a}(q)\right)$, we have for any $(t, z) \in \Lambda^{a}(n)^{+}$,

$$
W_{t, z}(n)=\sum_{\substack{(s, y) \in \Lambda^{a}(n)^{0} \\(t, z) \preceq(s, y)}} L_{s, y}(n) .
$$

Thus the matrix expressing the cell modules in terms of the irreducibles in $\Gamma\left(T_{n}^{a}(q)\right)$ is upper unitriangular, and has entries 0 or 1 . Now if $(t, z)$ is confined to $\Lambda^{a}(n)$, the relation (10.5.1) can clearly be inverted.

We also have (cf. [15, Theorem (4.5)])

(10.6) THEOREM. - In the notation above, if $(t, z) \in \Lambda^{a}(n)$,

$$
L_{t, z}(n)=\sum_{\substack{(s, y) \in \Lambda^{a}(n)^{0} \\(t, z) \preceq(s, y)}} n_{t, z}^{s, y} W_{s, y}(n)
$$

where $n_{t, z}^{s, y}=0$ or \pm 1 . 
We next explore the implications of these results for the standard modules $M_{s, N}$.

(10.7) Definition. - For any triple $(t, \alpha, z)$ where $(t, z) \in \Lambda^{a}(n)^{+}$and $\alpha \in \mathbb{C}^{\times}$, define the modules

$$
\begin{aligned}
W_{t, z}(\alpha) & :=\theta_{\alpha}^{*} W_{t, z}(n), \\
L_{t, z}(\alpha) & :=\theta_{\alpha}^{*} L_{t, z}(n) .
\end{aligned}
$$

(10.8) Lemma. - All composition factors of the cell module $W_{t, z}(\alpha)$ are of the form $\theta_{\alpha}^{*} L_{t^{\prime}, z^{\prime}}(n)$ for some triple $\left(t^{\prime}, \alpha, z^{\prime}\right)$.

Proof. - It is clear that the pullback via $\theta_{\alpha}$ of a composition series for $W_{t, z}(n)$ is a composition series for the $\widehat{H_{n}^{a}}(q)$-module $W_{t, z}(\alpha)$. The result therefore follows from Theorem (10.2).

We need to identify which among the $L_{t, z}(\alpha)$ are distinct. For this, we shall regard the modules we are considering as $\widehat{T L_{n}^{a}}(q)$-modules, via the commutative diagram

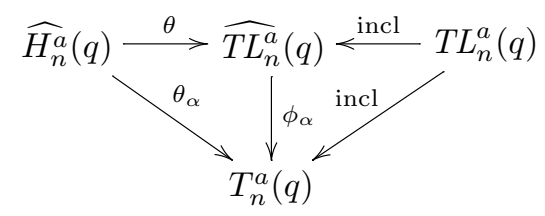

where $\theta=\eta_{3} \circ \iota, \eta_{3}$ is as in (5.6) or (5.9), $\iota$ is the involution of $\widehat{H_{n}^{a}}(q)$ defined immediately preceding the statement of Theorem (9.6) above and $\theta_{\alpha}$ is defined in (9.9).

(10.10) Proposition. - The $\widehat{H_{n}^{a}}(q)$-modules $L_{t, z}(\alpha)=\theta_{\alpha}^{*} L_{t, z}(n)$ for $(t, z) \in \Lambda^{a}(n)$, $\alpha \in \mathbb{C}^{\times}$, are precisely those irreducible $\widehat{H_{n}^{a}}(q)$-modules which factor through $\widehat{T L_{n}^{a}}(q)$.

Proof. - The statement is equivalent to the assertion that any irreducible $\widehat{T L_{n}^{a}}(q)$-module is of the form $\phi_{\alpha}^{*} L_{t, z}(n)$. To prove this, let $M$ be any irreducible finite dimensional $\widehat{T L_{n}^{a}}(q)$ module. Recall (see (4.2) above) that $T L_{n}^{a}(q)=\left\langle C_{1}, \ldots, C_{n}\right\rangle$ is a subalgebra of $\widehat{T L_{n}^{a}}(q)$ and $\widehat{T L_{n}^{a}}(q) \cong \mathbb{C}\langle V\rangle \otimes_{\mathbb{C}} T L_{n}^{a}(q)$, where $V$ is the automorphism of $T L_{n}^{a}(q)$ which permutes the $C_{i}$ cyclically.

Let $M_{1}$ be an irreducible $T L_{n}^{a}(q)$-submodule of $M$. Then by [15, (2.3)], $M_{1} \cong L_{t, z}(n)$ or $M_{1} \cong L_{0, \sqrt{-1}}^{ \pm}(n)$. If $M_{1} \cong L_{t, z}(n)$, then by $[15,(2.5)]$ the twist $M_{1}^{V}$ of $M_{1}$ by the automorphism $V$ of $T L_{n}^{a}(q)$ is isomorphic to $L_{t, z}(n)$. It follows from [15, Theorem (2.6)] that as a vector space, $M=L_{t, z}(n)$. Moreover by Schur's Lemma, $V$ acts as a scalar multiple of $\tau_{n}$, i.e. as $\alpha \tau_{n}$ for some $\alpha \in \mathbb{C}^{\times}$, so that $M \cong \phi_{\alpha}^{*} L_{t, z}(n)$. In the other case, [op. cit. Theorem (2.6)] shows that as vector space $M \cong L_{0, \sqrt{-1}}^{+}(n) \oplus L_{0, \sqrt{-1}}^{-}(n)$, and following the argument in loc. cit. further shows that $M \cong \phi_{\alpha}^{*} L_{0, \sqrt{-1}}(n)$ for some $\alpha \in \mathbb{C}^{\times}$.

(10.11) Definition. - Denote by $\widetilde{\Omega}_{0}$ the set of triples $(t, \alpha, z)$ in $\widetilde{\Omega}$ (see the definition in (9.12)) such that $(t, z) \in \Lambda^{a}(n)$. Then $\widetilde{\Omega}_{0}$ is a union of $\sim$-classes of $\widetilde{\Omega}$ and we write $\Omega_{0}=\widetilde{\Omega}_{0} / \sim$ for the corresponding set of equivalence classes.

The triples $(t, \alpha, z)$ in $\widetilde{\Omega}$ are by Theorem (10.2) precisely those such that the corresponding module $L_{t, z}(\alpha)$ is non-zero.

We are now in a position to determine the coincidences among the irreducible modules $L_{t, z}(\alpha)$ for different values of the parameters. 
(10.12) TheOREM. - Let $(t, \alpha, z)$ and $\left(t^{\prime}, \alpha^{\prime}, z^{\prime}\right)$ be two triples in $\widetilde{\Omega}_{0}$ (see Definition (10.11)). The irreducible $\widehat{T L_{n}^{a}}(q)$-modules $L_{t, z}(\alpha)$ and $L_{t^{\prime}, z^{\prime}}\left(\alpha^{\prime}\right)$ are isomorphic if and only if the triples $(t, \alpha, z)$ and $\left(t^{\prime}, \alpha^{\prime}, z^{\prime}\right)$ are equivalent, i.e. represent the same element of $\Omega($ see (9.12)).

Proof. - We first show that if $(t, \alpha, z) \sim\left(t^{\prime}, \alpha^{\prime}, z^{\prime}\right)$ then $L_{t, z}(\alpha) \cong L_{t^{\prime}, z^{\prime}}\left(\alpha^{\prime}\right)$. For this, observe that $W_{t, z}(\alpha) \cong W_{t,-z}(-\alpha)$ for all triples $(t, \alpha, z) \in \widetilde{\Omega}$, that $W_{0, z}(\alpha) \cong W_{0, z^{-1}}(\alpha)$ for all $\alpha, z$, and that $L_{n, z}(\alpha)=W_{n, z}(\alpha) \cong W_{n, y}\left(y^{-1} z \alpha\right)=L_{n, y}\left(y^{-1} z \alpha\right)$. It follows by taking top quotients that if $(t, \alpha, z) \sim\left(t^{\prime}, \alpha^{\prime}, z^{\prime}\right)$ then $L_{t, z}(\alpha) \cong L_{t^{\prime}, z^{\prime}}\left(\alpha^{\prime}\right)$.

To prove the converse, we need to show that for any triple $(t, \alpha, z)$ in $\widetilde{\Omega}_{0}$, the equivalence class of $(t, \alpha, z)$ is determined by the isomorphism class of $\phi_{\alpha}^{*} L_{t, z}(n)$ as $\widehat{T L_{n}^{a}}(q)$-module. Recall (cf. diagram (10.9)) that $T L_{n}^{a}(q)$ is a subalgebra of $\widehat{T L_{n}^{a}}(q)$ and that the restriction of $L_{t, z}(\alpha)$ to $T L_{n}^{a}(q)$ is $L_{t, z}(n)$. It follows from [14, (1.9)] (or [15], or cf. the proof of (10.10) above) that the equivalence class of the pair $(t, z)$ in $\Lambda^{a}(n)$ under the equivalence relation $\equiv$ is determined by $L_{t, z}(\alpha)$, where $\equiv$ is generated by the relations

$$
\begin{aligned}
(t, z) & \equiv(t,-z) \quad \text { if } t \neq 0, n, \\
(n, z) & \equiv(n, y) \quad \text { for any } y, z \in \mathbb{C}^{\times}, \\
(0, z) & \equiv\left(0, z^{-1}\right) \quad \text { for } z \in \mathbb{C}^{\times} .
\end{aligned}
$$

Further, recall that $V \in \widehat{T L_{n}^{a}}(q)$ acts on $\phi_{\alpha}^{*} L_{t, z}(n)$ as $\alpha \tau_{n} \in T_{n}^{a}(q)$ does.

Suppose for the moment that $t \neq 0, n$. Then by $(10.12 .1),(t, z)=\left(t^{\prime}, z^{\prime}\right)$ or $\left(t^{\prime},-z^{\prime}\right)$. If $(t, z)=\left(t^{\prime}, z^{\prime}\right)$, then by hypothesis $\phi_{\alpha}^{*} L_{t, z}(n) \cong \phi_{\alpha^{\prime}}^{*} L_{t, z}(n)$, whence there is a linear map $\lambda: \mathcal{D} \rightarrow \mathcal{D}$, where $\mathcal{D}$ is the $\mathbb{C}$-vector space with basis the standard affine diagrams $t \rightarrow n$ (see [14, $\S 2])$, which commutes with the $T L_{n}^{a}(q)$ action, and intertwines the $\widehat{T L_{n}^{a}}(q)$ structures $\phi_{\alpha}^{*} L_{t, z}(n)$ and $\phi_{\alpha^{\prime}}^{*} L_{t, z}(n)$. But in this case the restriction $L_{t, z}(n)$ of $\phi_{\alpha}^{*} L_{t, z}(n)$ to $T L_{n}^{a}(q)$ is irreducible, whence $\lambda$ must be multiplication by a scalar, say $a \in \mathbb{C}$. Moreover we have for $E \in L_{t, z}(n)$,

$$
\lambda(V \cdot E)=\lambda\left(\alpha \tau_{n} . E\right)=a \alpha \tau_{n} . E=V \cdot \lambda(E)=\alpha^{\prime} \tau_{n} \cdot a E=a \alpha^{\prime} \tau_{n} . E,
$$

whence $\alpha=\alpha^{\prime}$. If $(t, z)=\left(t^{\prime},-z^{\prime}\right)$, then since the restrictions of $L_{t, z}(\alpha)$ and $L_{t,-z}\left(\alpha^{\prime}\right)$ to $T L_{n}^{a}(q)$ are isomorphic irreducible representations, any linear map $\lambda: \mathcal{D} \rightarrow \mathcal{D}$ (as above) which intertwines the $L_{t, z}(\alpha)$ and $L_{t,-z}\left(\alpha^{\prime}\right)$-actions on $\mathcal{D}$ is of the form $a U$, where $U$ is the map defined in the proof of Theorem (2.3) of [15], and $a \in \mathbb{C}$ (for any standard diagram $E$, $\left.U . E=(-1)^{\operatorname{rank}(E)} E\right)$. Computation of $U(V . E)$ in two different ways as in (10.12.2) then yields that $\alpha^{\prime}=-\alpha$. This completes the proof that $(t, \alpha, z) \sim\left(t^{\prime}, \alpha^{\prime}, z^{\prime}\right)$ if $t \neq 0, n$.

If $t=n, W_{n, z}(n)$ is one-dimensional, and hence irreducible. Moreover $V$ acts on $\phi_{\alpha}^{*} L_{n, z}(n)$ as multiplication by $\alpha z$. Hence if $t=n$, the isomorphism class of $\phi_{\alpha}^{*} L_{t, z}(n)$ is determined by $\alpha z$, whence the result for this case.

Now suppose that $t=0$. If $z^{2} \neq-1$ (i.e. $z+z^{-1} \neq 0$ ), then the restriction to $T L_{n}^{a}(q)$ of $\phi_{\alpha}^{*} L_{t, z}(n)$ is irreducible, and in both cases $z^{\prime}=z$ and $z^{\prime}=z^{-1}$ an argument like (10.12.2) shows that $\alpha=\alpha^{\prime}$, which shows that $(t, \alpha, z) \sim\left(t^{\prime}, \alpha^{\prime}, z^{\prime}\right)$.

Finally, take $z^{2}=-1$ and $q^{2} \neq-1$. In this case the restriction $M$ to $T L_{n}^{a}(q)$ of $\phi_{\alpha}^{*} L_{t, z}(n)$ is (cf. the proof of (10.10)) the direct sum $L_{0, z}^{+}(n) \oplus L_{0, z}^{-}(n)$. The two (irreducible) direct summands are interchanged by $\tau$, and $V$ therefore acts on $\phi_{\alpha}^{*} L_{t, z}(n)$ via the matrix

$$
\left[\begin{array}{cc}
0 & \alpha \tau_{n} \\
\alpha \tau_{n} & 0
\end{array}\right]
$$


But it is shown in $[15,(2.8 .1)]$ that the isomorphism class of the $\widehat{T L_{n}^{a}}(q)$-module $M$ on which $T L_{n}^{a}(q)$ acts according to the given structure and on which $V$ acts via the matrix

$$
\left[\begin{array}{cc}
0 & \alpha_{1} \tau_{n} \\
\alpha_{2} \tau_{n} & 0
\end{array}\right]
$$

is determined by the product $\alpha_{1} \alpha_{2}$. Hence if $z^{2}=-1, \phi_{\alpha}^{*} L_{0, z}(n) \cong \phi_{\alpha^{\prime}}^{*} L_{0, \pm z}(n)$ if and only if $\alpha^{\prime}= \pm \alpha$, which is the required statement for this case. This completes the proof of Theorem (10.12).

(10.13) Corollary. - In the notation of (9.12), let $(s, N)$ be a pair in $\mathcal{P}$ (recall that this means that $N=N_{k}$ is a 2-step nilpotent matrix and $s=s(a, b)$ is a diagonal matrix such that $\left.s N s^{-1}=q^{2} N\right)$. Then there is a well-defined $\widehat{H_{n}^{a}}(q)$-module $L_{s, N}$, which is either 0 or a composition factor of $M_{s, N}$, with the following properties. The non-zero modules $L_{s, N}$ are pairwise non-isomorphic, and the composition factors of all the modules $M_{s^{\prime}, N^{\prime}}\left(\left(s^{\prime}, N^{\prime}\right) \in \mathcal{P}\right)$ are among the $L_{s, N}$. The condition that $L_{s(a, b), N_{k}}$ be zero is that

(10.13.1) $q^{2}=-1, \quad n$ is even, $k=\frac{n}{2}, \quad a=\alpha, \quad b=-\alpha, \quad$ for some $\alpha \in \mathbb{C}^{\times}$.

Proof. - If $(s, N)$ corresponds to the triple $(t, \alpha, z) \in \Omega$, take $L_{s, N}$ to be the top quotient $L_{t, z}(\alpha)$ of $W_{t, z}(\alpha)$. The fact that the non-zero modules $L_{s, N}$ are distinct follows from (10.12). Moreover, by (9.12), $M_{s, N}$ and $W_{t, z}(\alpha)$ have the same composition factors. But the composition factors of $W_{t, z}(\alpha)$ are clearly the inflations via $\theta_{\alpha}$ of those of the $T_{n}^{a}(q)$-module $W_{t, z}(n)$. The result is now clear from (10.5), which shows that the composition factors of $W_{t, z}(n)$ are all top quotients of some $W t^{\prime}, z^{\prime}(n)$. The last assertion concerning the cases when $L_{s, N}=0$ is obtained simply by translating the condition in Theorem (10.2) (viz. $(t, z)=(0, \pm q)$ when $q^{2}=-1$ ) into the language of pairs using (9.9.1).

Our final task is to translate the precise results $(10.3 \mathrm{a}, \mathrm{b})$ et seq. concerning the composition factors of the modules $W_{t, z}(n)$ into the language of pairs $(s, N)$. From the argument in the proof of (10.13), it is clear that in the Grothendieck group $\Gamma\left(\widehat{H_{n}^{a}}(q)\right)$, for any $(t, z) \in \Lambda^{a}(n)^{+}$and $\alpha \in \mathbb{C}^{\times}$,

$$
W_{t, z}(\alpha)=\sum_{\substack{(s, y) \in \Lambda^{a}(n)^{0} \\(t, z) \preceq(s, y)}} L_{s, y}(\alpha) .
$$

In order to describe the composition multiplicities of the standard modules $M_{s, N}$ for $(s, N) \in \mathcal{P}$, we therefore need to interpret the order relation $\prec$ in terms of pairs.

(10.15) Proposition. - Suppose that under the correspondence of (9.12), the triples $\left(t_{1}, \alpha, z_{1}\right),\left(t_{2}, \alpha, z_{2}\right)$ correspond to the pairs $\left(s\left(a_{1}, b_{1}\right), N_{k_{1}}\right),\left(s\left(a_{2}, b_{2}\right), N_{k_{2}}\right)$ respectively. Then $\left(t_{1}, z_{1}\right) \stackrel{\circ}{\prec}\left(t_{2}, z_{2}\right)$ if and only if there exists $\ell>0$ and $\varepsilon= \pm 1$ such that if we write $2 k_{i}=n-t_{i}$ for $i=1,2$, then

$$
\begin{aligned}
k_{2} & =k_{1}-\ell \geqslant 0, \\
a_{1} b_{1}^{-1} & =q^{t_{1}+\varepsilon t_{2}}, \\
\left(a_{2}, b_{2}\right) & = \begin{cases}\left(a_{1}, b_{1}\right) & \text { if } \varepsilon=1, \\
\left(b_{1}, a_{1}\right) & \text { if } \varepsilon=-1 .\end{cases}
\end{aligned}
$$


Proof. - From Eqs. (9.9.1), we see that $z_{1}^{2}=a_{1} b_{1}^{-1}$. Hence from Eqs. (10.3), $t_{2}=t_{1}+2 \ell$ for some $\ell>0$, and $a_{1} b_{1}^{-1} q^{-t_{1}}=q^{\varepsilon t_{2}}$ for some $\varepsilon= \pm 1$. Since $z_{2}=z_{1} q^{-\varepsilon \ell}$, Eqs. (9.10.1) show that $a_{2}=a_{1} q^{\ell(1-\varepsilon)}$ and $b_{2}=b_{1} q^{-\ell(1-\varepsilon)}$. The relations (10.15.1) follow after a little rearrangement. Conversely, if the relations (10.15.1) hold, Eqs. (9.9.1) and (9.10.1) may be used as above to deduce the relations (10.3a) and (10.3b).

The relation $\stackrel{\bigcirc}{\prec}$ defined by Eqs. (10.15.1) generates a partial order $\preceq$ on the set $\mathcal{P}$ of pairs $(s, N)$ described in (9.12). This partial order may be interpreted as ordering the $G$-conjugacy classes of pairs in the obvious way; viz. two classes are related if they contain related elements. Let $\mathcal{P}_{0}$ be the set of (conjugacy classes of) pairs $(s, N) \in \mathcal{P}$, where $s=s(a, b), N=N_{k}$ and $(s, N)$ is not of the form in (10.13.1). By Theorem (10.12) $\mathcal{P}_{0}$ parametrises the composition factors of the standard modules $M_{s, N},(s, N) \in \mathcal{P}$. Theorems (10.5) and (10.6) may now be applied as follows.

(10.16) ThEOREM. - Let $\mathcal{P}$ and $\mathcal{P}_{0}$ be the sets of semisimple-nilpotent pairs defined in the previous paragraph, and let $\preceq$ be the partial order on $\mathcal{P}$ generated by the relation (10.15.1). Then in the Grothendieck group of finite-dimensional $\widehat{H_{n}^{a}}(q)$-modules,

$$
\left[M_{s, N}\right]=\sum_{\substack{\left(s^{\prime}, N^{\prime}\right) \in \mathcal{P}_{0} \\(s, N) \preceq\left(s^{\prime}, N^{\prime}\right)}}\left[L_{s^{\prime}, N^{\prime}}\right] \quad \text { for any pair }(s, N) \in \mathcal{P},
$$

and

$$
\left[L_{s, N}\right]=\sum_{\substack{\left(s^{\prime}, N^{\prime}\right) \in \mathcal{P} \\(s, N) \preceq\left(s^{\prime}, N^{\prime}\right)}} n_{s, N}^{s^{\prime}, N^{\prime}}\left[M_{s^{\prime}, N^{\prime}}\right]
$$

where $n_{s, N}^{s^{\prime}, N^{\prime}}=0$ or \pm 1 .

We conclude by giving some applications to the structure of the standard modules.

(10.17) COROLlary. -

(i) The standard modules $M_{s, N}((s, N) \in \mathcal{P})$ are multiplicity free.

(ii) If $q$ is not a root of unity, the standard modules have at most 2 composition factors.

(iii) In all cases, $M_{s, N}$ has composition length bounded by $[n / 2]$.

(iv) The maximum composition length of $M_{s, N}$ is $[n / 2]$, and therefore is unbounded as $n \rightarrow \infty$.

These statements are easy consequences of Theorem (10.16).

\section{Concluding remarks}

We first wish to explain how our results generalise certain aspects of the theory in [31], which involves the notion of "multisegments". Without discussing the general notion in [op. cit.], let us define these in our context as follows.

(11.1) Definition. - Let $R$ be an integral domain and $q \in R^{\times}$. A segment in $R$ is a sequence

$$
I(a, m)=\left(a, a q^{-2}, a q^{-4}, \ldots, a q^{-2(m-1)}\right)
$$

( $a \in R^{\times}, m \in \mathbb{Z}_{>0}$ ) of elements of $R^{\times}$. In addition, there is a unique segment of length 0 , which is the empty sequence. In what follows, some remarks apply only to segments of length $\neq 0$, and 
we shall rely on the context to distinguish these. Note that the elements of $I(a, m)$ are distinct if $q$ is not a root of unity. Otherwise, there may be repetitions in $I(a, m)$. Denote by $|I(a, m)|$ the multiset (i.e. set with multiplicities) underlying $I(a, m)$. Then $I(a, m)$ is determined by $|I(a, m)|$, together with its initial element.

The length of $I(a, m)$ is $m$, and we may speak of a subsegment of $I(a, m)$, with the obvious meaning, and similarly for initial and final subsegments of $I(a, m)$.

Suppose $I\left(a_{1}, m_{1}\right)$ and $I\left(a_{2}, m_{2}\right)$ are two segments. We say that $I\left(a_{1}, m_{1}\right)$ precedes $I\left(a_{2}, m_{2}\right)$ if $a_{2}=a_{1} q^{-2 i}$ for some $i \in\left\{m_{1}-m_{2}+1, m_{1}-m_{2}+2, \ldots, m_{1}\right\}$, where only the non-negative among these integers are included; i.e. if the initial element of $I\left(a_{2}, m_{2}\right)$ coincides with one of the final $m_{2}$ elements of $I\left(a_{1}, m_{1}+1\right)$. If $I\left(a_{1}, m_{1}\right)$ precedes $I\left(a_{2}, m_{2}\right)$, we may define a concatenation $I\left(a_{1}, m_{1}\right) \# I\left(a_{2}, m_{2}\right)$ as any segment which starts with $I\left(a_{1}, m_{1}+1\right)$ and finishes with $I\left(a_{2}, m_{2}\right)$. Such a segment exists by definition, and any concatenation clearly has length strictly greater than the maximum of $m_{1}$ and $m_{2}$.

Say that two segments $I\left(a_{1}, m_{1}\right)$ and $I\left(a_{2}, m_{2}\right)$ are linked if either one precedes the other. Observe that when $q$ is a root of unity, each may precede the other, and there may be many different concatenations $I\left(a_{1}, m_{1}\right) \# I\left(a_{2}, m_{2}\right)$ and $I\left(a_{2}, m_{2}\right) \# I\left(a_{1}, m_{1}\right)$

Note that in the "generic" case, when $q$ is not a root of unity, this definition (of linked segments) coincides with the definition [31, 4.1, p. 184].

We may now state our results concerning composition factors as follows. First note that the pairs $\left(s(a, b), N_{k}\right) \in \mathcal{P}$ (cf. (9.12)) correspond bijectively to pairs $I_{1}=I(a, n-k)$ and $I_{2}=I(b, k)$ of segments of length $n-k=t+k$ and $k$ respectively, where $n-k=t+k \geqslant k$. In the statement below, we write $M_{I_{1}, I_{2}}$ and $L_{I_{1}, I_{2}}$ respectively for the corresponding standard modules $M_{s(a, b), N_{k}}$ and irreducibles $L_{s(a, b), N_{k}}$ (see Section 10 above).

(11.2) THEOREM. -

(i) The standard module $M_{s, N}$ is irreducible if and only if the segments $I_{1}, I_{2}$ are not linked.

(ii) If $I_{1}$ precedes $I_{2}$, let $I_{1}^{\prime}=I_{1} \# I_{2}$ be any concatenation of $I_{1}$ and $I_{2}$. This is a segment of length $n-k^{\prime}$, where $k^{\prime}<k$ (see above). Define $I_{2}^{\prime}=I\left(b, k^{\prime}\right)$. Then the irreducible $\widehat{H_{n}^{a}}(q)$-module $L_{I_{1}^{\prime}, I_{2}^{\prime}}$ is a composition factor of $M_{I_{1}, I_{2}}$.

(iii) If $I_{2}$ precedes $I_{1}$, take $I_{1}^{\prime}$ to be any concatenation $I_{2} \# I_{1}$. This is a segment of length $n-k^{\prime}$, where again $k^{\prime}<k$. Define $I_{2}^{\prime}=I\left(a, k^{\prime}\right)$. Then the irreducible $\widehat{H_{n}^{a}}(q)$-module $L_{I_{1}^{\prime}, I_{2}^{\prime}}$ is a composition factor of $M_{I_{1}, I_{2}}$.

(iv) All composition factors of $M_{I_{1}, I_{2}}$ have multiplicity one, and are of the form $L_{I_{1}^{\prime}, I_{2}^{\prime}}$, where $\left(I_{1}^{\prime}, I_{2}^{\prime}\right)$ arises from $\left(I_{1}, I_{2}\right)$ by a sequence of transformations of the form described in (ii) and (iii) above.

(v) We have $L_{I_{1}, I_{2}}=0$ if and only if $q^{2}=-1$ and $I_{2}=-I_{1}$, so that $n=2 k$.

(vi) Say that $\left(I_{1}, I_{2}\right) \preceq\left(I_{1}^{\prime}, I_{2}^{\prime}\right)$ if $\left(I_{1}^{\prime}, I_{2}^{\prime}\right)$ is obtained from $\left(I_{1}, I_{2}\right)$ by a sequence of transformations of the form (ii) and (iii) above. Then in the Grothendieck group of finite dimensional $\widehat{H_{n}^{a}}(q)$ modules, we have

$$
\left[L_{I_{1}, I_{2}}\right]=\sum_{\left(I_{1}, I_{2}\right) \preceq\left(I_{1}^{\prime}, I_{2}^{\prime}\right)} n_{I_{1}, I_{2}}^{I_{1}^{\prime}, I_{2}^{\prime}}\left[M_{I_{1}^{\prime}, I_{2}^{\prime}}\right]
$$

where $n_{I_{1}, I_{2}}^{I_{1}^{\prime}, I_{2}^{\prime}}=0$ or \pm 1 .

These statements are simply restatements of the facts treated in (10.15) and (10.16) above.

Note the similarity between our Theorem (11.2)(i) and [31, Theorem 4.2]. The latter is of course false when $q$ is a root of unity without our modification of the meaning of linked segments. Note also that our statement holds for any $q \in \mathbb{C}$, even $q=1$. 
(11.3) We shall now give a more explicit description of the poset $\mathcal{P}$ of bisegments $P=\left(I_{1}, I_{2}\right)$, ordered by the relation $\preceq$, with a view to giving a more explicit version of Theorem (11.2)(vi). It is convenient to change notation as follows. We have defined a segment above as

$$
I(a, m)=\left(a, a q^{-2}, \ldots, f\right) \quad \text { where } f=a q^{-2(m-1)} .
$$

Write $[a, f]_{m}$ for this segment. This notation applies when $a, f \in R^{\times}$and $m$ is a positive integer such that $f=a q^{-2(m-1)}$. We call $a$ and $f$ the endpoints of the segment. The set $\mathcal{P}$ of bisegments is parametrised by unordered pairs $P=\left\{[a, f]_{l},[b, q]_{k}\right\}$ of segments such that $k+l=n$. We say $P$ has length $n=k+l$ and write $k(P)=\min (k, l)$. The corresponding standard module and irreducible modules will be denoted $M_{P}, L_{P}$ respectively.

Suppose $P$ and $Q$ are bisegments of the same length. We say that $P$ and $Q$ are transverse if they are of the form $\left\{[a, f]_{l},[b, g]_{k}\right\}$ and $\left\{[a, g]_{i},[b, f]_{j}\right\}$ respectively; we say $P$ and $Q$ are parallel if they are of the form $\left\{[a, f]_{l},[b, g]_{k}\right\}$ and $\left\{[a, f]_{i},[b, g]_{j}\right\}$ respectively. (Note that the subscripts may be zero.)

Given a pair $Q, Q^{\prime}$ of parallel bisegments of the same length, we say that $Q$ and $Q^{\prime}$ have the same orientation if they are of the form $\left\{[a, f]_{l},[b, g]_{k}\right\}$ and $\left\{[a, f]_{i},[b, g]_{j}\right\}$ respectively with $k \leqslant l$ and $j \leqslant i$; otherwise $Q$ and $Q^{\prime}$ have opposite orientations.

The following assertions concerning the combinatorics of the poset $\mathcal{P}$ may be easily checked.

(i) If $P, P^{\prime} \in \mathcal{P}$ and $P \preceq P^{\prime}$, then $P^{\prime}$ is either transverse or parallel to $P$ (possibly both).

(ii) If $P^{\prime}$ is transverse to $P$ and $k\left(P^{\prime}\right)<k(P)$, then $P \stackrel{\circ}{\prec} P^{\prime}$.

(iii) If $P$ is transverse to itself, then $\left\{P^{\prime} \in \mathcal{P} \mid P \preceq P^{\prime}\right\}$ is totally ordered. If $P_{1}$ is the minimal element of $\left\{P^{\prime} \in \mathcal{P} \mid P \prec P^{\prime}\right\}$ (i.e. $P_{1} \neq P$ ) then

$$
\left[L_{P}\right]=\left[M_{P}\right]-\left[M_{P_{1}}\right] .
$$

(iv) If $P, P^{\prime}$ are parallel, have the same orientation, $k\left(P^{\prime}\right)<k(P)$, and there exists $Q$ with $P \prec Q$, then there is a bisegment $P_{1} \in \mathcal{P}$ such that $P \stackrel{\circ}{\prec} P_{1} \stackrel{\circ}{\prec} P^{\prime}$.

(v) Say that $P \neq P^{\prime} \in \mathcal{P}$ are opposed if they are parallel and not comparable. Then given $Q \in \mathcal{P}$ such that there exists $P \in \mathcal{P}$ with $Q \prec P$, there exists at most one element $Q^{\prime} \in \mathcal{P}$ such that $Q, Q^{\prime}$ are opposed.

The proof of (v) proceeds by showing that if $Q$ is opposed to both $Q^{\prime}$ and $Q^{\prime \prime}$ then $Q^{\prime}, Q^{\prime \prime}$ are opposed to each other, and hence by (iv) they must have opposite orientations. But they both have orientation opposite to that of $Q$, whence two such elements $Q^{\prime}, Q^{\prime \prime}$ cannot exist.

(vi) Suppose that $P_{1}$ is not transverse to itself. If $P_{1} \prec Q \prec P_{2}$, then there exists a unique bisegment $Q^{\prime}$ which is opposed to $Q$.

(vii) If $P \prec Q$, then the number of bisegments $R$ in the interval $[P, Q]:=\{R \mid P \preceq R \preceq Q\}$ which are parallel to $P$ is equal to the number of $R$ which are transverse to $P$.

The structure of the poset $\mathcal{P}$ is completely determined by the properties (i) to (vii) above, which are all straightforward to prove. Moreover it is an easy consequence of (vii) that

(11.4) Proposition. - Suppose $P \in \mathcal{P}$ is not transverse to itself. Then

$$
\left[L_{P}\right]=\sum_{P \preceq Q} n_{P}^{Q}\left[M_{Q}\right]
$$

where

$$
n_{P}^{Q}= \begin{cases}1 & \text { if } Q \text { is parallel to } P \\ -1 & \text { if } Q \text { is transverse to } P .\end{cases}
$$


We remark also that given the results of $[20,1,10]$, our statement (11.2)(vi) implies that the Kazhdan-Lusztig polynomials which arise from intersection complexes on the closure of the $Z_{G}(s)$ orbit of the nilpotent element $N$ are equal to 1 .

Finally, we observe that the results of this paper may be used to discuss aspects of the representation theory of the affine Hecke algebra $\widehat{H_{n}^{a}}(q)$ over any algebraically closed field of positive characteristic, i.e. the "modular case". This is carried out for the algebras $T L B_{n}(q, Q)$ in [6].

\section{Acknowledgement}

Both authors thank the Australian Research Council for support. The second author thanks the Alexander von Humboldt Stiftung for support and the Universität Bielefeld for hospitality during the preparation of this work.

\section{REFERENCES}

[1] ARIKI S., On the decomposition numbers of the Hecke algebra of $G(m, 1, n)$, J. Math. Kyoto Univ. 36 (4) (1996) 789-808.

[2] Ariki S., Mathas A., The number of simple modules of the Hecke algebras of type $G(r, 1, n)$, Math. Z., to appear.

[3] Benson D.J., Representations and Cohomology. I. Basic Representation Theory of Finite Groups and Associative Algebras, Second Edition, in: Cambridge Stud. Adv. Math., Vol. 30, Cambridge University Press, Cambridge, 1998.

[4] Bernstein I.N., Zelevinsky A.V., Induced representations of reductive p-adic groups, Ann. Sci. Éc. Norm. Sup. (4) 10 (1977) 441-472.

[5] Chriss N., Ginzburg V., Representation Theory and Complex Geometry, Birkhäuser Boston, Boston, MA, 1997.

[6] Cox A., Graham J.J., Martin P., The blob algebra in positive characteristic, Preprint, 2001.

[7] Deligne P., Les immeubles des groupes de tresses généralisés, Invent. Math. 17 (1972) 273-302.

[8] FAN C.K., GREEN R.M., On the affine Temperley-Lieb algebras, J. London Math. Soc. (2) 60 (1999) 366-380.

[9] GinZBURG V., Lagrangian construction of representations of Hecke algebras, Adv. in Math. 63 (1987) $100-112$.

[10] GROJNOWSKI I., Representations of affine Hecke algebras (and affine quantum $G L_{n}$ ) at roots of unity, Internat. Math. Res. Notices 5 (1994).

[11] GREEN R.M., On representations of affine Temperley-Lieb algebras, in: Algebras and Modules II, in: CMS Conference Proceedings, Vol. 24, American Math. Society, Providence, RI, 1998, pp. 245261.

[12] Geck M., LAmbropoulou S., Markov traces and knot invariants related to Iwahori-Hecke algebras of type B, J. Reine Angew. Math. 482 (1997) 191-213.

[13] Graham J.J., Lehrer G.I., Cellular algebras, Invent. Math. 123 (1996) 1-34.

[14] GRAhAm J.J., LehreR G.I., The representation theory of affine Temperley-Lieb algebras, Enseign. Math. 44 (1998).

[15] Graham J.J., LehreR G.I., The two-step nilpotent representations of the extended affine Hecke algebra of type A, Compositio Math. 133 (2002) 173-197.

[16] JONES V.F.R., A quotient of the affine Hecke algebra in the Brauer algebra, Enseign. Math. 40 (1994) 313-344.

[17] JONES V.F.R., Hecke algebra representations of braid groups and link polynomials, Ann. of Math. 126 (1987) 335-388.

[18] Jones V.F.R., Planar algebras, Preprint on server, 2001.

[19] KaZhdan D., Lusztig G., Proof of the Deligne-Langlands conjecture for Hecke algebras, Invent. Math. 87 (1987) 153-215. 
[20] Lascoux A., Leclerc B., Thibon J.-Y., Hecke algebras at roots of unity and crystal bases of quantum affine algebras, Comm. Math. Phys. 181 (1996) 205-263.

[21] Leclerc B., Thibon J.-Y., VAsserot E., Zelevinsky's involution at roots of unity, J. Reine Angew. Math. 513 (1999) 33-51.

[22] LuszTig G., Some examples of square integrable representations of semisimple $p$-adic groups, Trans. Amer. Math. Soc. 277 (1983) 623-653.

[23] Lusztig G., Notes on affine Hecke algebras, Preprint, MIT, 2000.

[24] Lusztig G., Affine Hecke algebras and their graded version, J. Amer. Math. Soc. 2 (1989) 599-635.

[25] Martin P.P., Woodcock D., On the structure of the blob algebra, J. Algebra 225 (2000) 957-988.

[26] Martin P., Saleur H., The blob algebra and the periodic Temperley-Lieb algebra, Lett. Math. Phys. 30 (1994) 189-206.

[27] NGUYEN V.D., The fundamental groups of the spaces of regular orbits of the affine Weyl groups, Topology 22 (1983) 425-435.

[28] Rogawski J.D., On modules over the Hecke algebra of a $p$-adic group, Invent. Math. 79 (1985) 443-465.

[29] том DiEcK T., Symmetrische Brücken und Knotentheorie zu den Dynkin-Diagrammen vom Typ $B$, J. Reine Angew. Math. 451 (1994) 71-88.

[30] XI N., Representations of Affine Hecke Algebras, in: Lecture Notes in Math., Vol. 1587, SpringerVerlag, Berlin, 1994.

[31] ZELEVINSKY A.V., Induced representations of reductive $p$-adic groups. II. On irreducible representations of GL(n), Ann. Sci. Éc. Norm. Sup. 13 (1980) 165-210.

[32] Zelevinsky A.V., The p-adic analogue of the Kazhdan-Lusztig conjecture, Funktsional. Anal. $i$ Prilozhen. 15 (1981) 9-21 (Russian).

J.J. GRAHAM

School of Mathematics and Statistics,

The University of Birmingham,

Birmingham B15 2TT, UK

G.I. LEHRER

School of Mathematics and Statistics,

University of Sydney,

Sydney, N.S.W. 2006, Australia

E-mail: gusl@maths.usyd.edu.au 